Supporting Information for

\title{
Degradation of Polydienes Induced by Alkyllithium: Characterization and Reaction Mechanism
}

Jian Tang ${ }^{1}$, Tinghao Xie ${ }^{1}$, Yuka Yuan ${ }^{1}$, Jing Hua ${ }^{1}$, Tao Zhuang ${ }^{1}$, Yi Luo ${ }^{2}$, Jieting Geng ${ }^{1}$

${ }^{1}$ Key Laboratory of Rubber-Plastics, Ministry of Education / Shandong Provincial Key

Laboratory of Rubber-plastics, Qingdao University of Science and Technology, Qingdao 266042, P.R. China.

${ }^{2}$ Dalian University of Technology, School of Chemical Engineering, Liaoning 116024, P.R. China.

Email correspondence to: huajing72@qust.edu.cn 


\section{Table of contents}

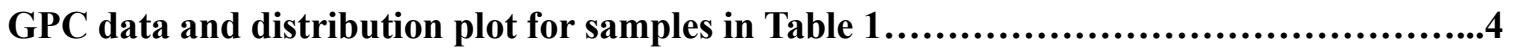

Figure S1. GPC data and distribution plot of cis-PB (Table 1. Entry 1) .......................

Figure S2. GPC data and distribution plot of blank control for cis-PB ....................... 4

Figure S3. GPC data and distribution plot of cis-PB degraded PB (Table 1. Entry 2) ..............5

Figure S4. GPC data and distribution plot of cis-PB degraded PB (Table 1. Entry 3) ..............5

Figure S5. GPC data and distribution plot of cis-PB degraded PB (Table 1. Entry 4) ..............6

Figure S6. GPC data and distribution plot of cis-PB degraded PB (Table 1. Entry 5) ..............6

Figure S7. GPC data and distribution plot of cis-PB degraded PB (Table 1. Entry 6) ...............7

Figure S8. GPC data and distribution plot of cis-PB degraded PB (Table 1. Entry 7) ..............

Figure S9. GPC data and distribution plot of cis-PB degraded PB (Table 1. Entry 8) .............8

Figure S10. GPC data and distribution plot of cis-PB degraded PB (Table 1. Entry 9) ............8

Figure S11. GPC data and distribution plot of cis-PB degraded PB (Table 1. Entry 10) ............

Figure S12. GPC data and distribution plot of cis-PB degraded PB (Table 1. Entry 11) ............9

Figure S13. GPC data and distribution plot of cis-PB degraded PB (Table 1. Entry 12) ..........10

Figure S14. GPC data and distribution plot of cis-PB degraded PB (Table 1. Entry 13) ..........10

Figure S15. GPC data and distribution plot of cis-PB degraded PB (Table 1. Entry 14) ...........11

Figure S16. GPC data and distribution plot of cis-PB degraded PB (Table 1. Entry 15) ..........11

Figure S17. GPC data and distribution plot of cis-PB degraded PB (Table 1. Entry 16) ...........12

Figure S18. GPC data and distribution plot of cis-PB degraded PB (Table 1. Entry 17) ..........12

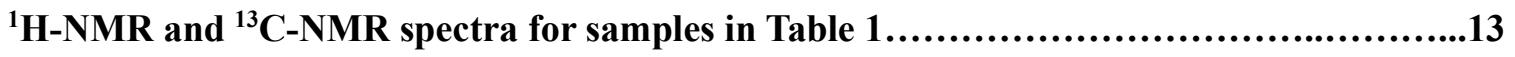

Figure S19. ${ }^{1} \mathrm{H}-\mathrm{NMR}$ spectrum of blank control for cis-PB............................... 13

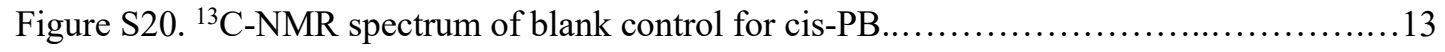

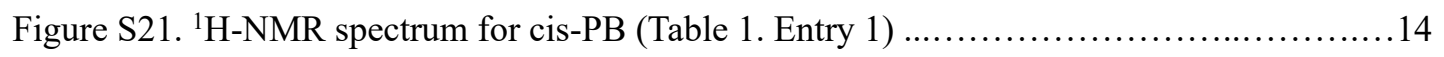

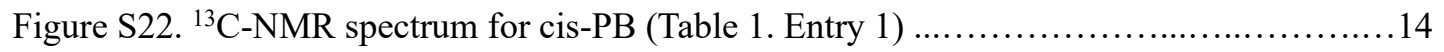

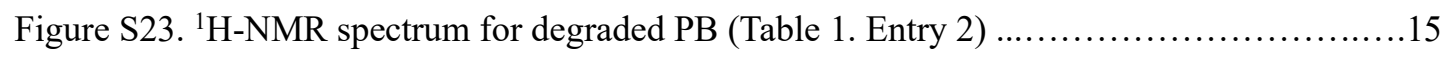

Figure S24. ${ }^{13} \mathrm{C}-\mathrm{NMR}$ spectrum for cis-PB (Table 1. Entry 2) ............................... 15

Figure S25. ${ }^{1} \mathrm{H}-\mathrm{NMR}$ spectrum for degraded PB (Table 1. Entry 3) ............................. 16

Figure S26. ${ }^{13} \mathrm{C}-\mathrm{NMR}$ spectrum for cis-PB (Table 1. Entry 3 ) .............................. 16

Figure S27. ${ }^{1} \mathrm{H}-\mathrm{NMR}$ spectrum for degraded PB (Table 1. Entry 4) ......................... 17

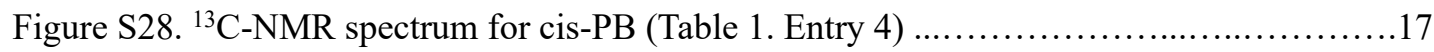

Figure S29. ${ }^{1} \mathrm{H}-\mathrm{NMR}$ spectrum for degraded PB (Table 1. Entry 5) .............................

Figure S30. ${ }^{13} \mathrm{C}-\mathrm{NMR}$ spectrum for cis-PB (Table 1. Entry 5) ................................ 18

Figure S31. ${ }^{1} \mathrm{H}-\mathrm{NMR}$ spectrum for degraded PB (Table 1. Entry 6) ............................

Figure S32. ${ }^{13} \mathrm{C}-\mathrm{NMR}$ spectrum for cis-PB (Table 1. Entry 6) .................................

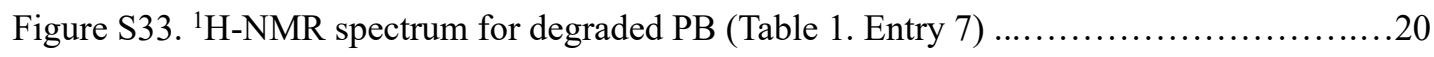

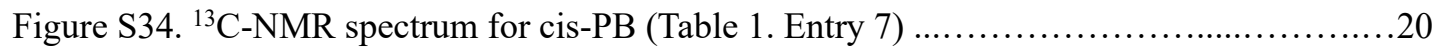

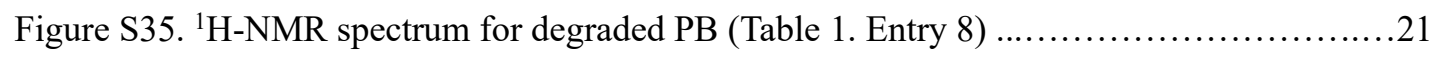

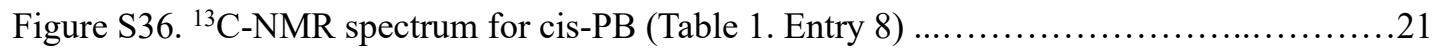




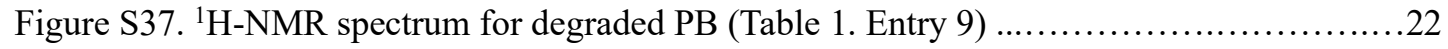

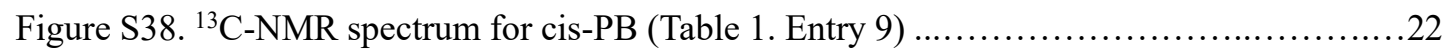

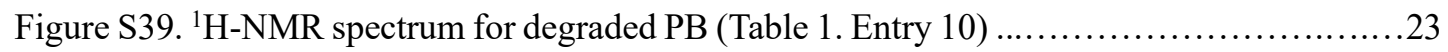

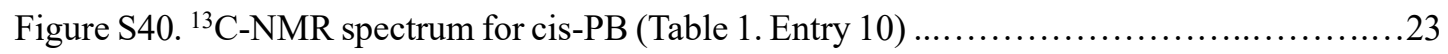

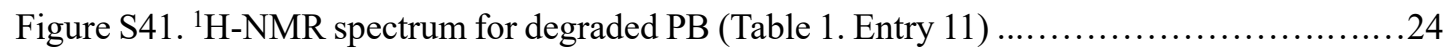

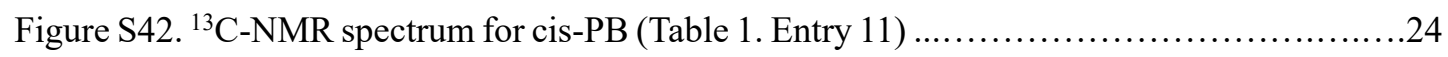

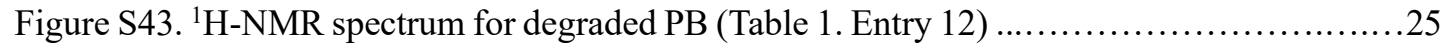

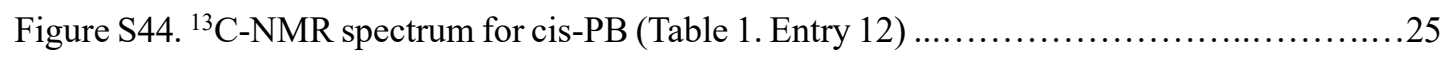

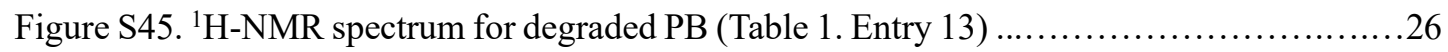

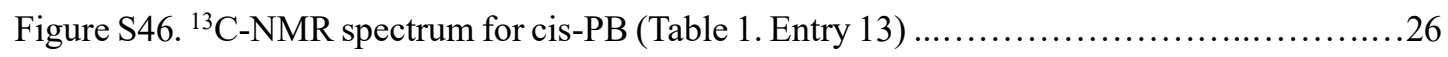

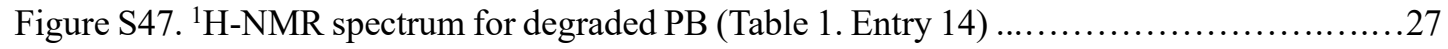

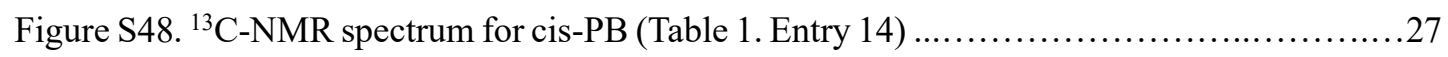

Figure S49. ${ }^{1} \mathrm{H}-\mathrm{NMR}$ spectrum for degraded PB (Table 1. Entry 15) ......................... 28

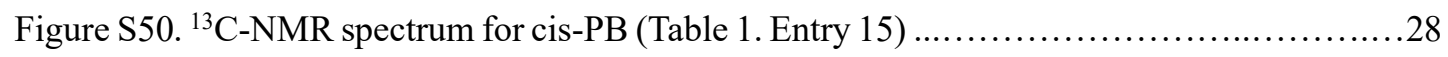

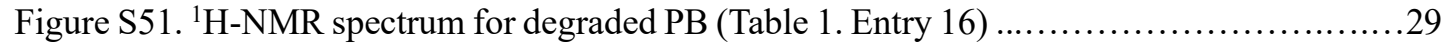

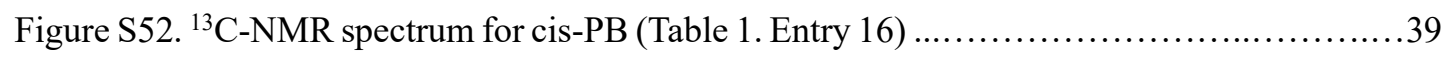

Figure S53. ${ }^{1} \mathrm{H}-\mathrm{NMR}$ spectrum for degraded PB (Table 1. Entry 17) ......................... 30

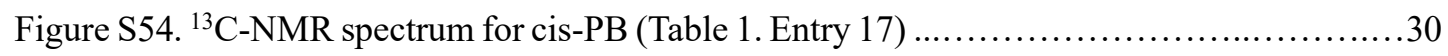

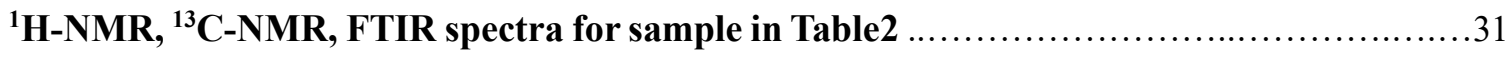

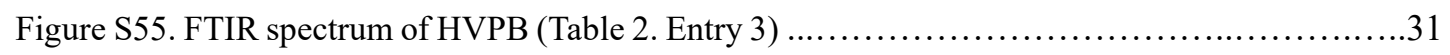

Figure S56. FTIR spectrum of HVPB (Table 2. Entry 4) .........................................

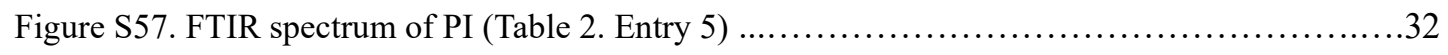

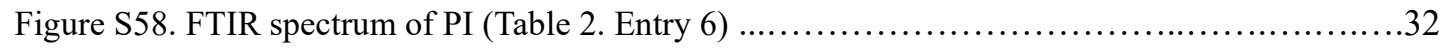

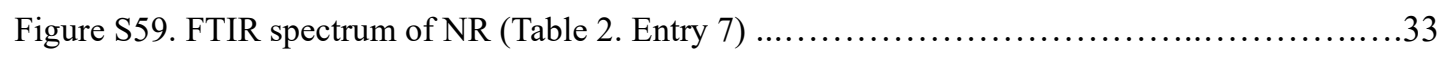

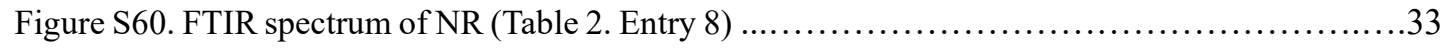

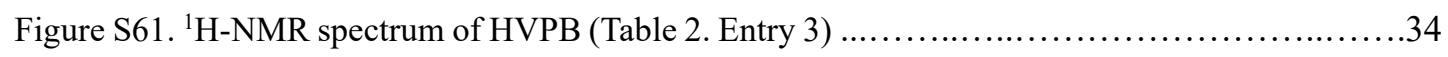

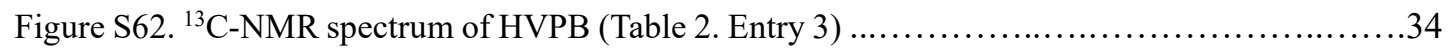

Figure S63. 1H-NMR spectrum of HVPB after degradation (Table 2. Entry 4) ...................... 35

Figure S64. ${ }^{13} \mathrm{C}-\mathrm{NMR}$ spectrum of HVPB after degradation (Table 2. Entry 4) .................... 35

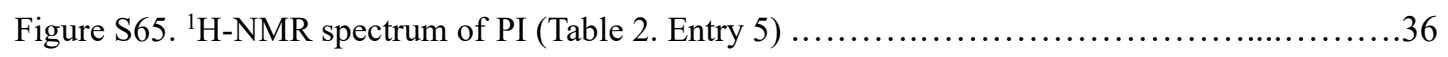

Figure S66. ${ }^{13} \mathrm{C}$-NMR spectrum of PI (Table 2. Entry 5) ........................................ 36

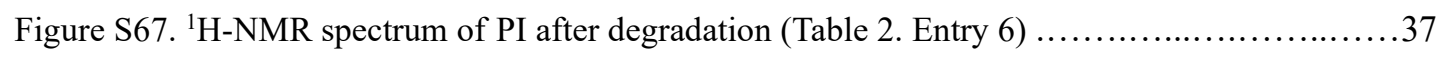

Figure S68. ${ }^{13} \mathrm{C}-\mathrm{NMR}$ spectrum of PI after degradation (Table 2. Entry 6) ........................ 37

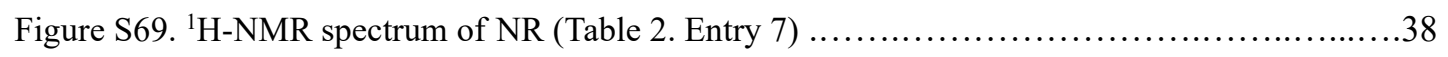

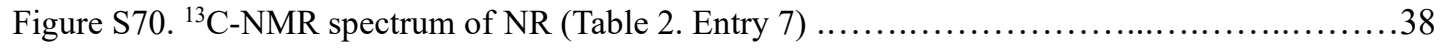

Figure S71. ${ }^{1} \mathrm{H}-\mathrm{NMR}$ spectrum of NR after degradation (Table 2. Entry 8) ........................ 39

Figure S72. ${ }^{13} \mathrm{C}$-NMR spectrum of NR after degradation (Table 2. Entry 8) ....................... 39

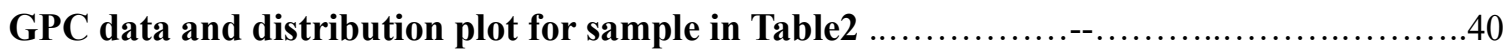

2D HSQC-NMR spectrum of squalene degradation products (Figure S81) ....................44

Cartesian coordinates of all optimized structure for DFT calculations.....................45 


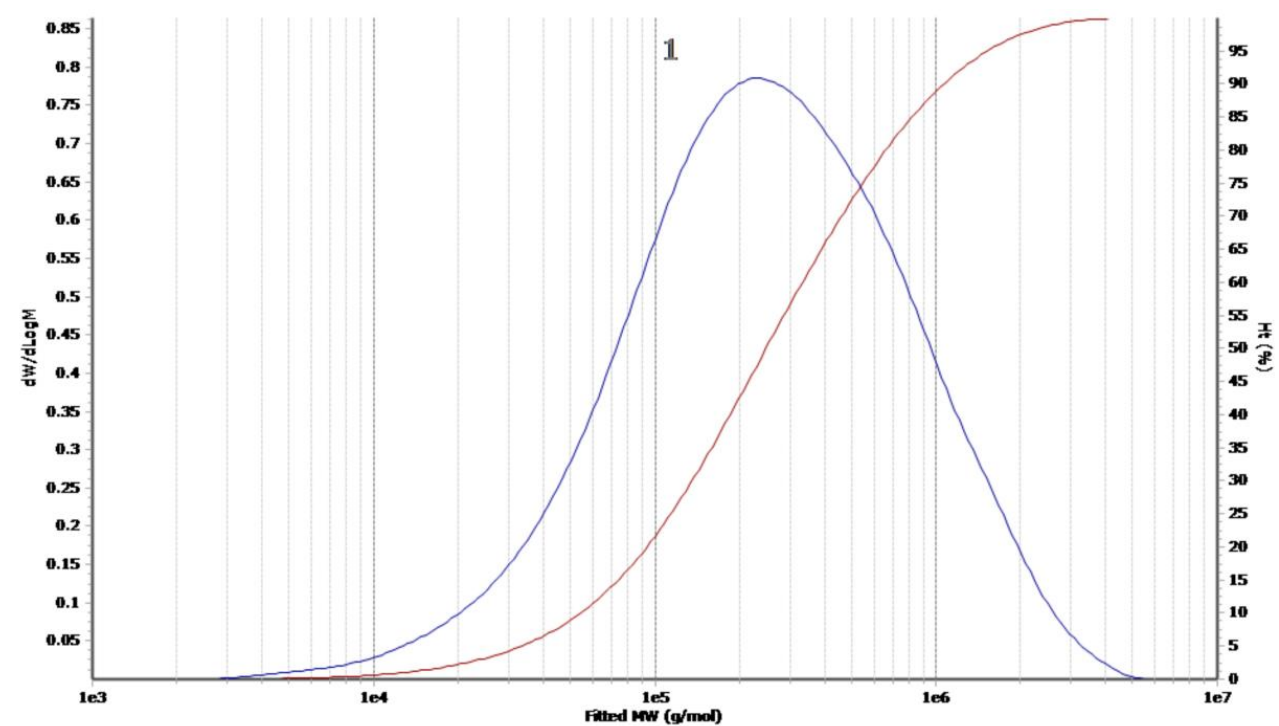

Molecular Weight Averages
\begin{tabular}{|c|c|c|c|c|c|r|r|}
\hline Peak & Mp & Mn & Mw & Mz & Mz+1 & Mv & PD \\
\hline Peak 1 & 233772 & 115696 & 438819 & 1084159 & 1836562 & 981390 & 3.793 \\
\hline
\end{tabular}

Figure S1.GPC data and distribution plot of cis-PB (Table 1. Entry 1)

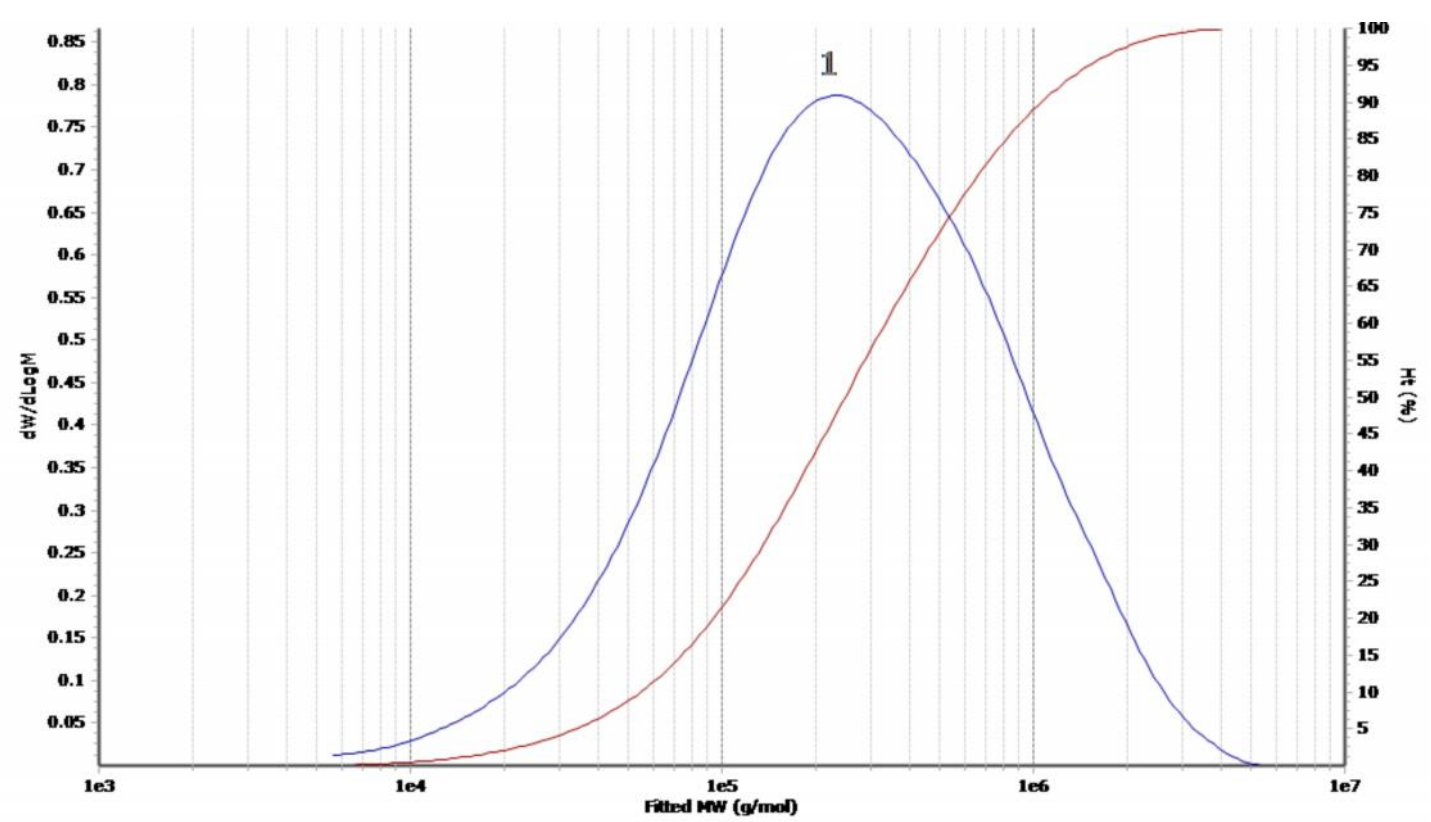

Molecular Weight Averages

\begin{tabular}{|c|c|c|c|c|c|r|r|}
\hline Peak & Mp & Mn & Mw & Mz & Mz+1 & Mv & PD \\
\hline Peak 1 & 238273 & 120803 & 438103 & 1075676 & 1812367 & 974506 & 3.627 \\
\hline
\end{tabular}

Figure S2.GPC data and distribution plot of blank control for cis-PB (reaction condition: in $\mathrm{n}$-hexane, $80^{\circ} \mathrm{C}, 240 \mathrm{~min}$, no alkyllithium/TMEDA added) 


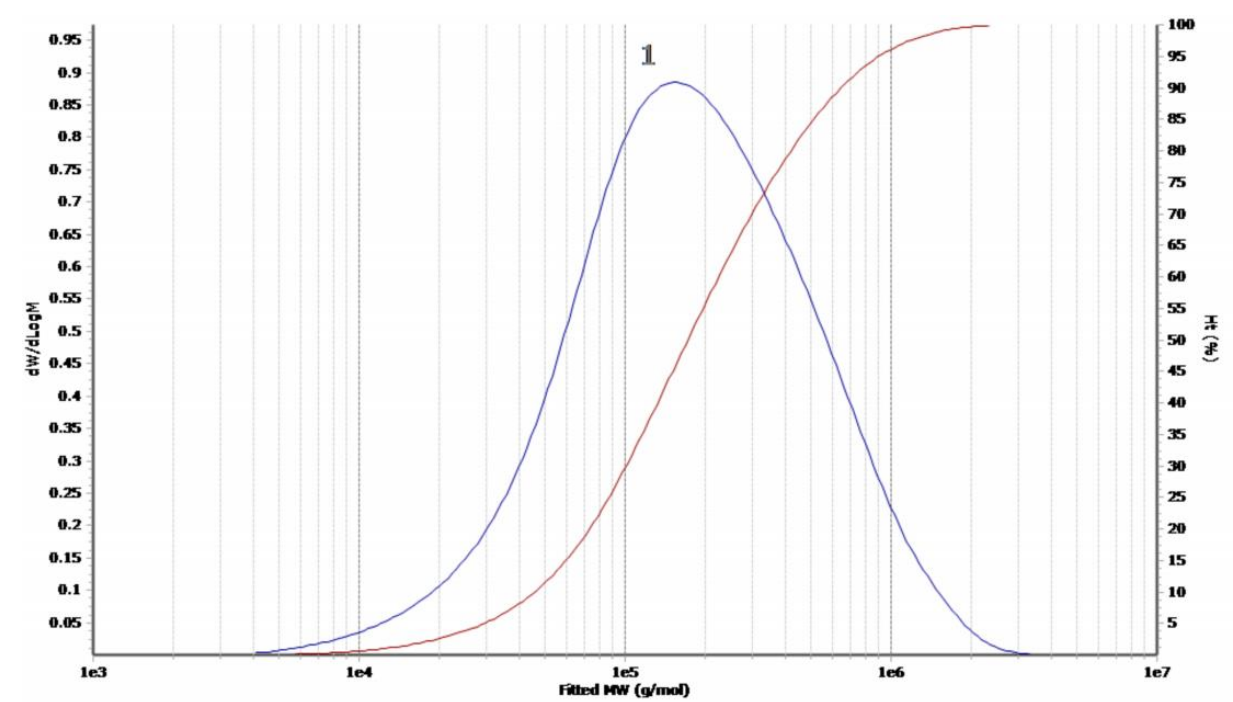

Molecular Weight Averages

\begin{tabular}{|c|c|c|r|r|r|r|r|}
\hline Peak & Mp & Mn & Mw & Mz & Mz+1 & Mv & PD \\
\hline Peak 1 & 154298 & 96206 & 279451 & 623362 & 1043688 & 567111 & 2.905 \\
\hline
\end{tabular}

Figure S3.GPC data and distribution plot of cis-PB degraded PB (Table 1. Entry 2)

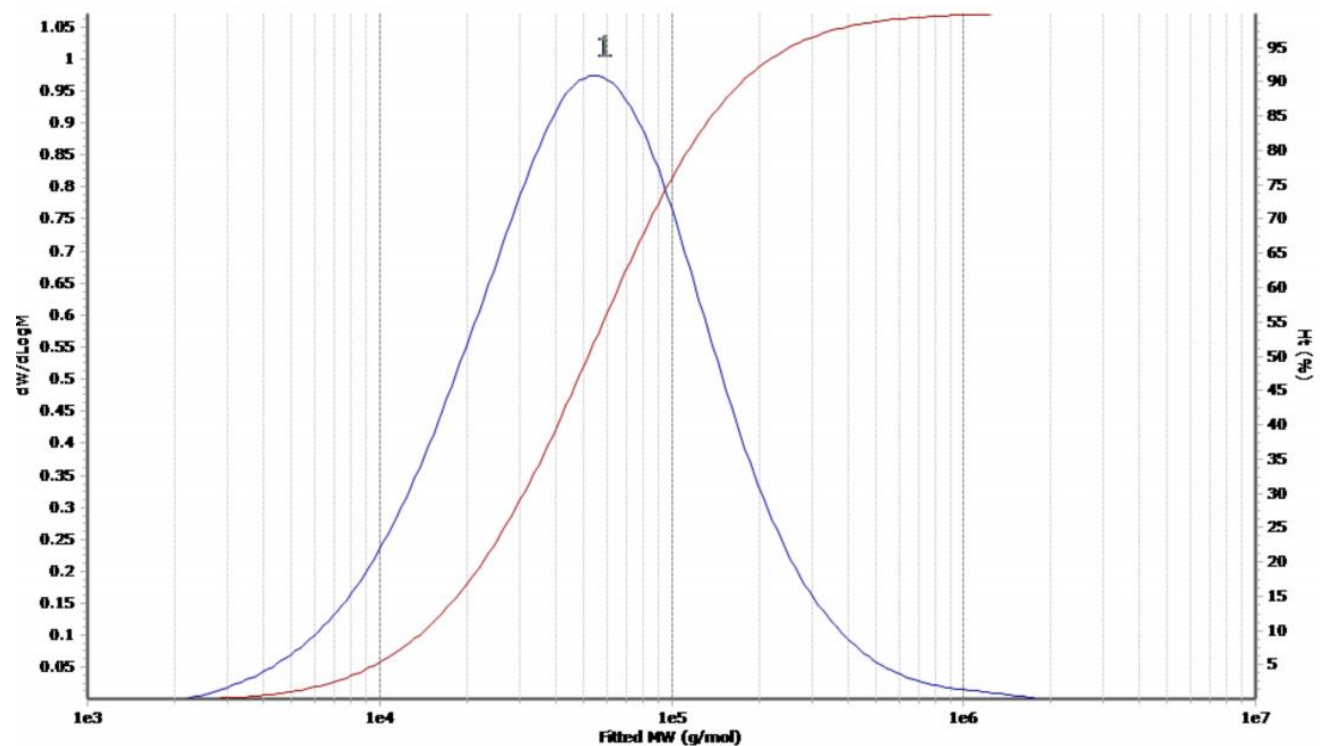

Molecular Weight Averages

\begin{tabular}{|c|c|c|c|c|c|r|r|}
\hline Peak & Mp & Mn & Mw & Mz & Mz+1 & Mv & PD \\
\hline Peak 1 & 54120 & 31323 & 83135 & 229662 & 556705 & 197194 & 2.654 \\
\hline
\end{tabular}

Figure S4.GPC data and distribution plot of cis-PB degraded PB (Table 1. Entry 3) 


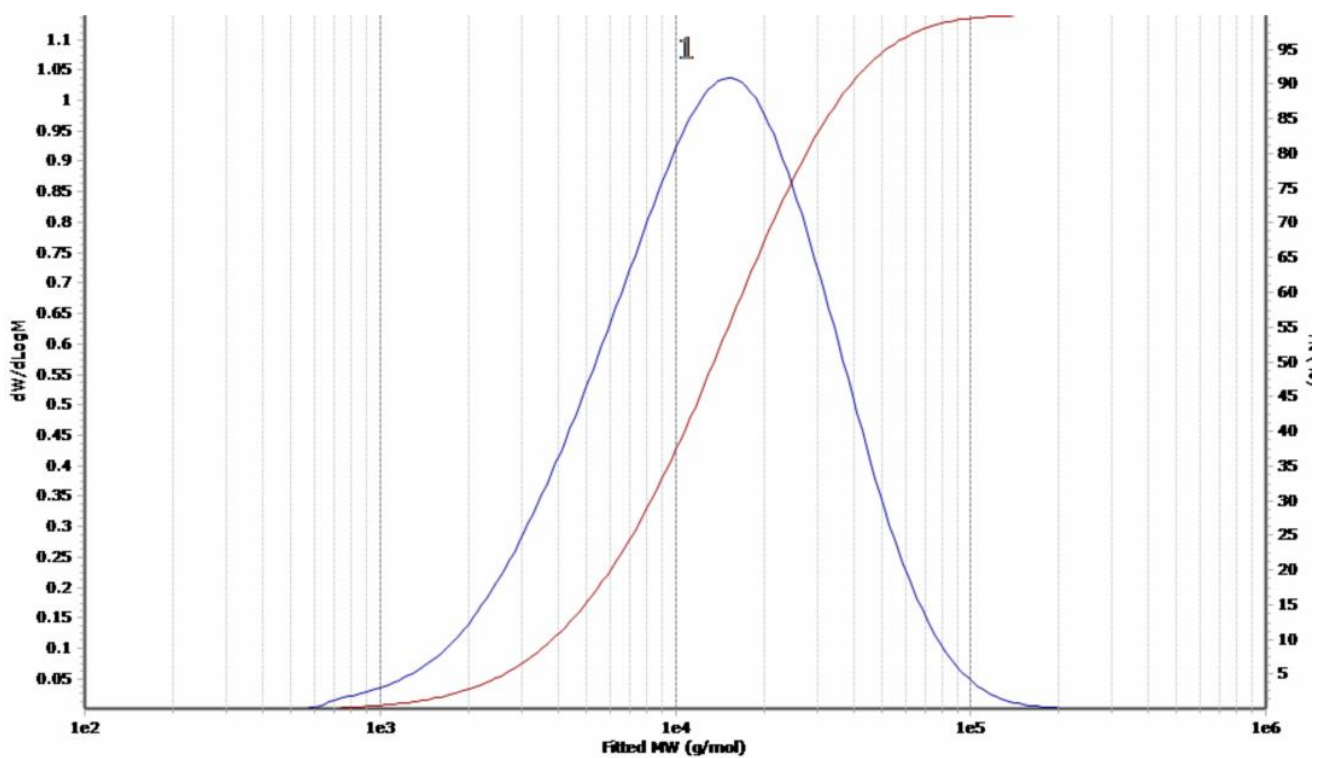

\section{Molecular Weight Averages}

Molecular Weight Averages
\begin{tabular}{|c|c|c|c|r|r|r|r|}
\hline Peak & Mp & Mn & Mw & Mz & \multicolumn{1}{|c|}{ Mz+1 } & Mv & PD \\
\hline Peak 1 & 15283 & 8270 & 18562 & 34554 & 55879 & 31900 & 2.244 \\
\hline
\end{tabular}

Figure S5.GPC data and distribution plot of cis-PB degraded PB (Table 1. Entry 4)

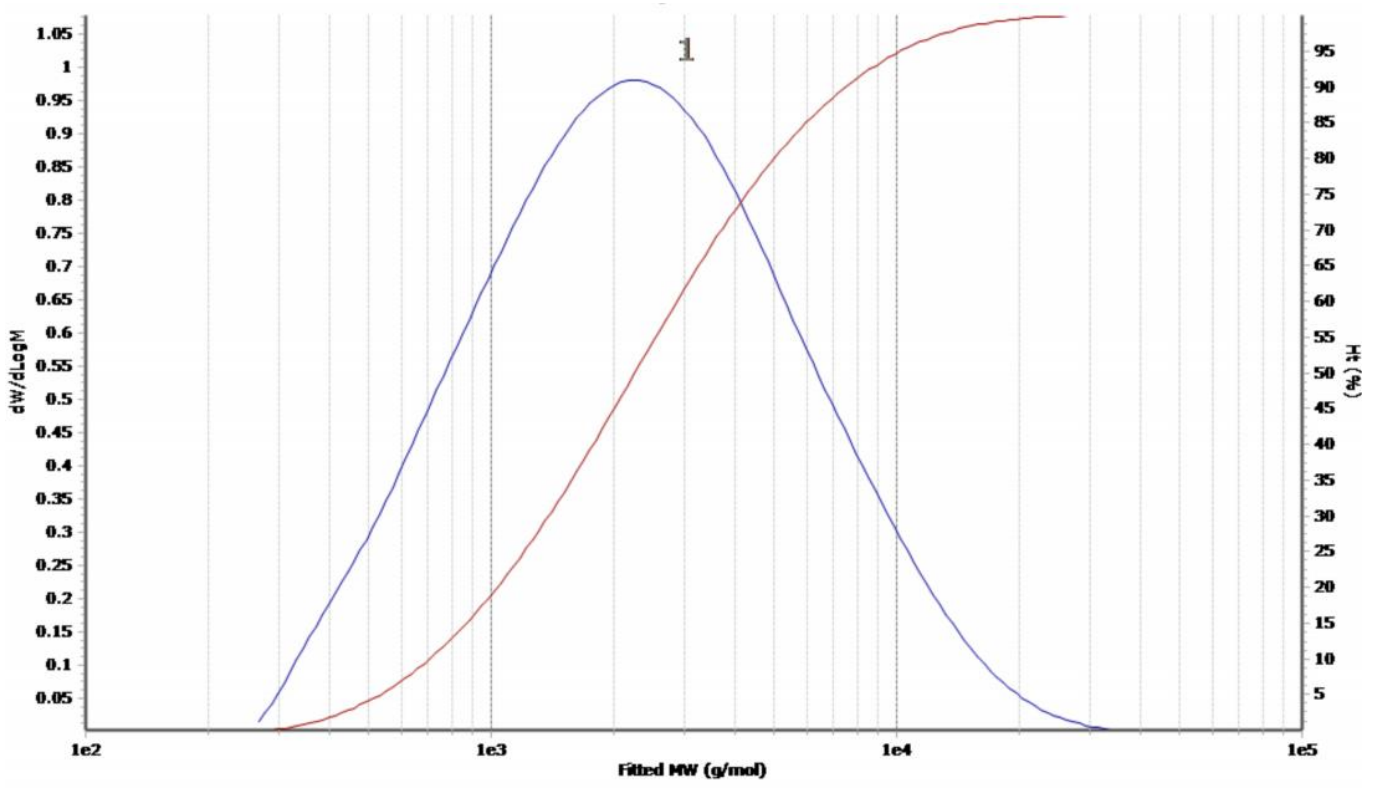

Figure S6.GPC data and distribution plot of cis-PB degraded PB (Table 1. Entry 5) 


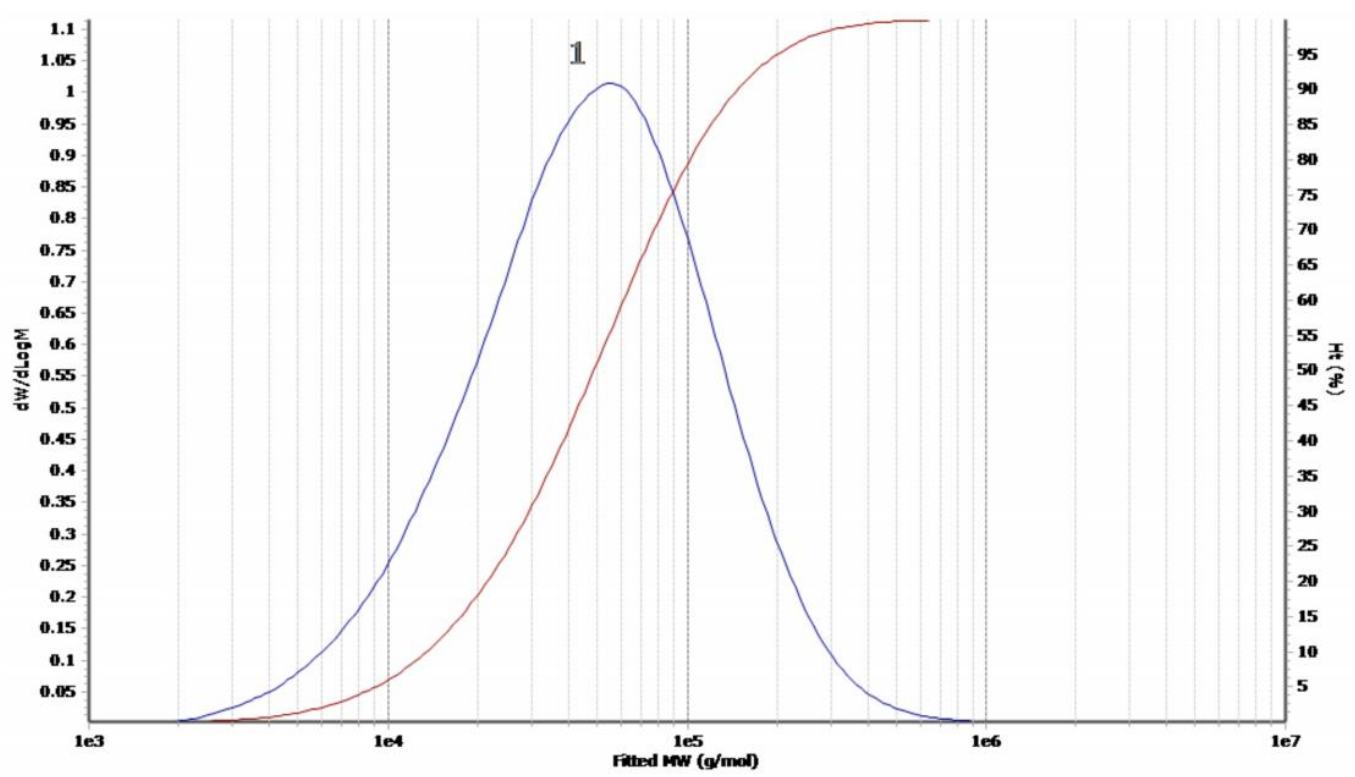

Molecular Weight Averages

\begin{tabular}{|c|c|r|r|r|r|r|r|}
\hline Peak & Mp & Mn & Mw & Mz & Mz+1 & Mv & PD \\
\hline Peak 1 & 56111 & 29293 & 69415 & 141549 & 258380 & 128429 & 2.37 \\
\hline
\end{tabular}

Figure S7.GPC data and distribution plot of cis-PB degraded PB (Table 1. Entry 6)

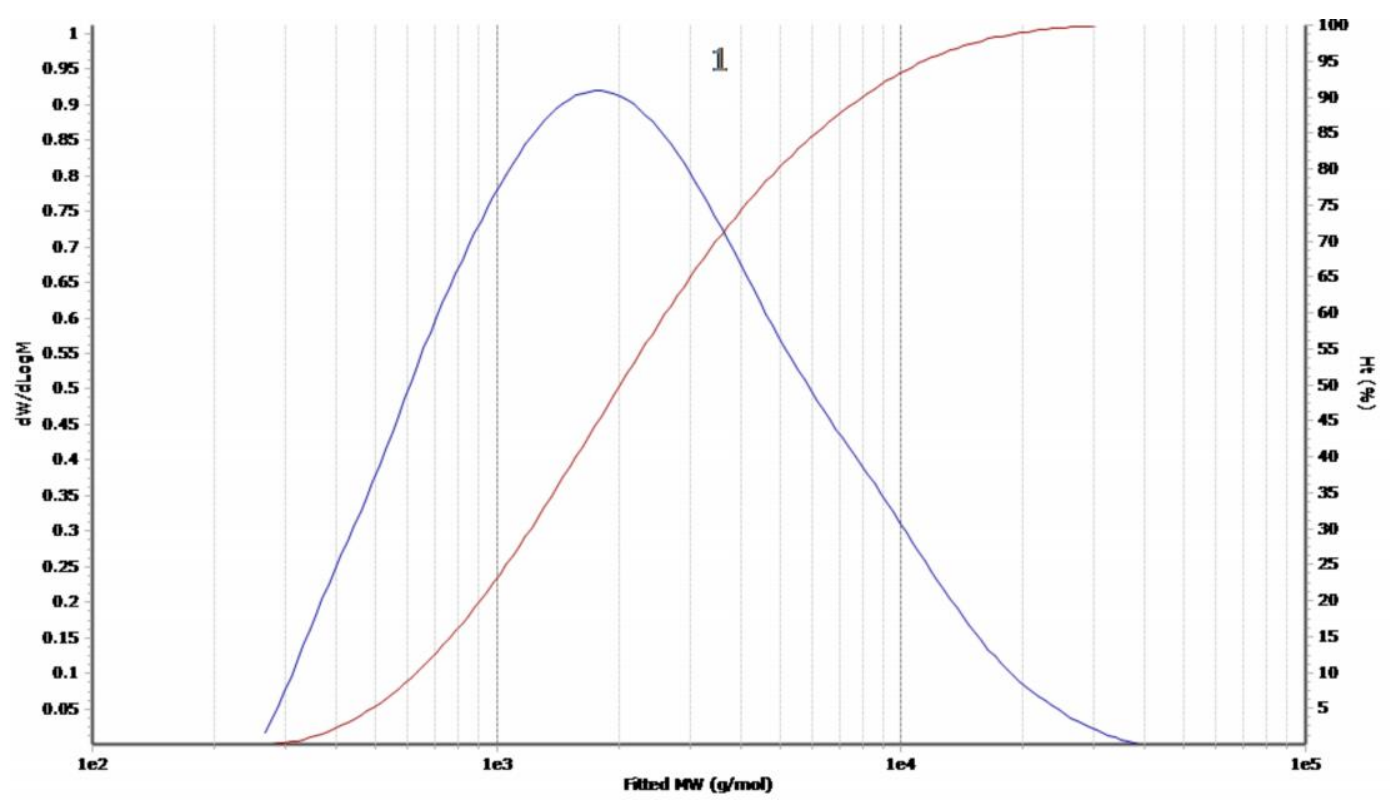

Figure S8.GPC data and distribution plot of cis-PB degraded PB (Table 1. Entry 7)

Molecular Weight Averages

\begin{tabular}{|c|c|r|r|r|r|r|r|}
\hline Peak & Mp & Mn & Mw & Mz & Mz+1 & Mv & \multicolumn{1}{c|}{ PD } \\
\hline Peak 1 & 1781 & 1433 & 3421 & 7963 & 13725 & 7188 & 2.387 \\
\hline
\end{tabular}




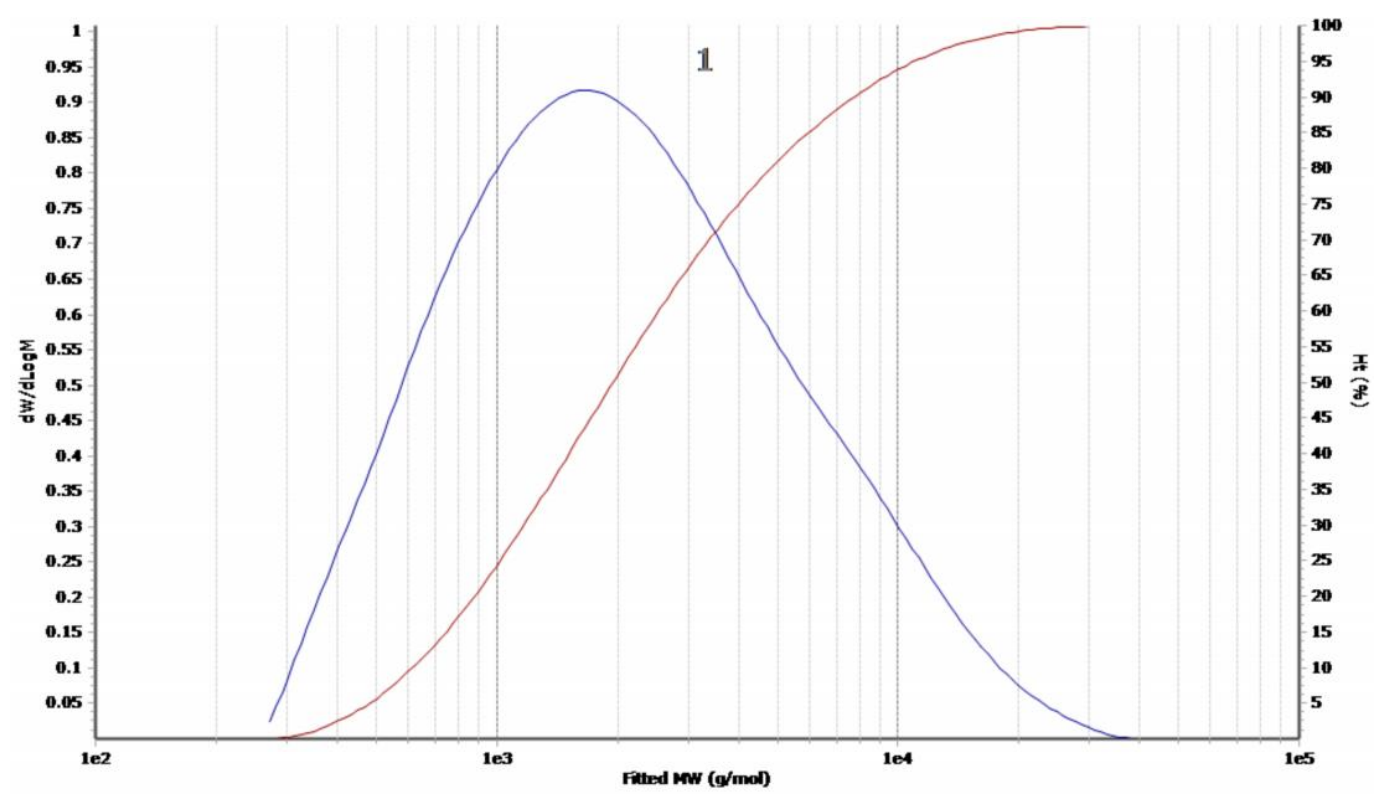

Molecular Weight Averages

\begin{tabular}{|c|r|r|r|r|r|r|r|}
\hline Peak & Mp & Mn & Mw & \multicolumn{1}{|c|}{ Mz } & Mz+1 & Mv & PD \\
\hline Peak 1 & 1657 & 1393 & 3304 & 7621 & 13009 & 6892 & 2.372 \\
\hline
\end{tabular}

Figure S9.GPC data and distribution plot of cis-PB degraded PB (Table 1. Entry 8)

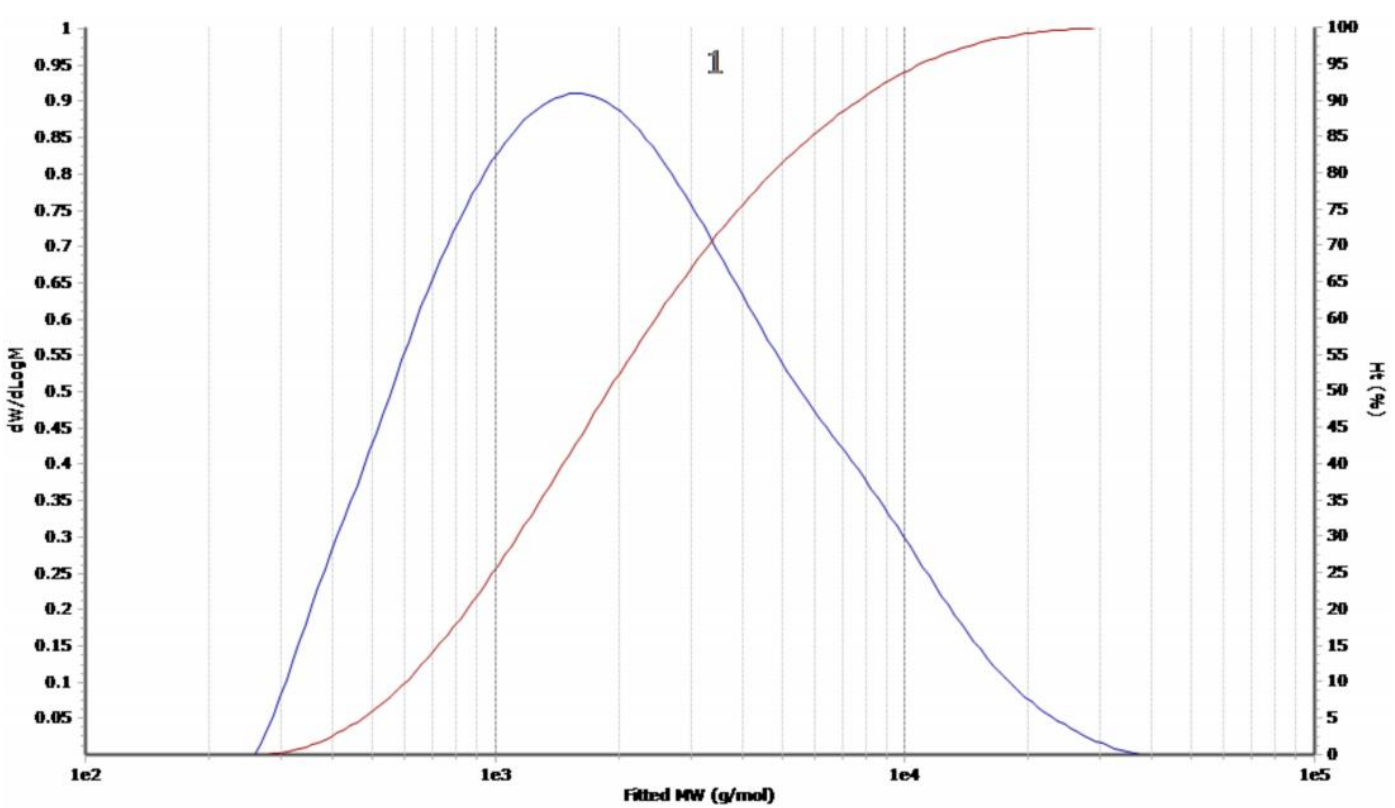

Molecular Weight Averages

\begin{tabular}{|c|r|r|r|r|r|r|r|}
\hline Peak & Mp & \multicolumn{1}{c|}{ Mn } & Mw & Mz & Mz+1 & Mv & PD \\
\hline Peak 1 & 1570 & 1361 & 3263 & 7663 & 13133 & 6920 & 2.398 \\
\hline
\end{tabular}

Figure S10.GPC data and distribution plot of cis-PB degraded PB (Table 1. Entry 9) 


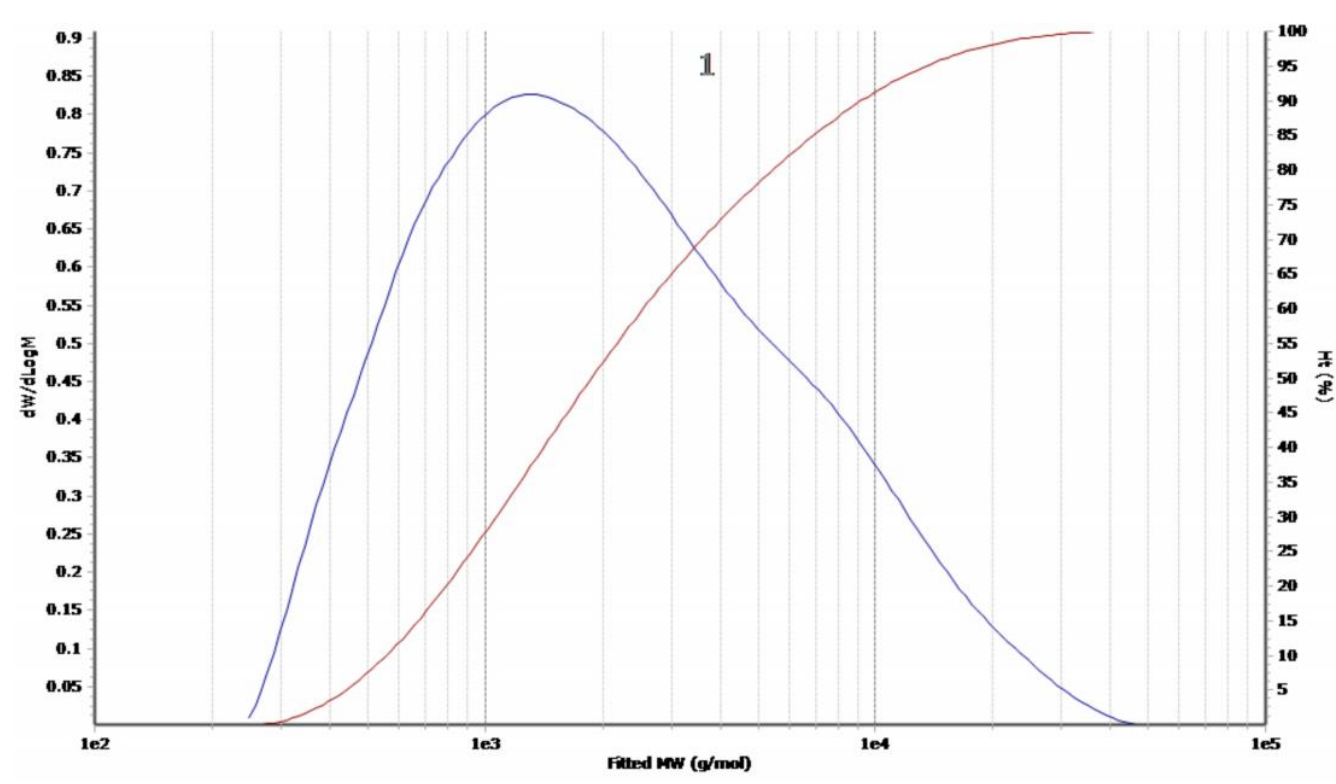

Molecular Weight Averages

\begin{tabular}{|c|c|r|r|r|r|r|r|}
\hline Peak & Mp & Mn & Mw & \multicolumn{1}{|c|}{ Mz } & \multicolumn{1}{|c|}{ Mz+1 } & Mv & PD \\
\hline Peak 1 & 1310 & 1307 & 3706 & 9900 & 17123 & 8885 & 2.836 \\
\hline
\end{tabular}

Figure S11.GPC data and distribution plot of cis-PB degraded PB (Table 1. Entry 10)

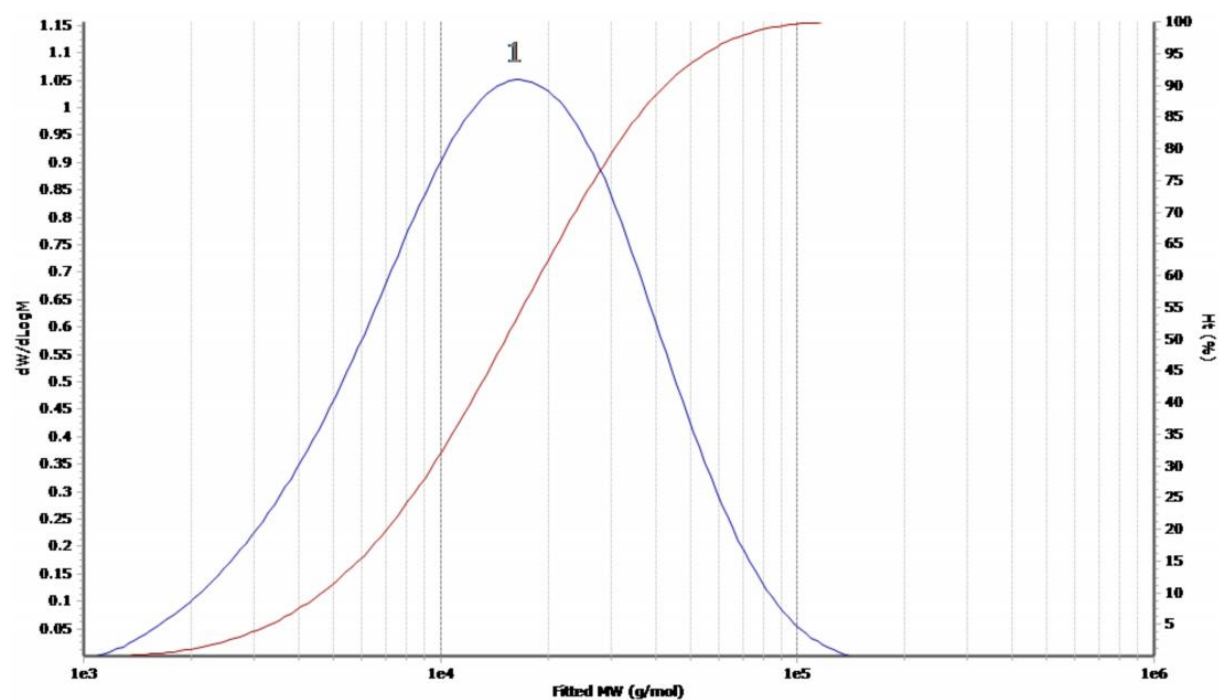

Molecular Weight Averages

\begin{tabular}{|c|c|r|r|r|r|r|r|}
\hline Peak & Mp & Mn & Mw & Mz & \multicolumn{1}{|c|}{ Mz+1 } & Mv & PD \\
\hline Peak 1 & 16429 & 10099 & 20309 & 34816 & 50574 & 32602 & 2.011 \\
\hline
\end{tabular}

Figure S12.GPC data and distribution plot of cis-PB degraded PB (Table 1. Entry 11) 


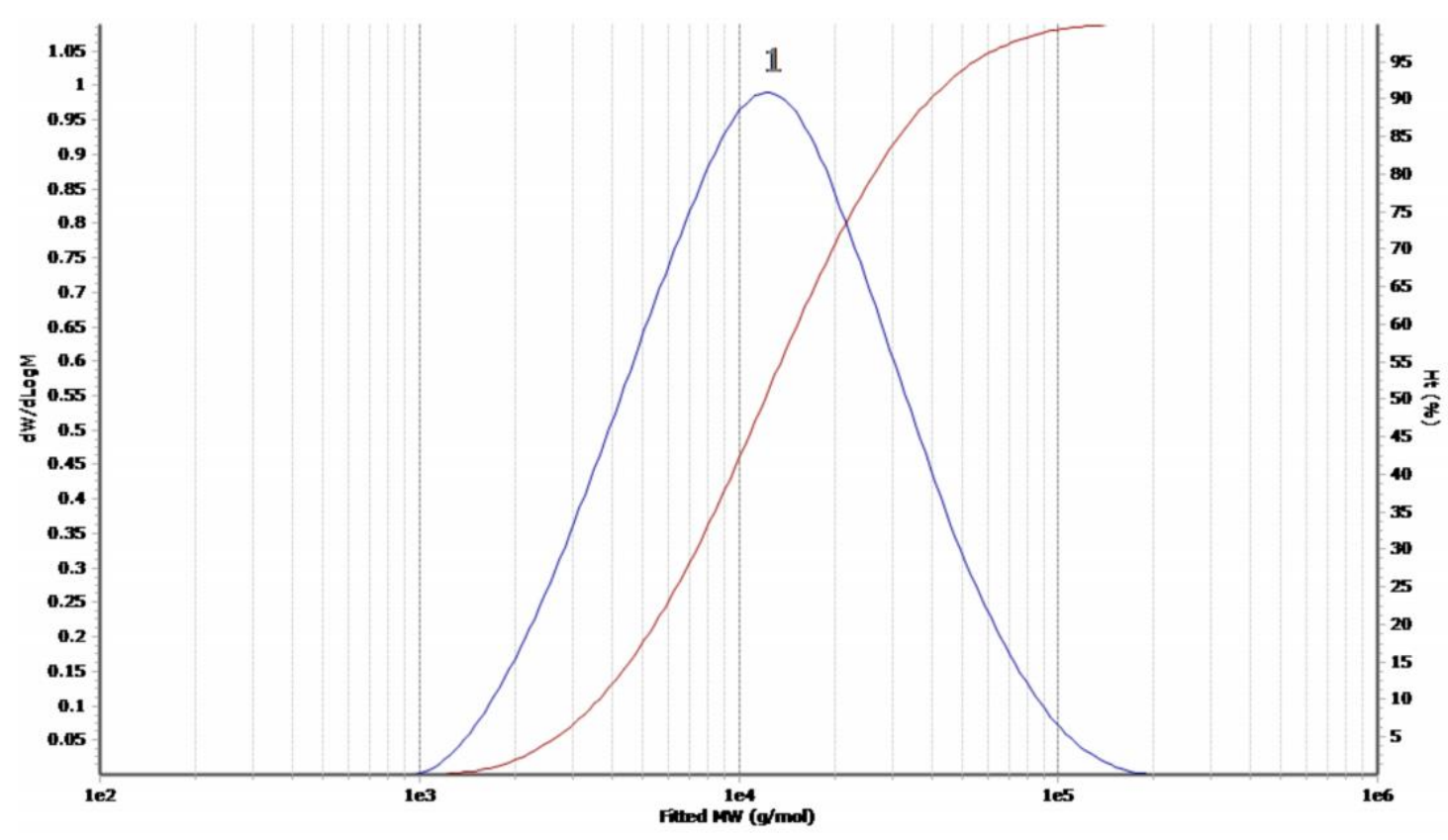

Molecular Weight Averages

\begin{tabular}{|c|c|c|c|c|c|c|c|}
\hline \begin{tabular}{|l} 
Peak \\
\end{tabular} & Mp & Mn & \begin{tabular}{|l|}
$\mathbf{M w}$ \\
\end{tabular} & \begin{tabular}{|l|l}
$\mathbf{M z}$ \\
\end{tabular} & $\mathrm{Mz+1}$ & \begin{tabular}{|l|l} 
Mv \\
\end{tabular} & PD \\
\hline Peak 1 & 12305 & 8128 & 18028 & 36740 & 60768 & 33582 & 2.218 \\
\hline
\end{tabular}

Figure S13.GPC data and distribution plot of cis-PB degraded PB (Table 1. Entry 12)

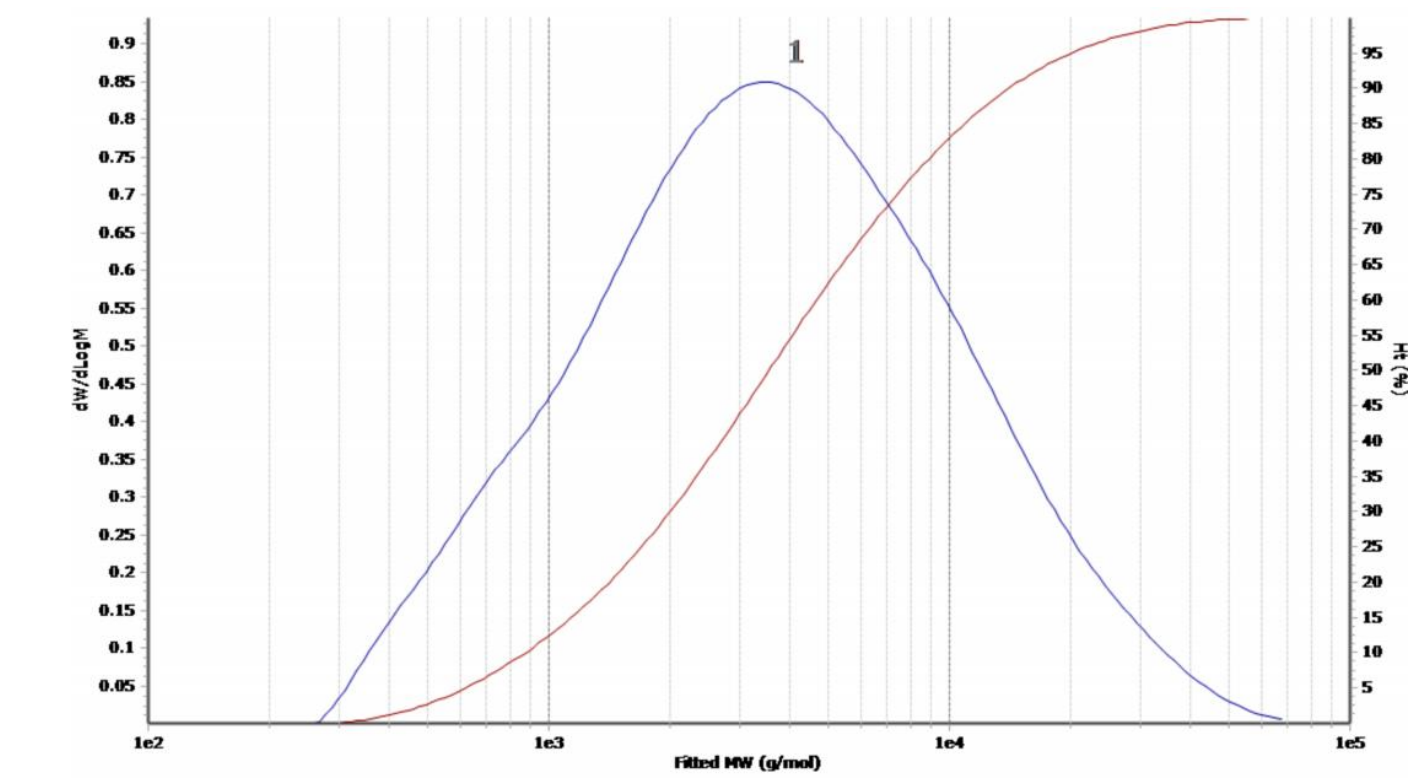

Molecular Weight Averages

\begin{tabular}{|c|r|r|r|r|r|r|r|}
\hline Peak & Mp & Mn & Mw & Mz & \multicolumn{1}{|c|}{ Mz+1 } & \multicolumn{1}{c|}{ Mv } & PD \\
\hline Peak 1 & 3475 & 2118 & 6000 & 14382 & 24794 & 12987 & 2.833 \\
\hline
\end{tabular}

Figure S14.GPC data and distribution plot of cis-PB degraded PB (Table 1. Entry 13) 


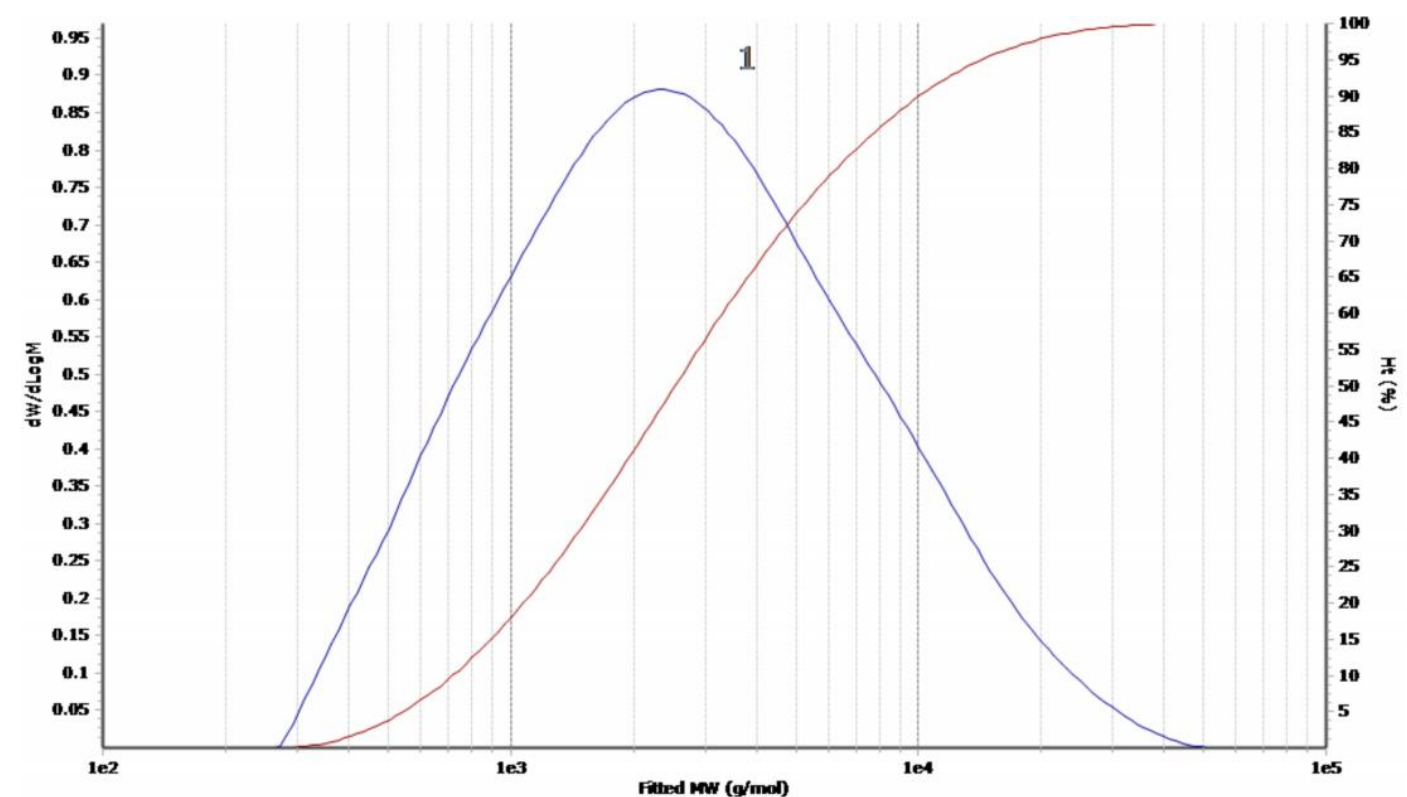

Molecular Weight Averages

\begin{tabular}{|c|c|r|r|r|r|r|r|}
\hline Peak & Mp & Mn & Mw & Mz & Mz+1 & Mv & PD \\
\hline Peak 1 & 2335 & 1686 & 4291 & 10081 & 17307 & 9108 & 2.545 \\
\hline
\end{tabular}

Figure S15.GPC data and distribution plot of cis-PB degraded PB (Table 1. Entry 14)

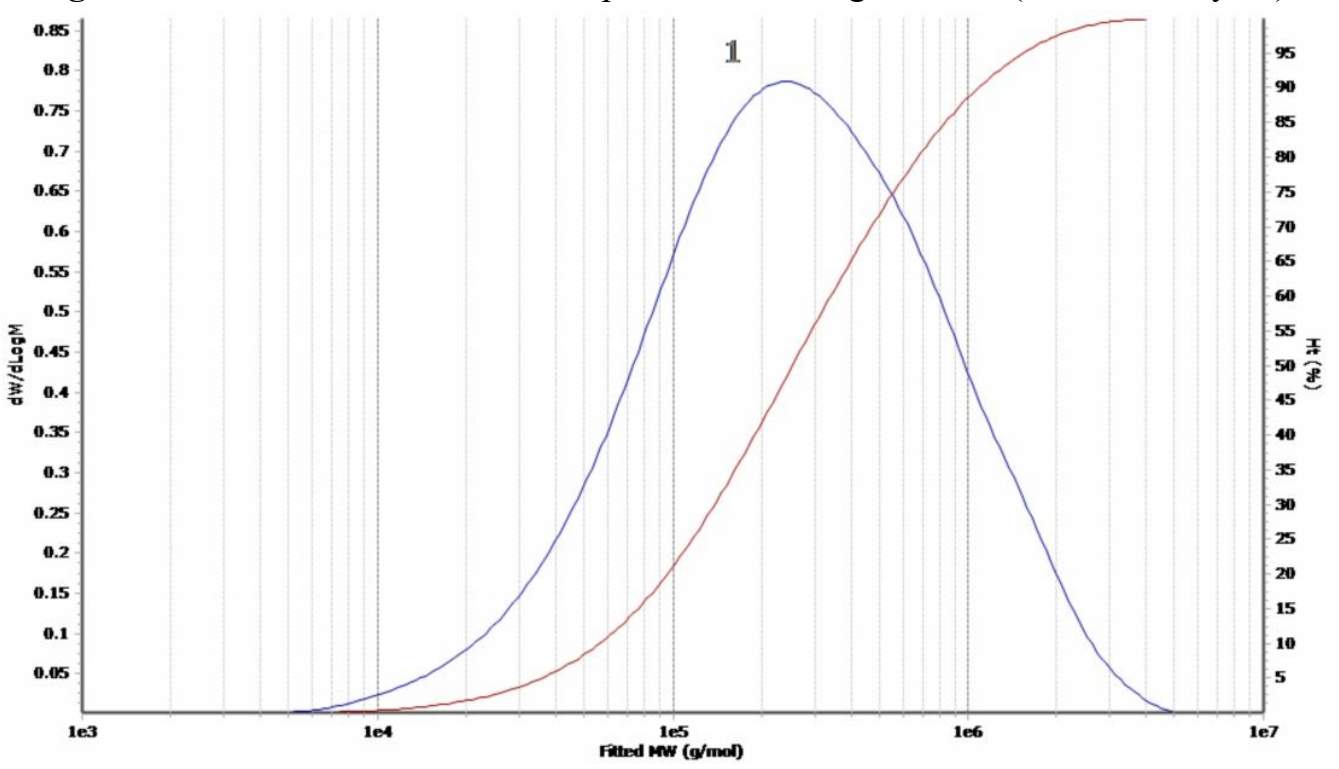

Molecular Weight Averages

\begin{tabular}{|c|c|c|c|c|r|r|r|}
\hline Peak & Mp & Mn & Mw & Mz & \multicolumn{1}{|c|}{ Mz+1 } & \multicolumn{1}{|c|}{ Mv } & PD \\
\hline Peak 1 & 238033 & 126893 & 444979 & 1076772 & 1795997 & 977469 & 3.507 \\
\hline
\end{tabular}

Figure S16.GPC data and distribution plot of cis-PB degraded PB (Table 1. Entry 15) 


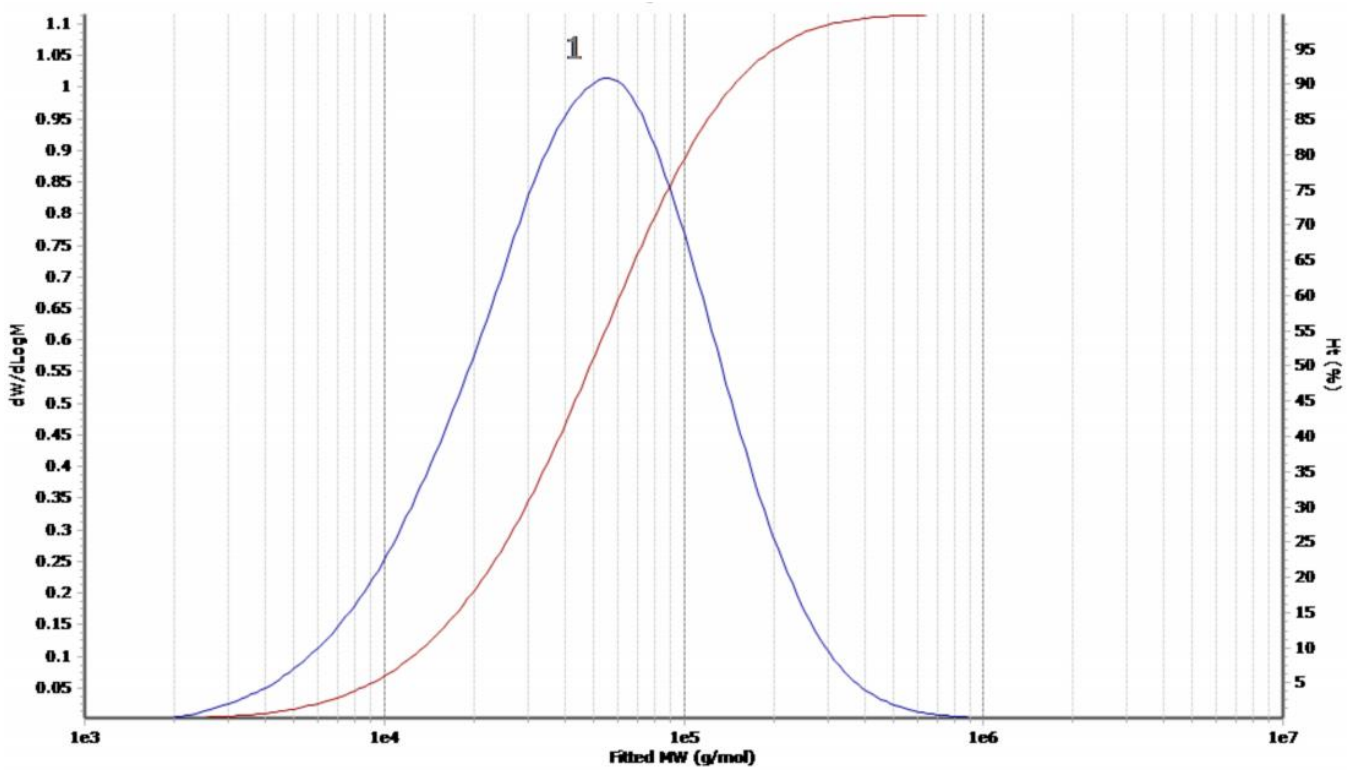

Molecular Weight Averages

\begin{tabular}{|c|c|c|c|c|c|c|r|}
\hline Peak & Mp & Mn & Mw & Mz & Mz+1 & Mv & PD \\
\hline Peak 1 & 56111 & 29293 & 69415 & 141549 & 258380 & 128429 & 2.37 \\
\hline
\end{tabular}

Figure S17.GPC data and distribution plot of cis-PB degraded PB (Table 1. Entry 16)

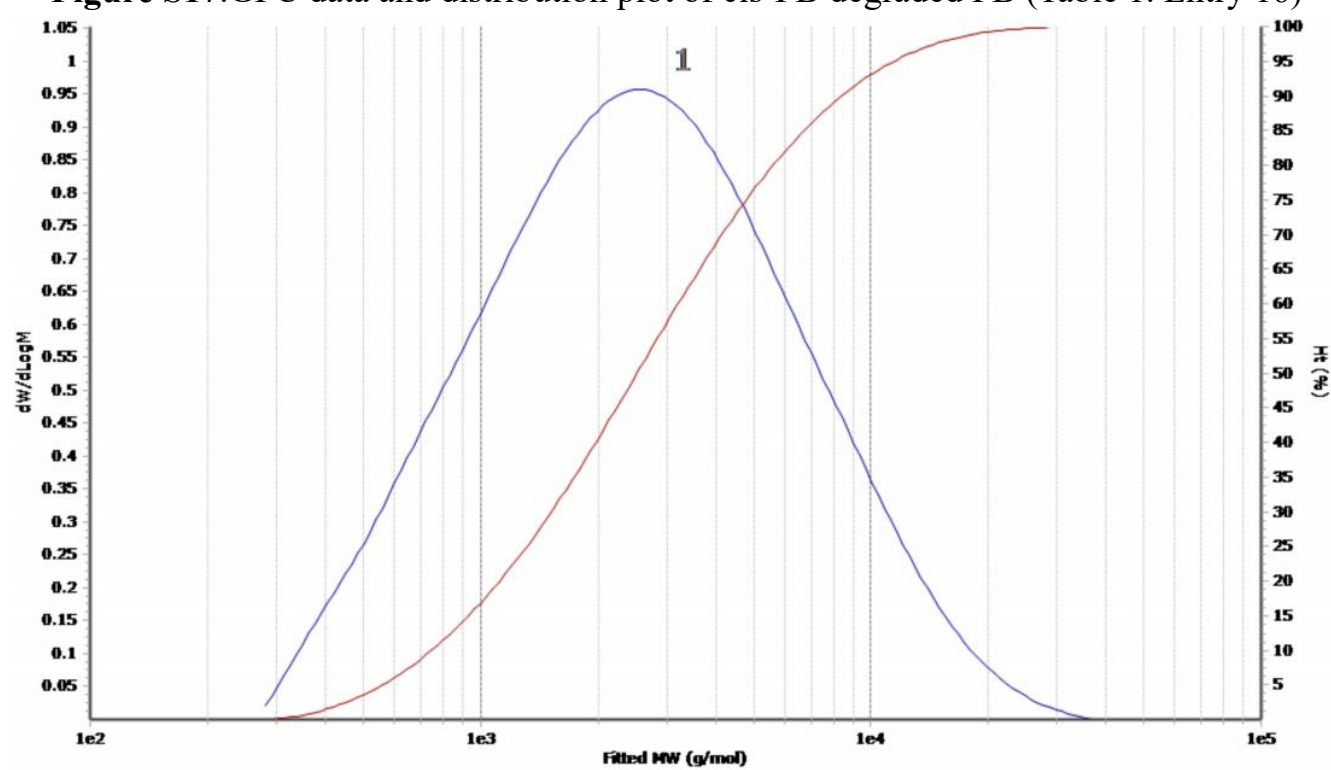

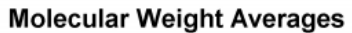

Molecular Weight Averages
\begin{tabular}{|c|c|r|r|r|r|r|r|}
\hline Peak & Mp & Mn & Mw & Mz & \multicolumn{1}{|c|}{ Mz+1 } & Mv & PD \\
\hline Peak 1 & 2556 & 1701 & 3776 & 7610 & 12326 & 6979 & 2.22 \\
\hline
\end{tabular}

Figure S18.GPC data and distribution plot of cis-PB degraded PB (Table 1. Entry 17) 


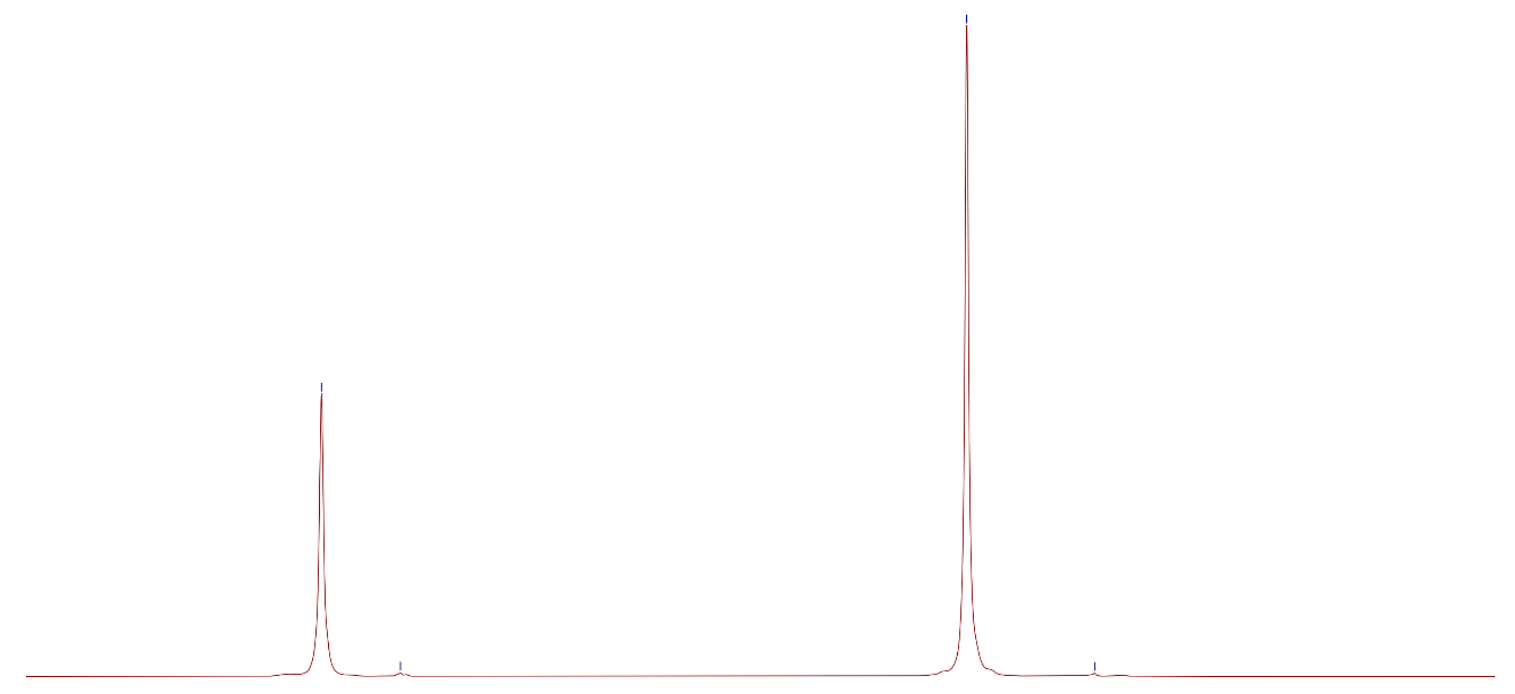

$\begin{array}{lllllllllllllll}6.5 & 6.0 & 5.5 & 5.0 & 4.5 & 4.0 & 3.5 & 3.0 & 2.5 & 2.0 & 1.5 & 1.0 & 0.5 & 0.0 & -0.5\end{array}$ Chemical shift (ppm)

Figure S19. ${ }^{1} \mathrm{H}-\mathrm{NMR}$ spectrum of blank control for cis-PB (reaction condition: in n-hexane, $80^{\circ} \mathrm{C}, 240 \mathrm{~min}$, no alkyllithium/TMEDA added)

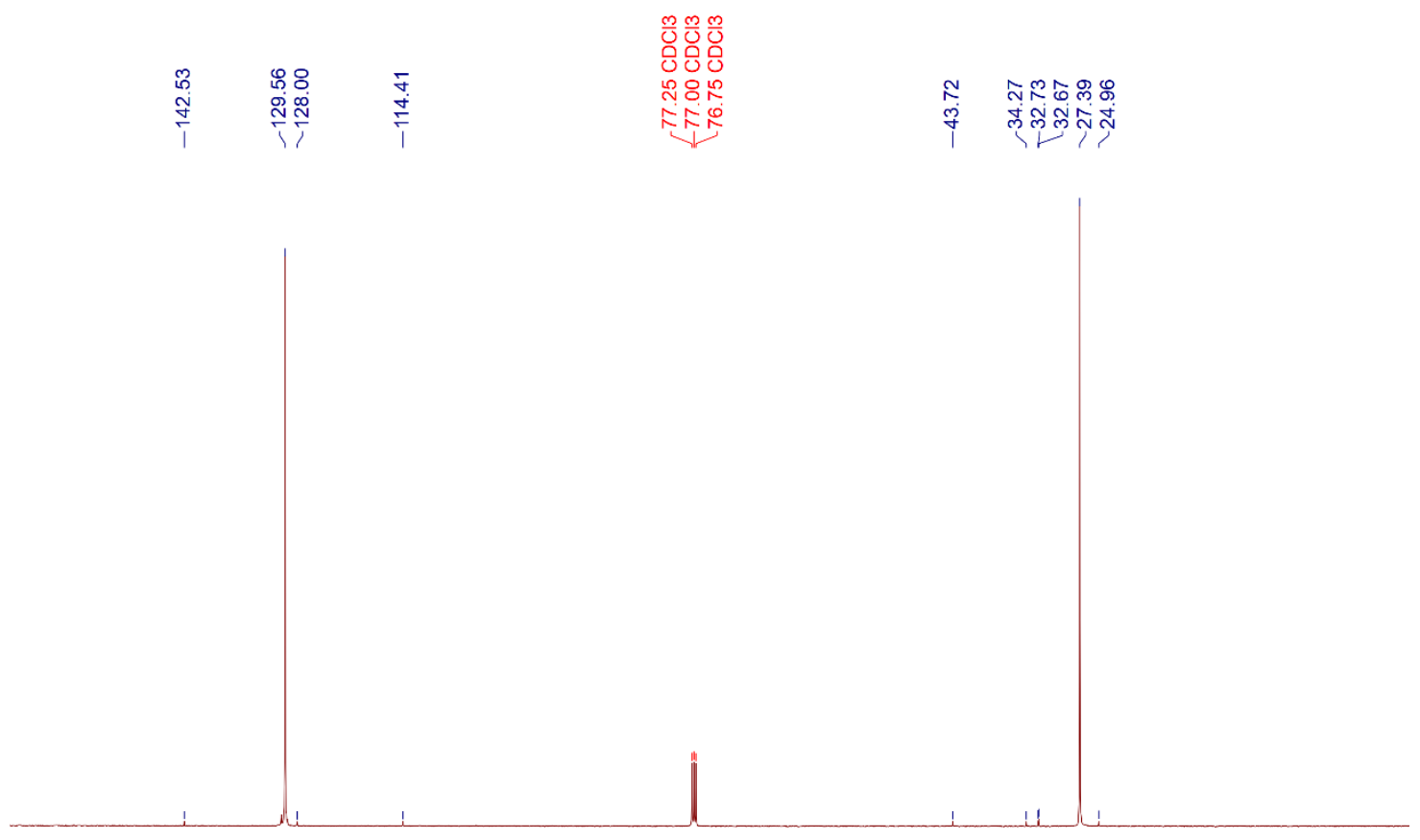

$\begin{array}{llllllllllllllllll}160 & 150 & 140 & 130 & 120 & 110 & 100 & 90 & 80 & 70 & 60 & 50 & 40 & 30 & 20 & 10 & 0 & -10\end{array}$ Chemical shift (ppm)

Figure S20. ${ }^{13} \mathrm{C}-\mathrm{NMR}$ spectrum of blank control for cis-PB 
(reaction condition: in n-hexane, $80^{\circ} \mathrm{C}, 240 \mathrm{~min}$, no alkyllithium/TMEDA added)

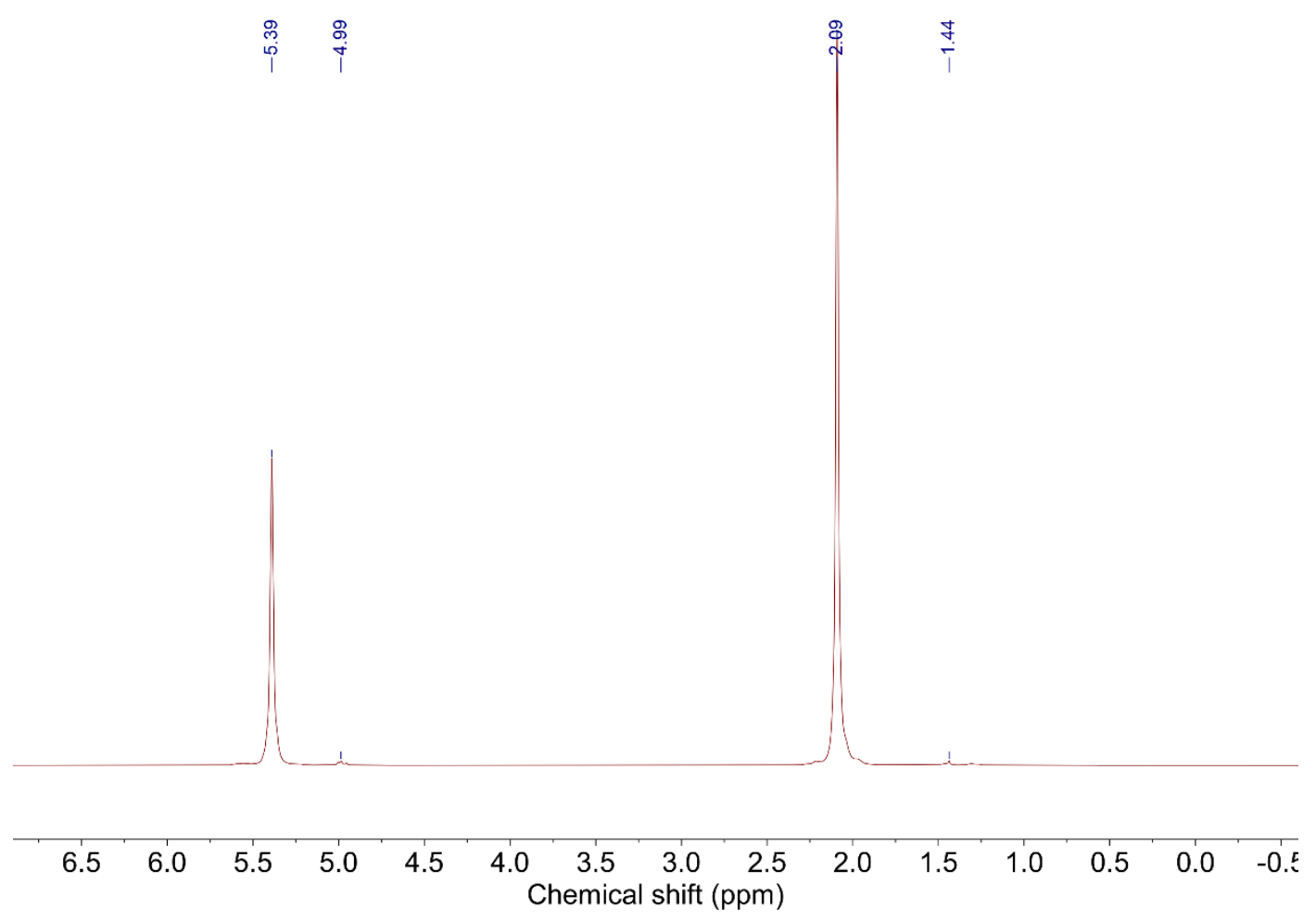

Figure S21. ${ }^{1} \mathrm{H}-\mathrm{NMR}$ spectrum for cis-PB (Table 1. Entry 1)

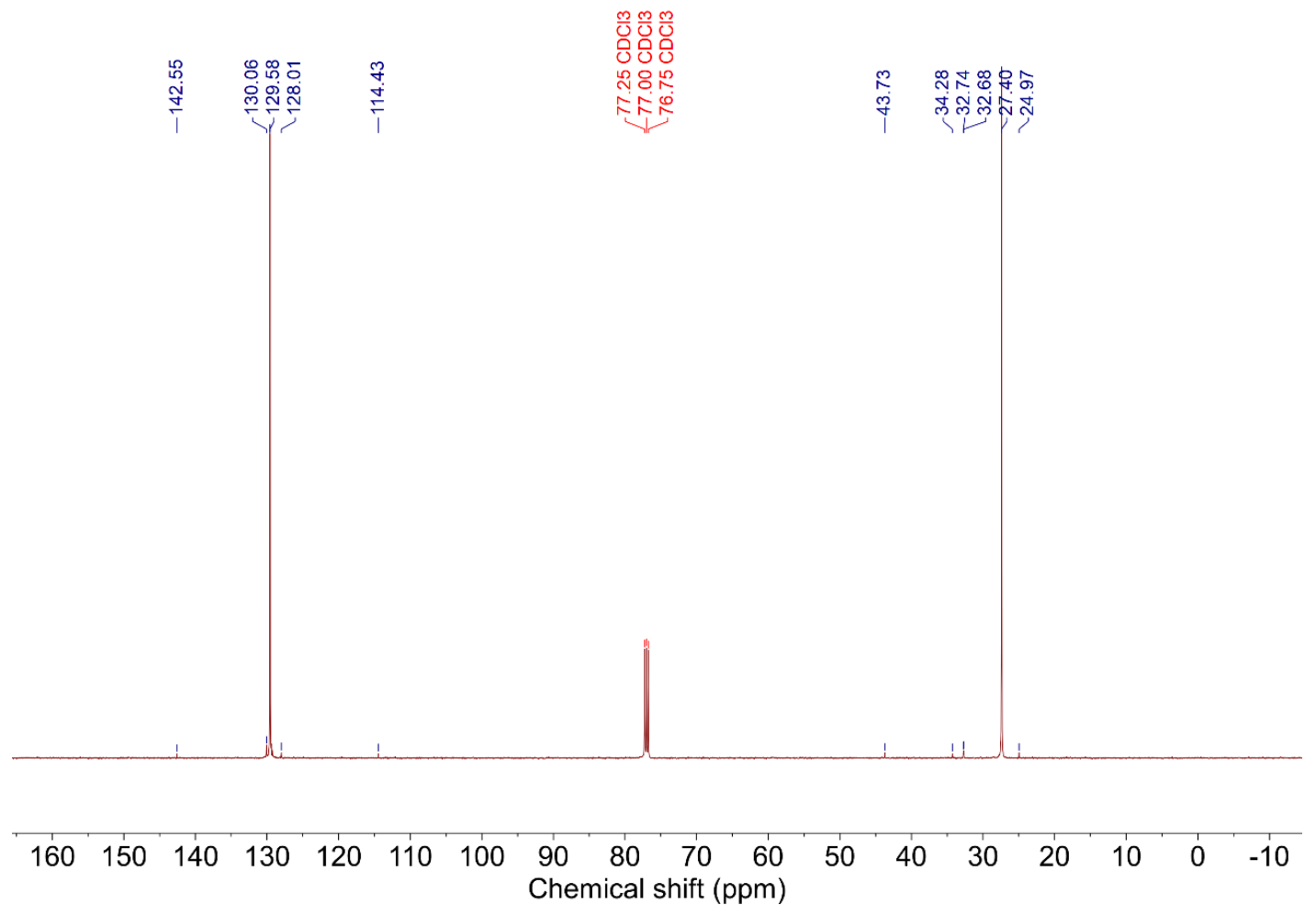

Figure S22. ${ }^{13} \mathrm{C}-\mathrm{NMR}$ spectrum for cis-PB (Table 1. Entry 1) 


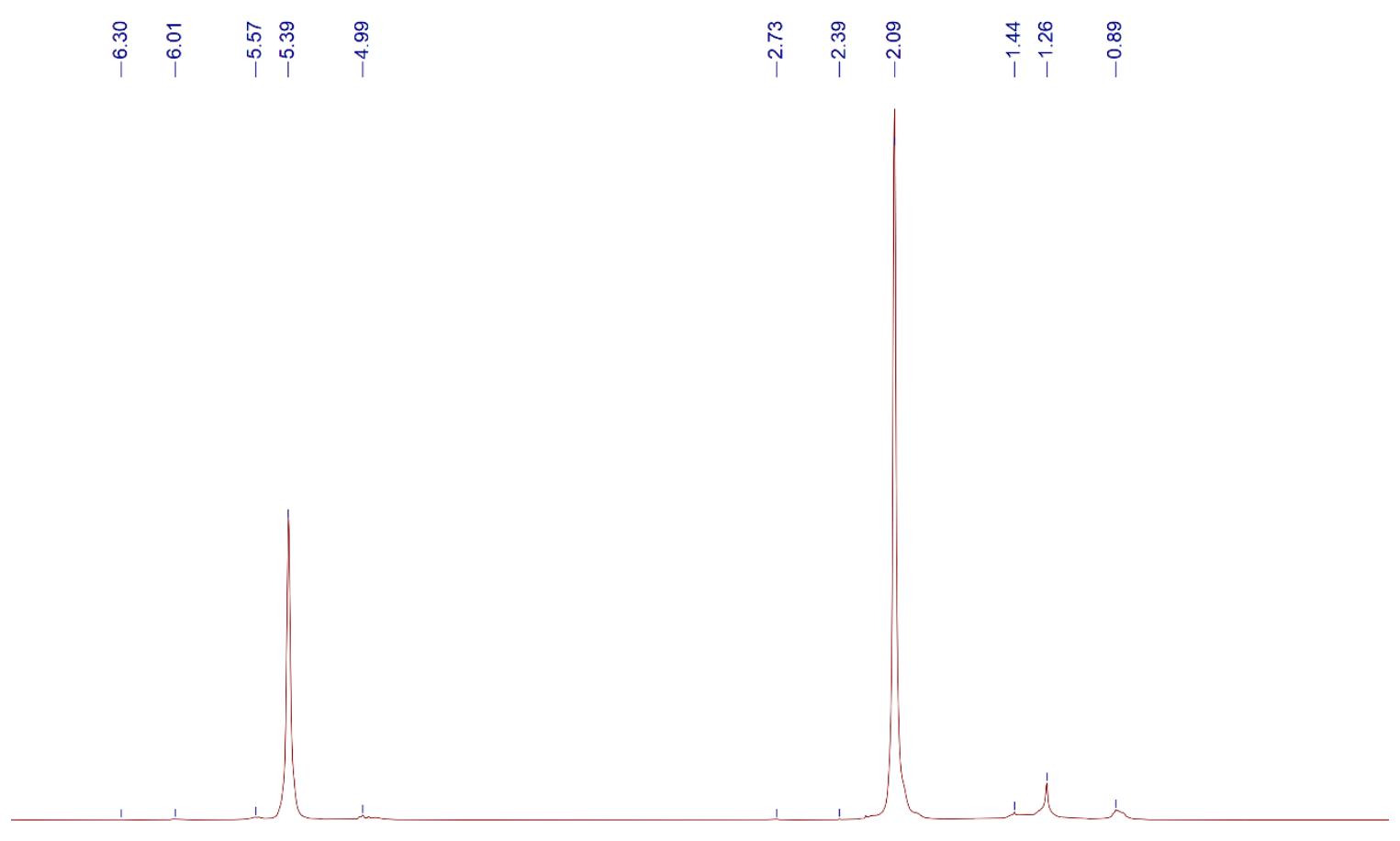

\begin{tabular}{|c|c|c|c|c|c|c|c|c|c|c|c|}
\hline 6.5 & 6.0 & 5.5 & 5.0 & 4.5 & 4.0 & $\begin{array}{c}3.5 \\
\text { iemica }\end{array}$ & $\begin{array}{l}3.0 \\
\text { shift }\end{array}$ & $\begin{array}{r}2.5 \\
\mathrm{ppm})\end{array}$ & 2.0 & 1.5 & 1. \\
\hline
\end{tabular}

Figure S23. ${ }^{1} \mathrm{H}-\mathrm{NMR}$ spectrum for degraded PB (Table 1. Entry 2)

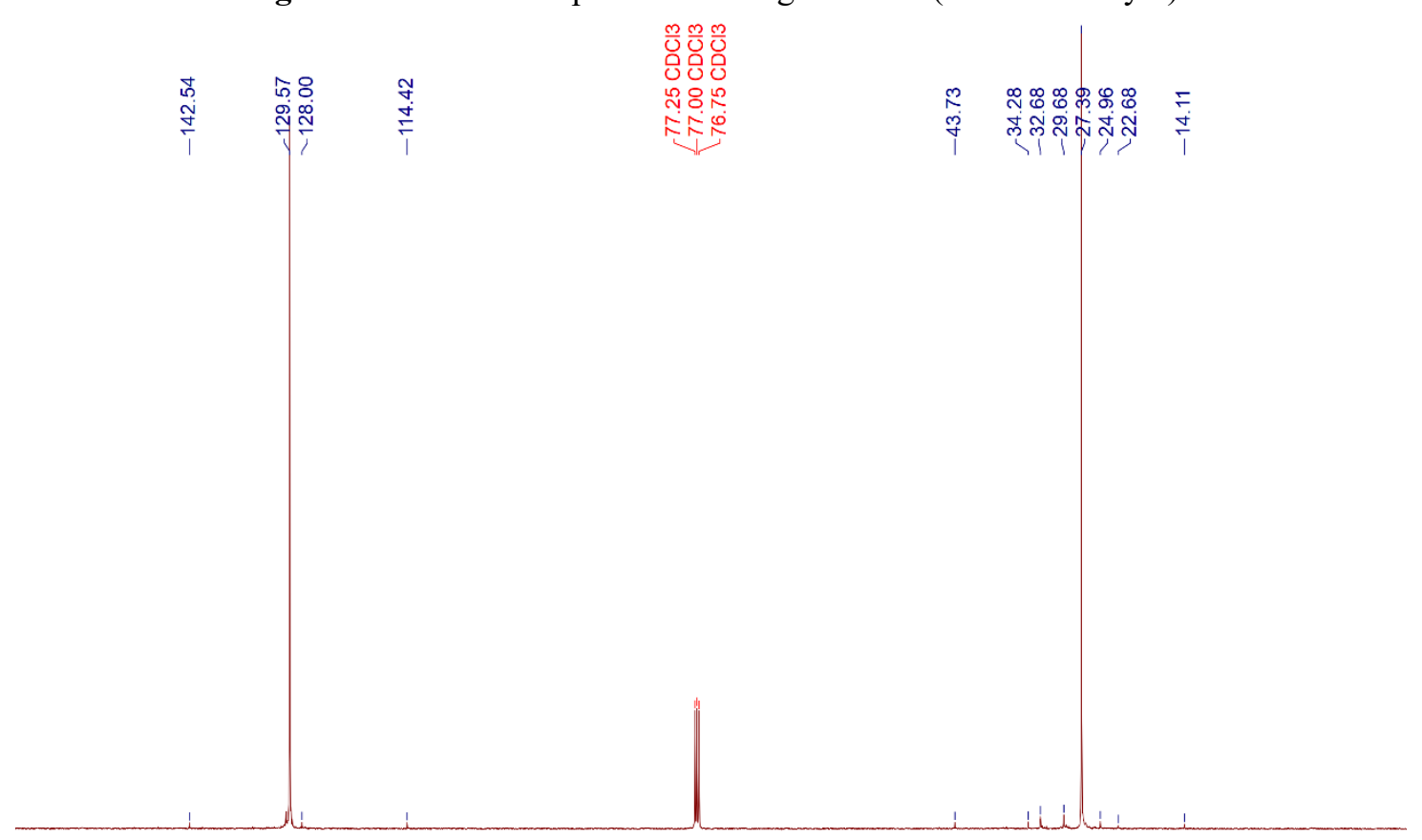

$\begin{array}{llllllllllllllllll}160 & 150 & 140 & 130 & 120 & 110 & 100 & \begin{array}{c}90 \\ \text { Chemical shift (ppm) }\end{array}\end{array}$

Figure S24. ${ }^{13}$ C-NMR spectrum for degraded PB (Table 1. Entry 2)

$15 / 65$ 


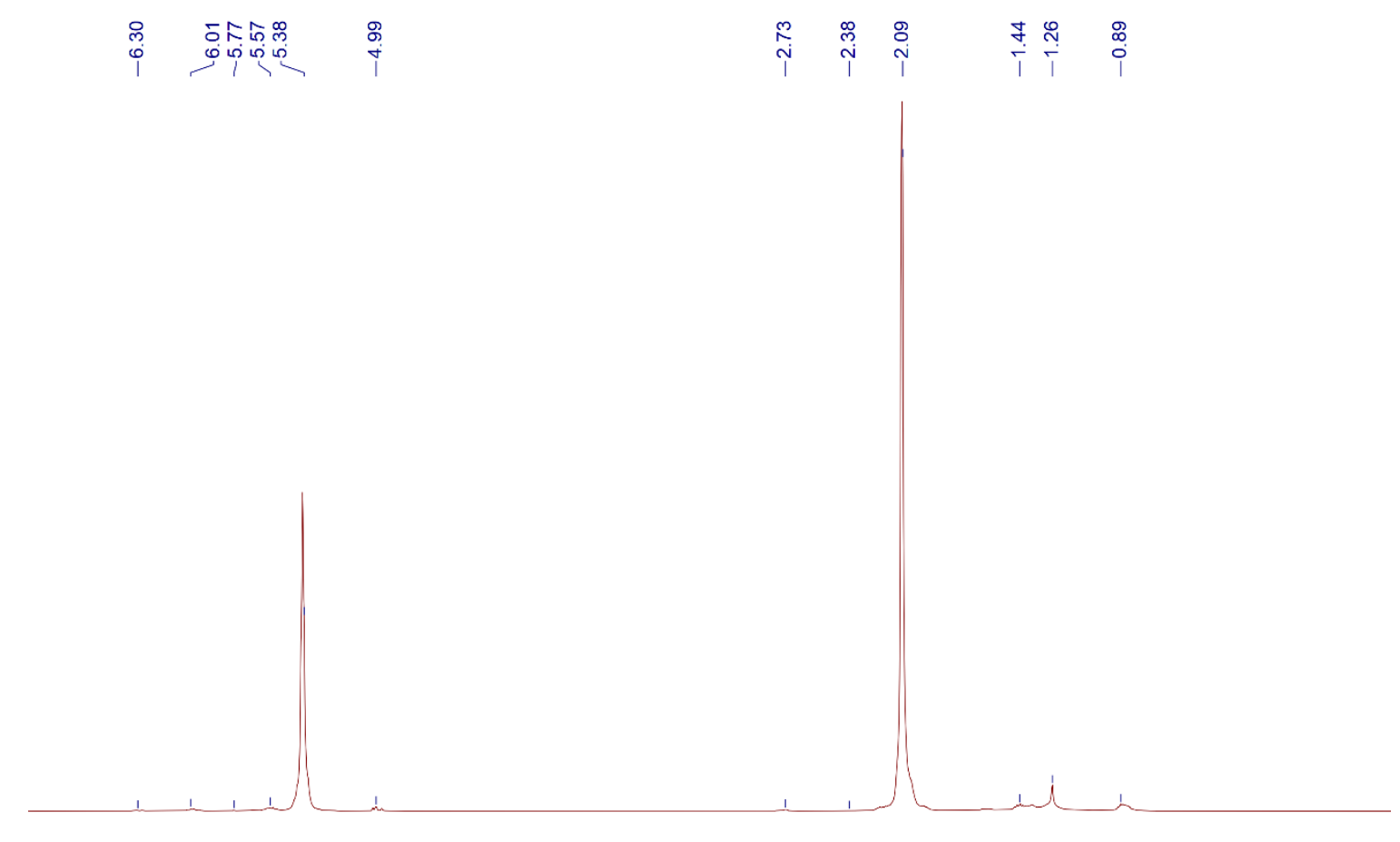

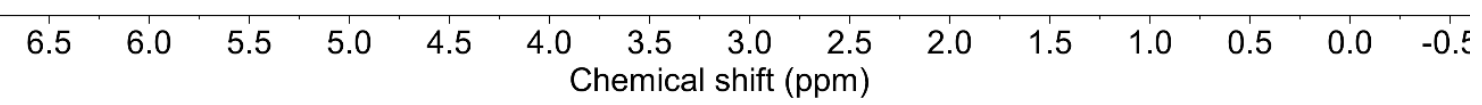

Figure S25. ${ }^{1} \mathrm{H}-\mathrm{NMR}$ spectrum for degraded PB (Table 1. Entry 3)
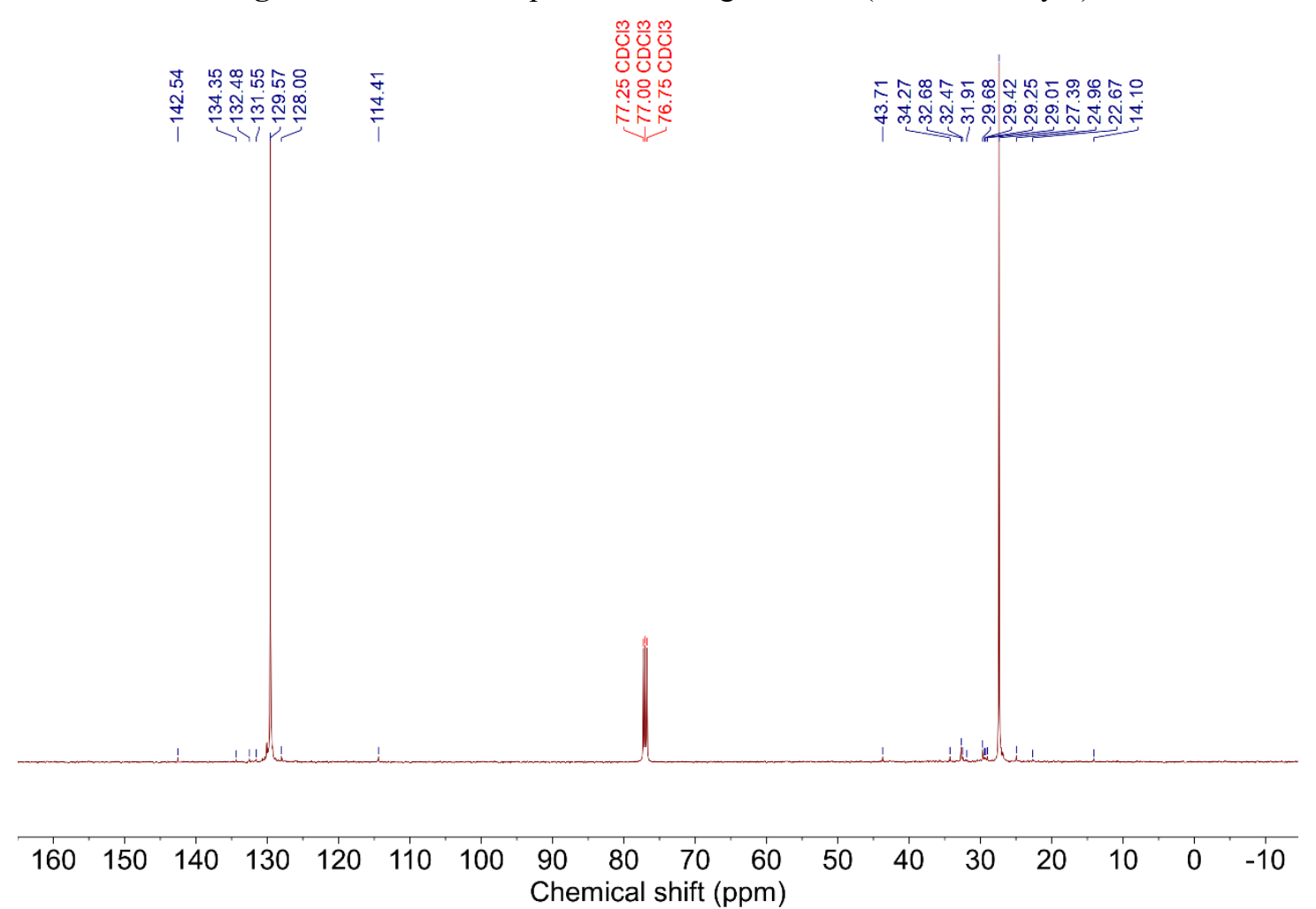

Figure S26. ${ }^{13} \mathrm{C}-\mathrm{NMR}$ spectrum for degraded PB (Table 1. Entry 3)

$16 / 65$ 


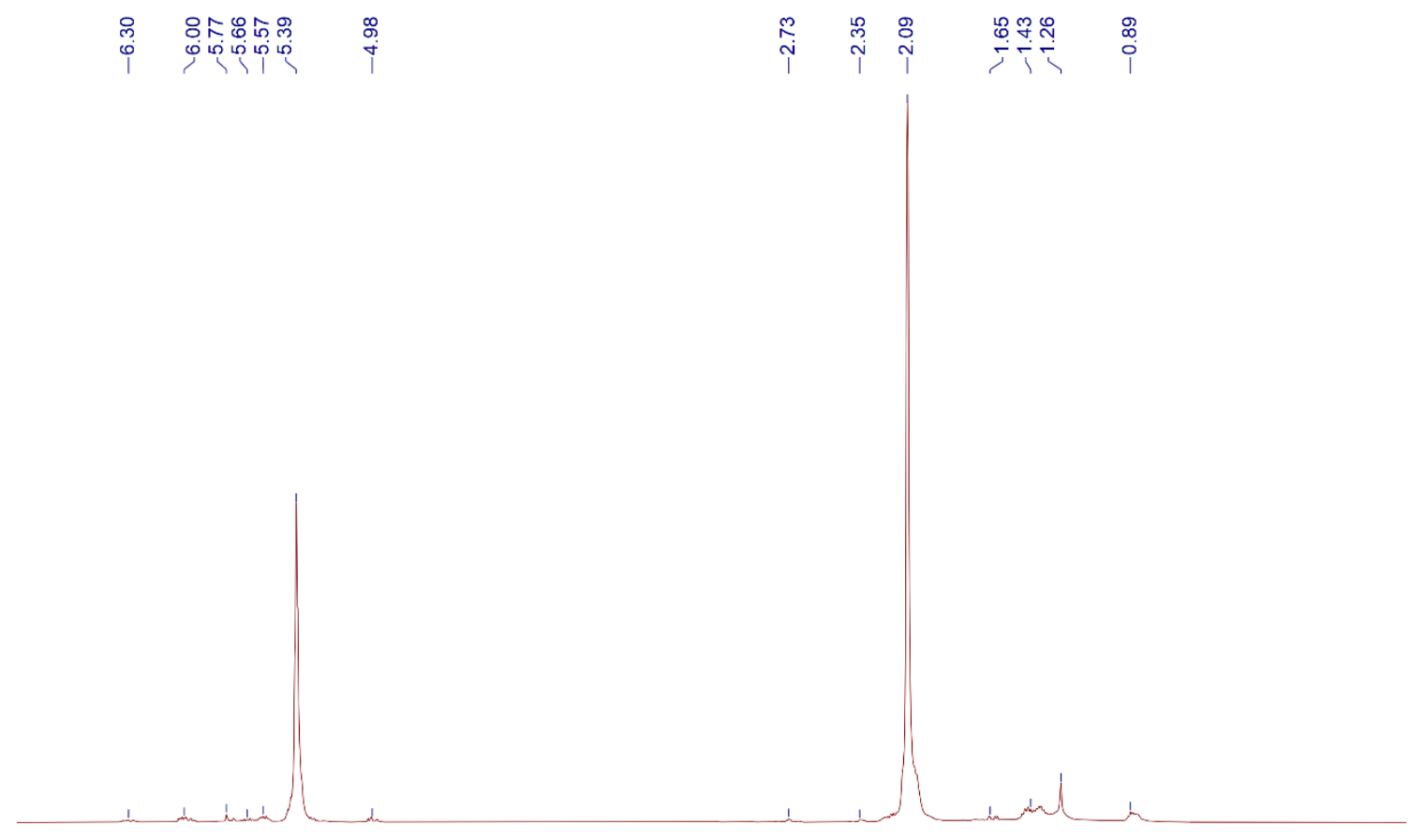

$\begin{array}{llllllllllllll}6.5 & 6.0 & 5.5 & 5.0 & 4.5 & 4.0 & \begin{array}{c}3.5 \\ \text { Chemical shift }\end{array} & \begin{array}{l}3.0 \\ (\mathrm{ppm})\end{array} & 2.0 & 1.5 & 1.0 & 0.5 & 0.0 & -0.5\end{array}$

Figure S27. ${ }^{1} \mathrm{H}-\mathrm{NMR}$ spectrum for degraded PB (Table 1. Entry 4)

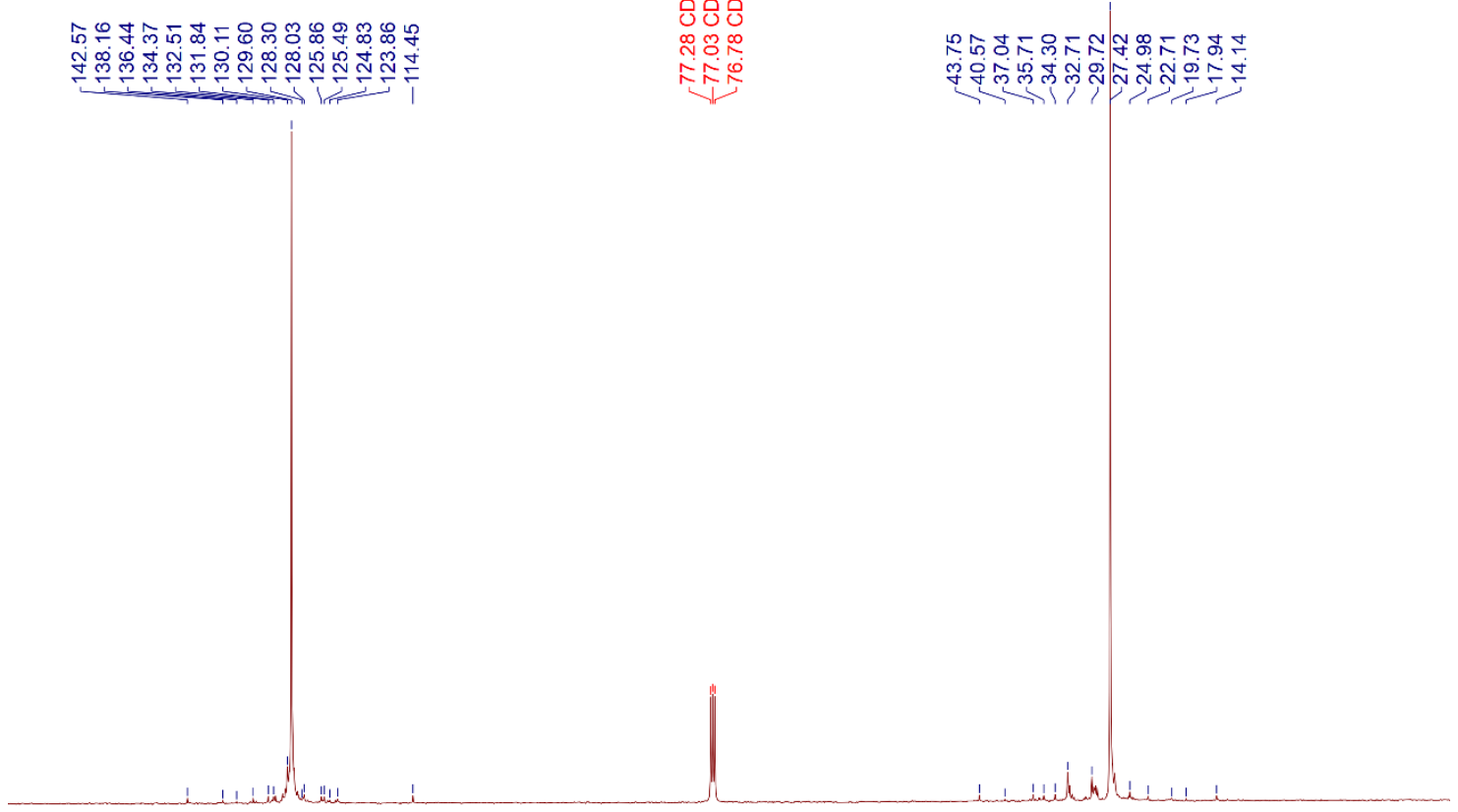

$\begin{array}{llllllllllllllllll}160 & 150 & 140 & 130 & 120 & 110 & 100 & \begin{array}{c}90 \\ \text { Chemical shift (ppm) }\end{array} & & 80 & 70 & 60 & 50 & 40 & 20 & 10 & 0 & -10\end{array}$

Figure S28. ${ }^{13} \mathrm{C}-\mathrm{NMR}$ spectrum for degraded PB (Table 1. Entry 4)

$17 / 65$ 


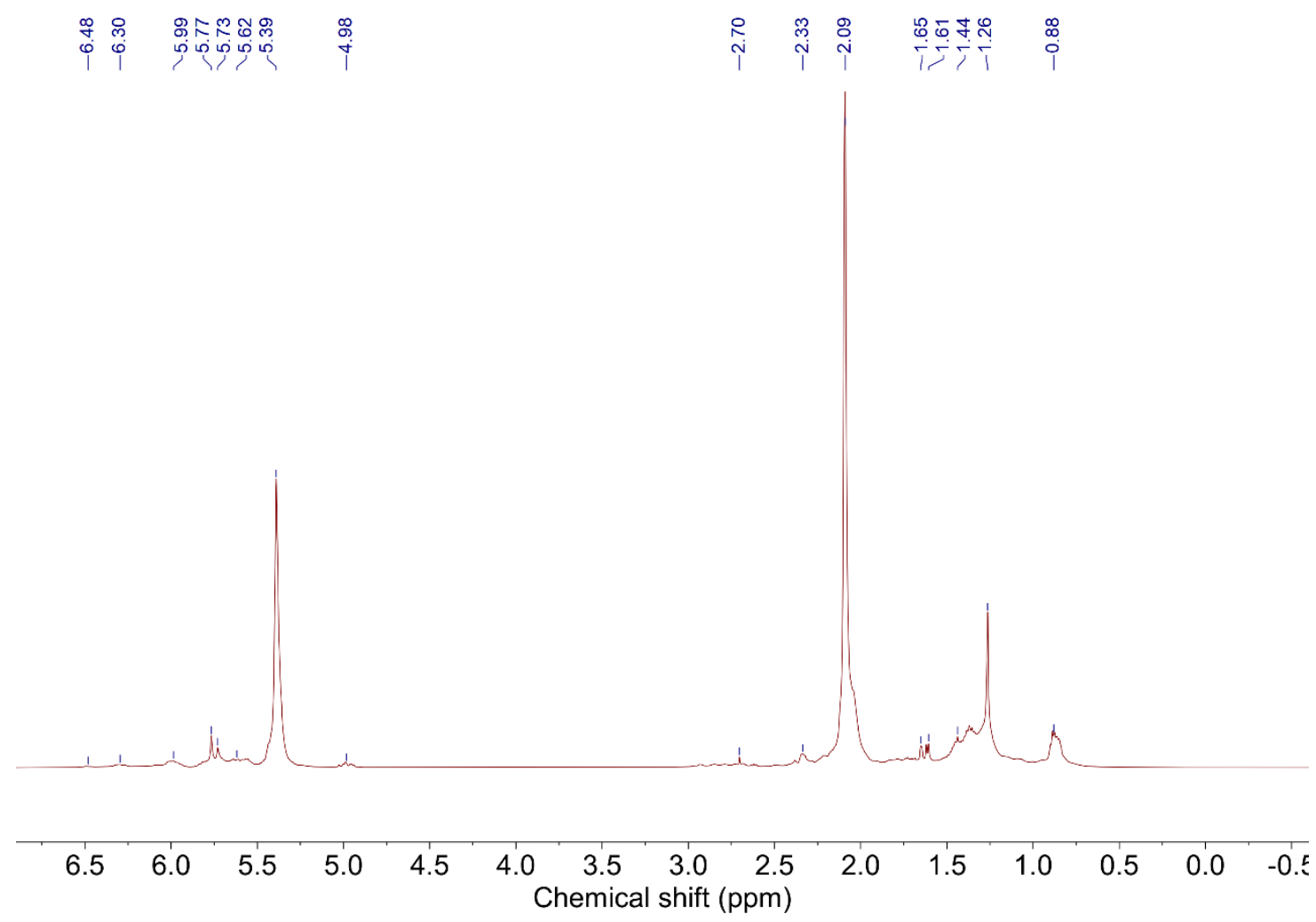

Figure S29. ${ }^{1} \mathrm{H}-\mathrm{NMR}$ spectrum for degraded PB (Table 1. Entry 5)
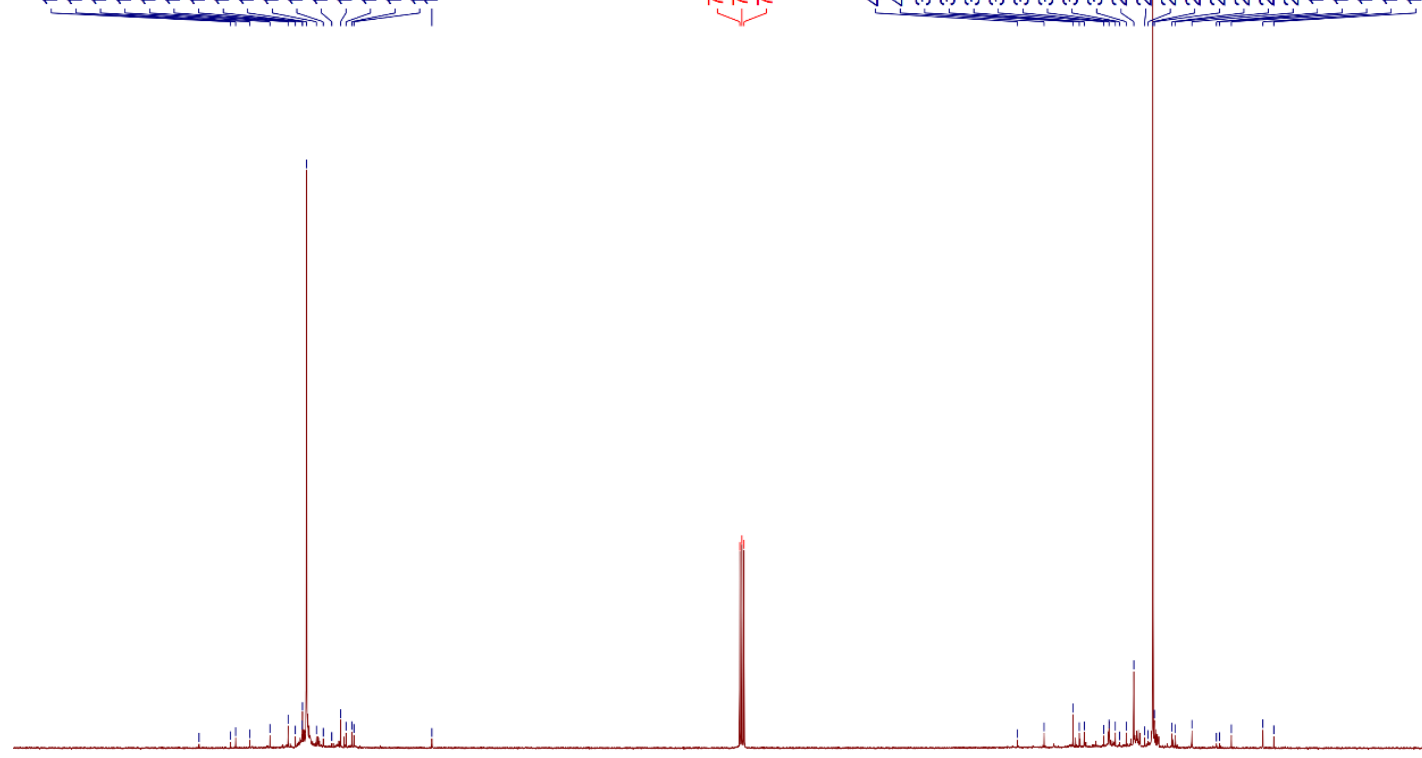

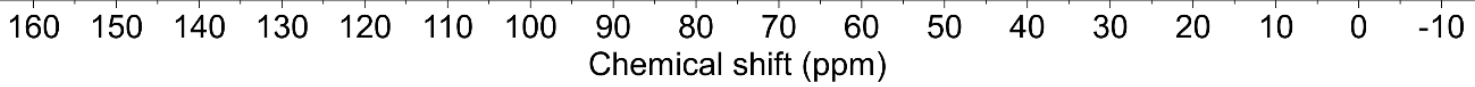

Figure S30. ${ }^{13} \mathrm{C}-\mathrm{NMR}$ spectrum for degraded PB (Table 1. Entry 5) 


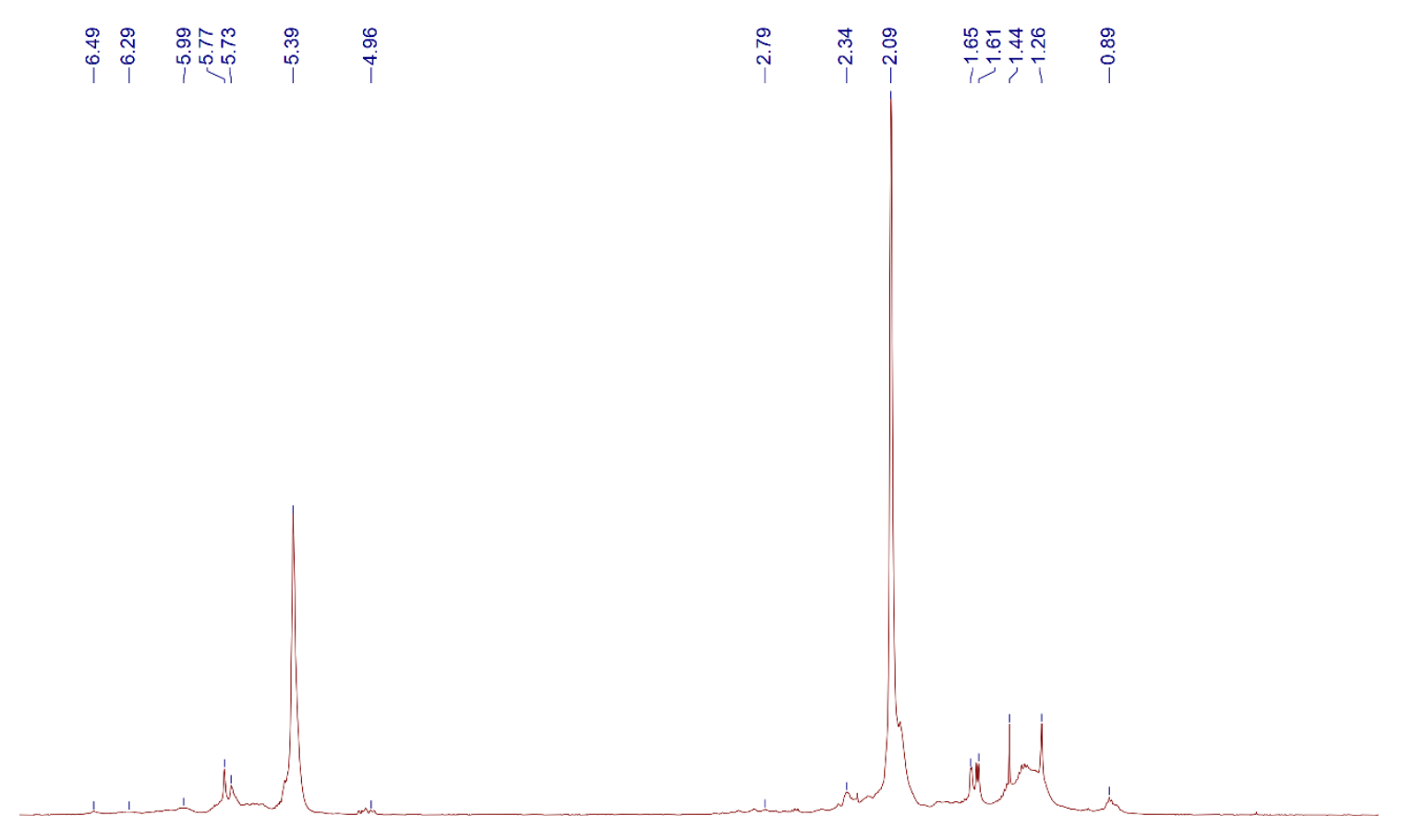

\section{$\begin{array}{lllllllllllllll}6.5 & 6.0 & 5.5 & 5.0 & 4.5 & 4.0 & 3.5 & 3.0 & 2.5 & 2.0 & 1.5 & 1.0 & 0.5 & 0.0 & -0.5\end{array}$ Chemical shift (ppm)}

Figure S31. ${ }^{1} \mathrm{H}-\mathrm{NMR}$ spectrum for degraded PB (Table 1. Entry 6)
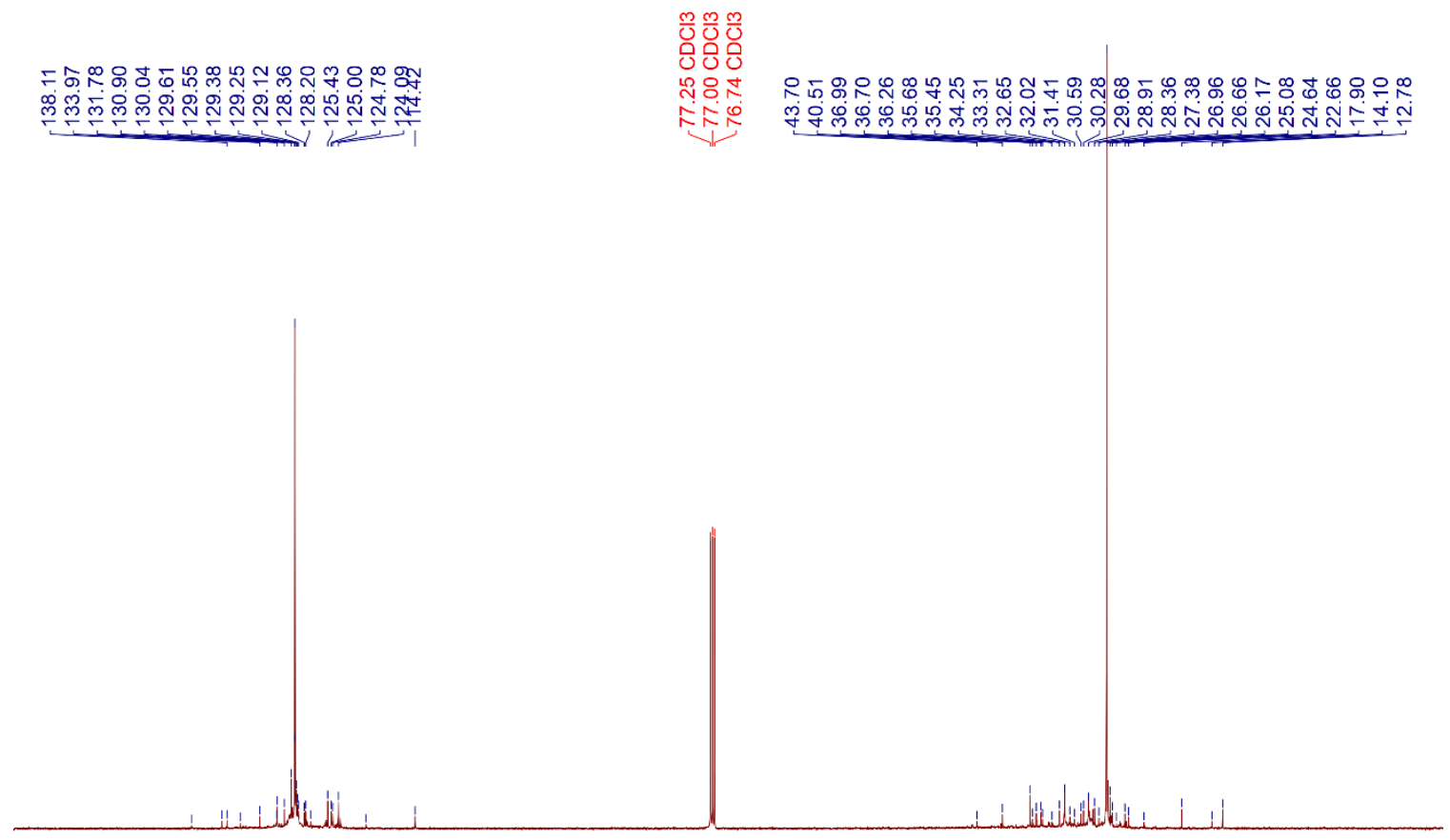

$\begin{array}{llllllllllllllllll}160 & 150 & 140 & 130 & 120 & 110 & 100 & \begin{array}{c}90 \\ \text { Chemical shift (ppm) }\end{array}\end{array}$

Figure S32. ${ }^{13} \mathrm{C}-\mathrm{NMR}$ spectrum for degraded PB (Table 1. Entry 6) 


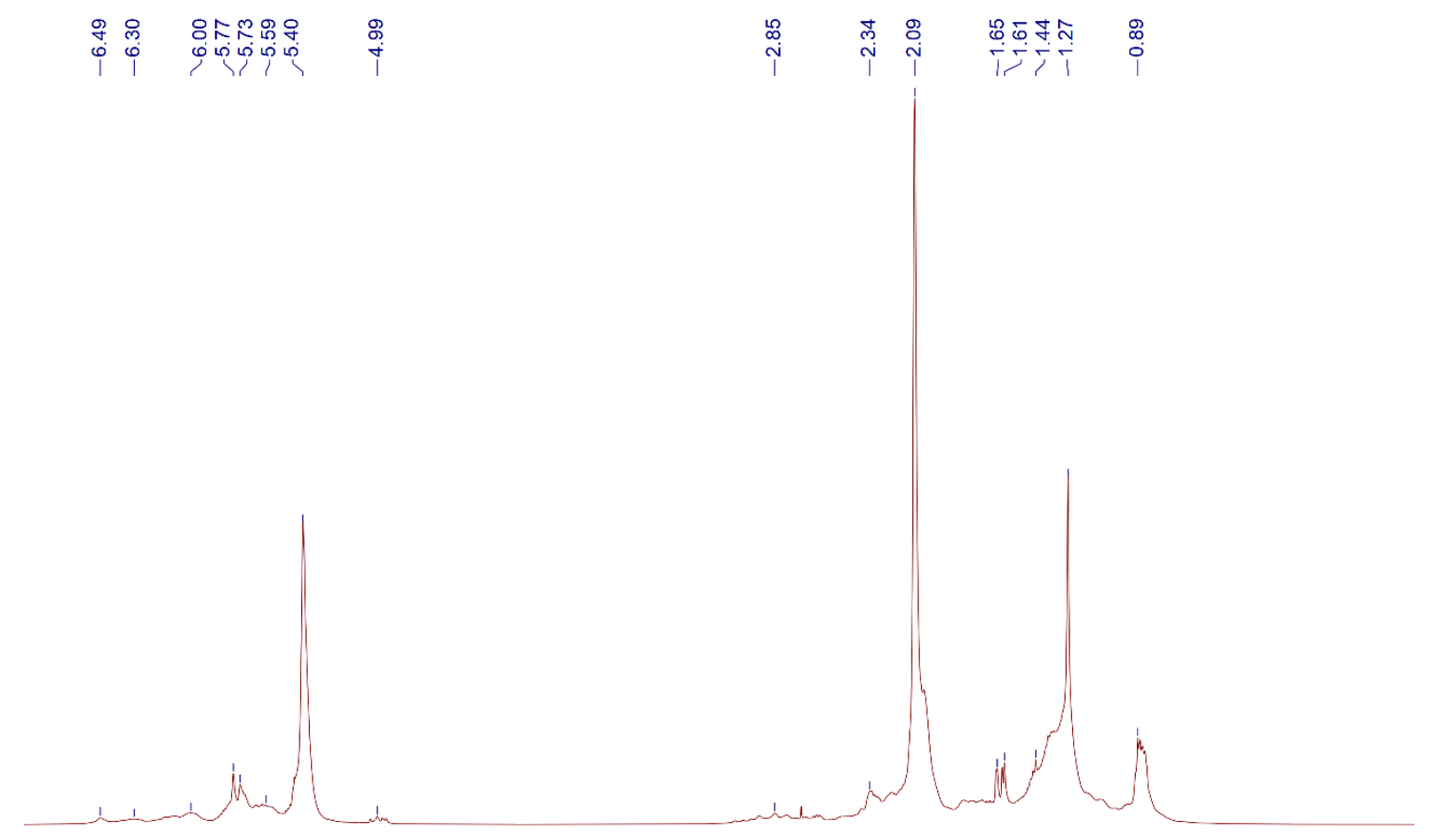

$\begin{array}{llllllllllllll}6.5 & 6.0 & 5.5 & 5.0 & 4.5 & 4.0 & \begin{array}{c}3.5 \\ \text { Chemical shift (ppm) }\end{array} & 2.0 & 2.5 & 1.5 & 1.0 & 0.5 & 0.0 & -0.5\end{array}$

Figure S33. ${ }^{1} \mathrm{H}-\mathrm{NMR}$ spectrum for degraded PB (Table 1. Entry 7)

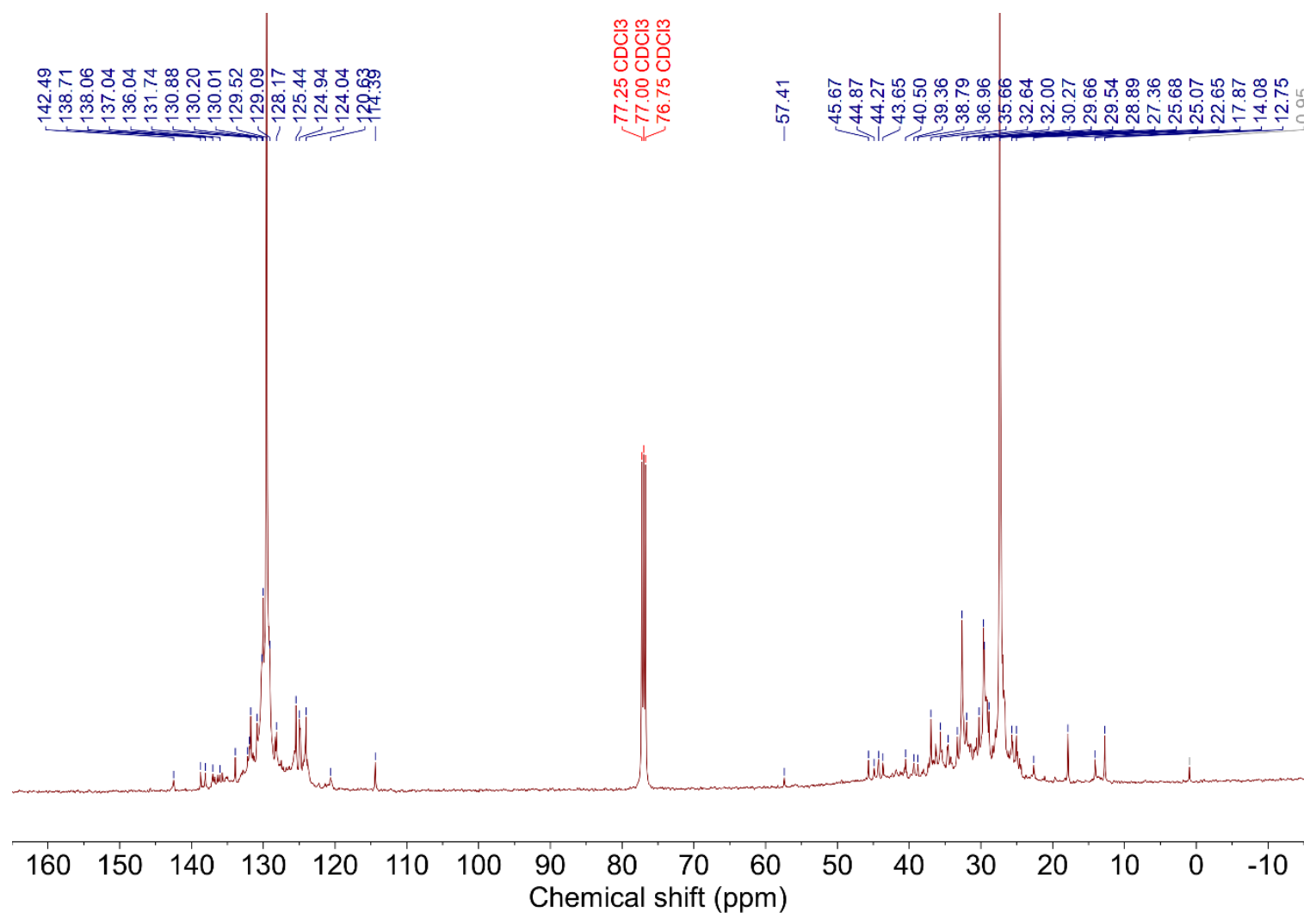

Figure S34. ${ }^{13}$ C-NMR spectrum for degraded PB (Table 1. Entry 7) 


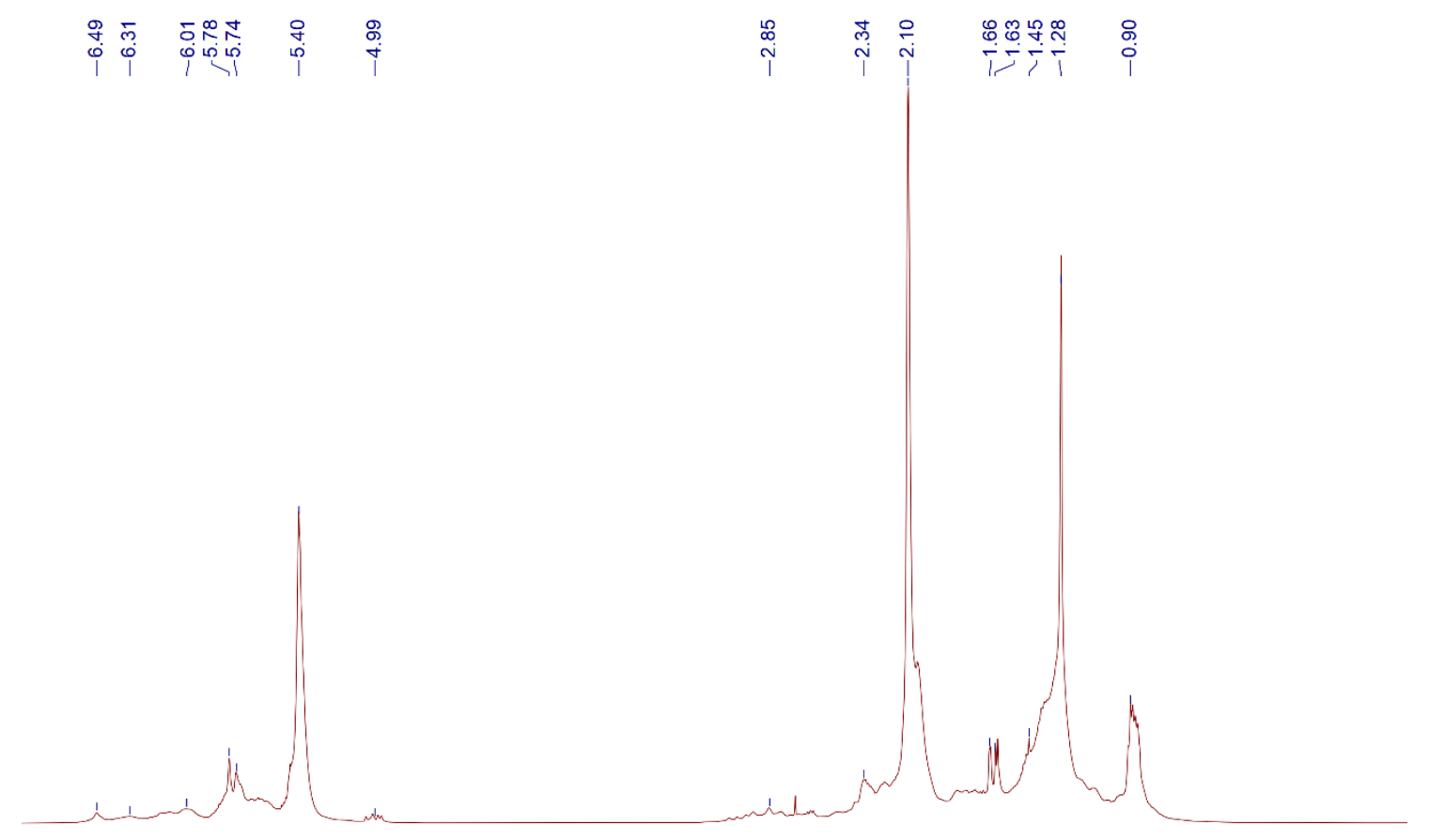

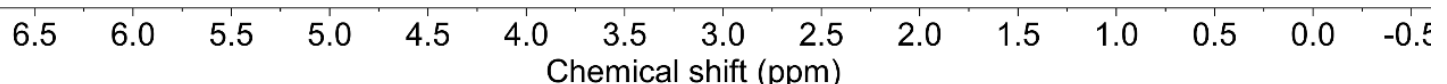

Figure S35. ${ }^{1} \mathrm{H}-\mathrm{NMR}$ spectrum for degraded PB (Table 1. Entry 8)
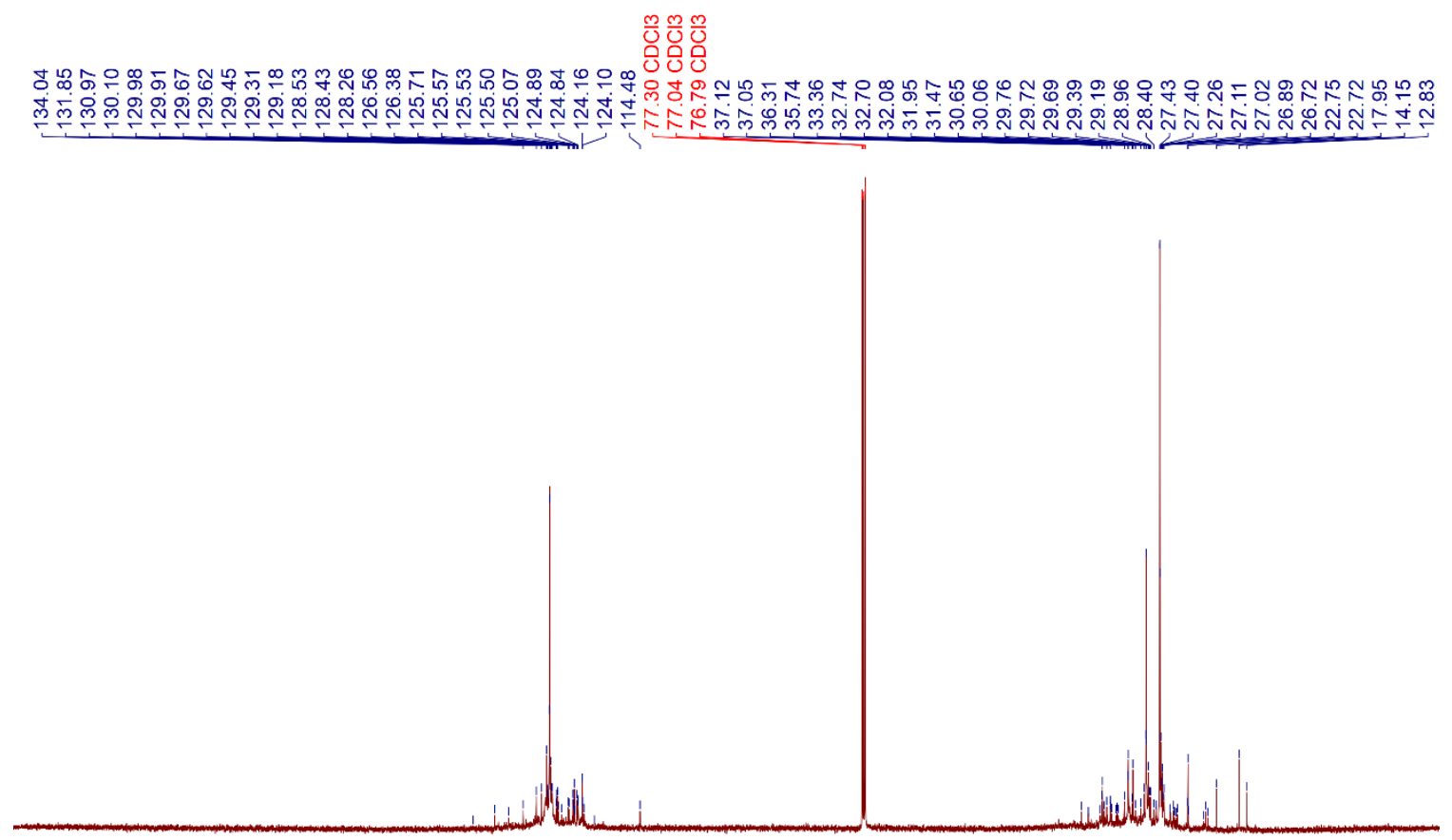

$21020019018017016015014013012011010090 \quad 8070 \quad 6050 \quad 40 \begin{array}{lllllll}1 & 20 & 10 & 0 & -10\end{array}$ Chemical shift (ppm)

Figure S36. ${ }^{13}$ C-NMR spectrum for degraded PB (Table 1. Entry 8) 


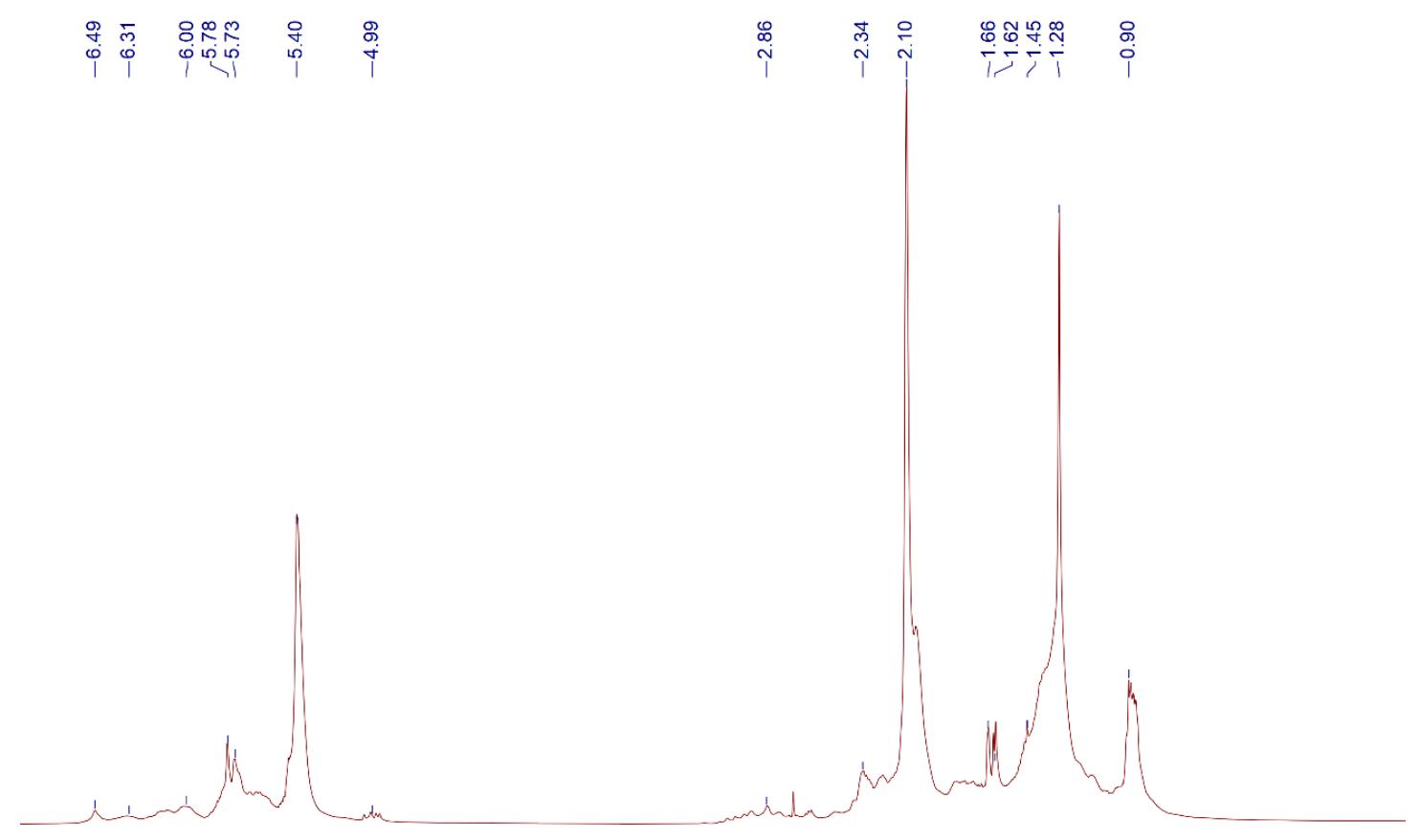

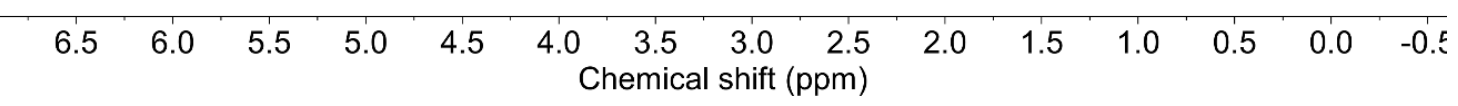

Figure S37. ${ }^{1} \mathrm{H}-\mathrm{NMR}$ spectrum for degraded PB (Table 1. Entry 9)
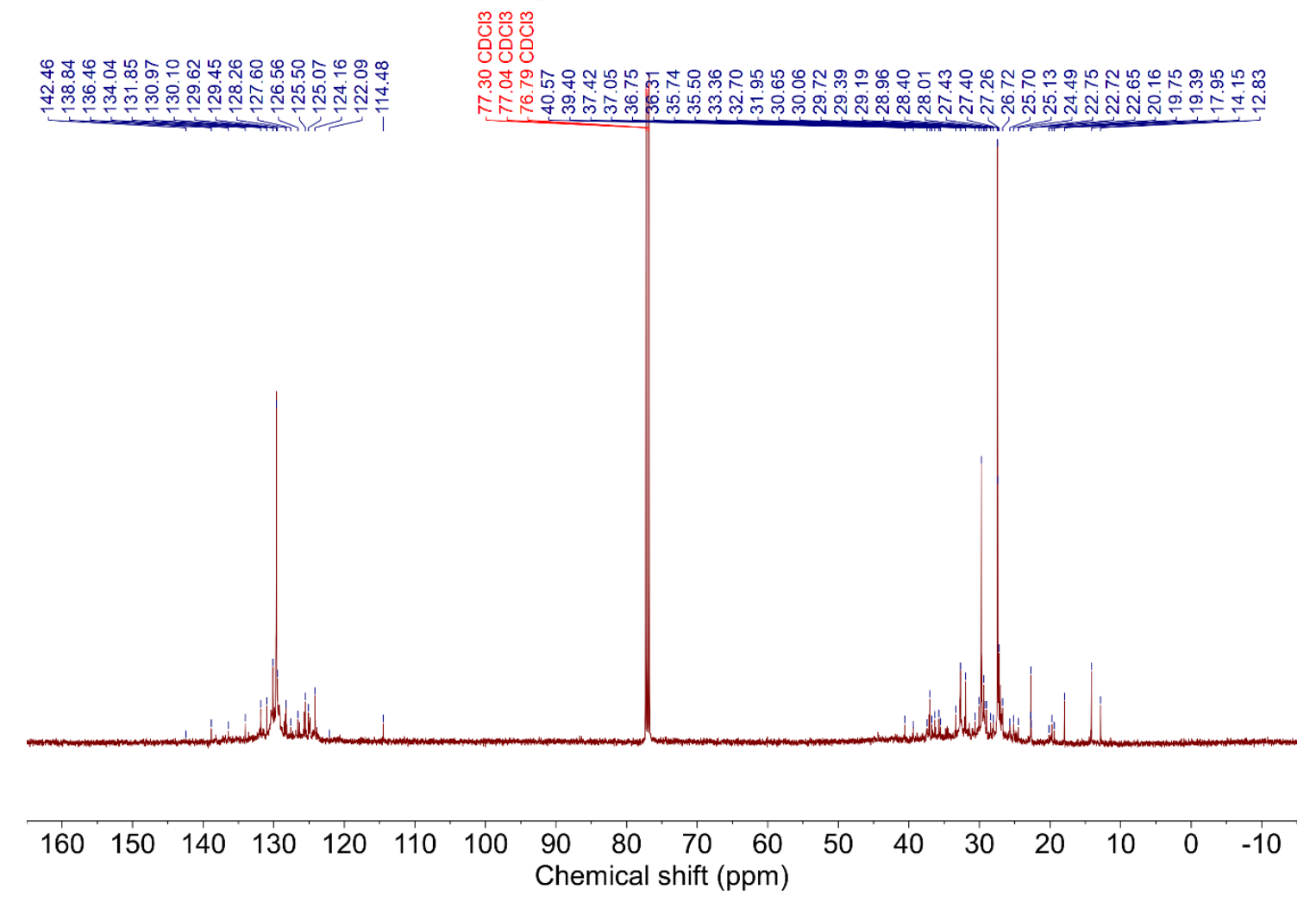

Figure S38. ${ }^{13}$ C-NMR spectrum for degraded PB (Table 1. Entry 9) 


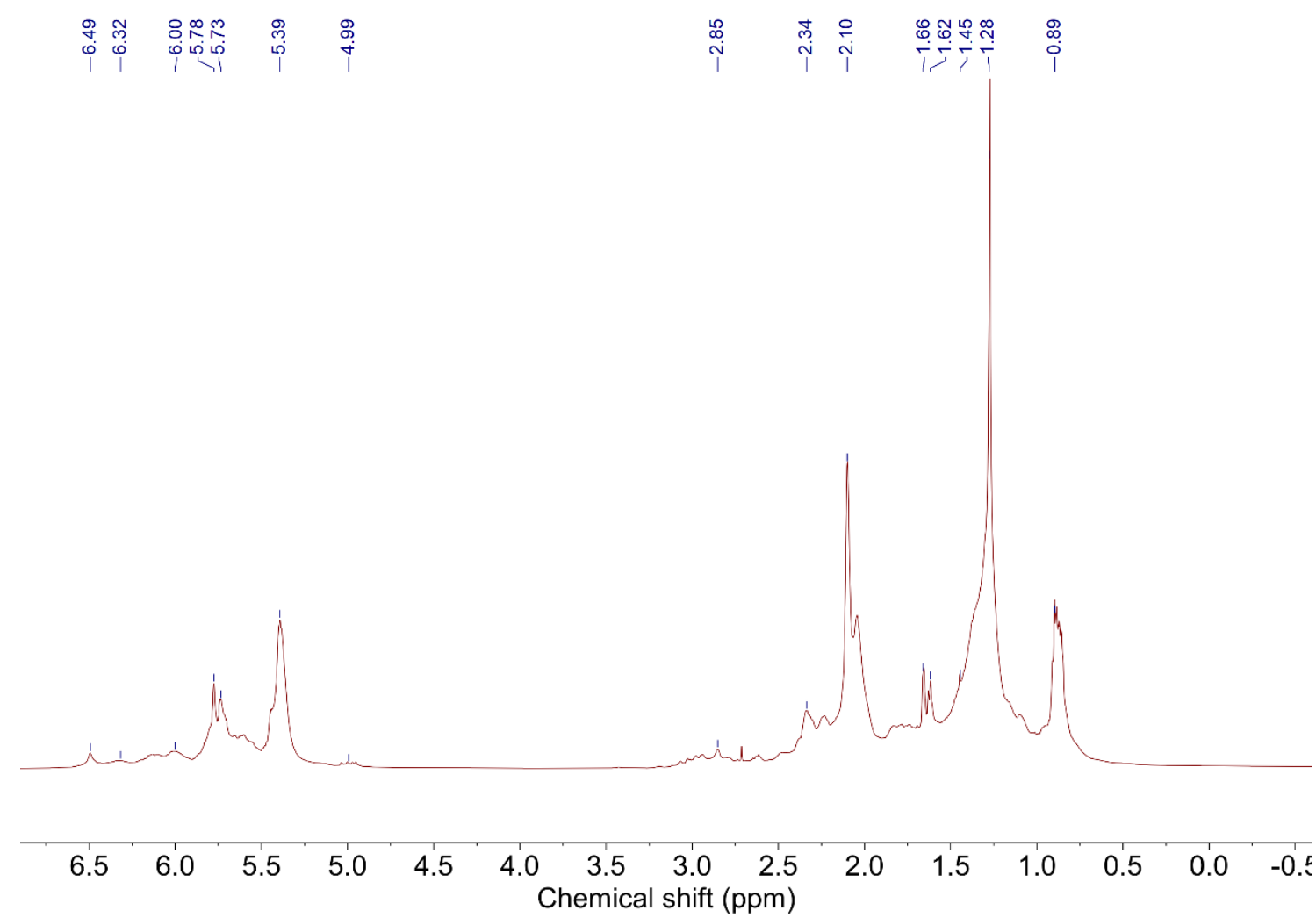

Figure S39. ${ }^{1}$ H-NMR spectrum for degraded PB (Table 1. Entry 10)

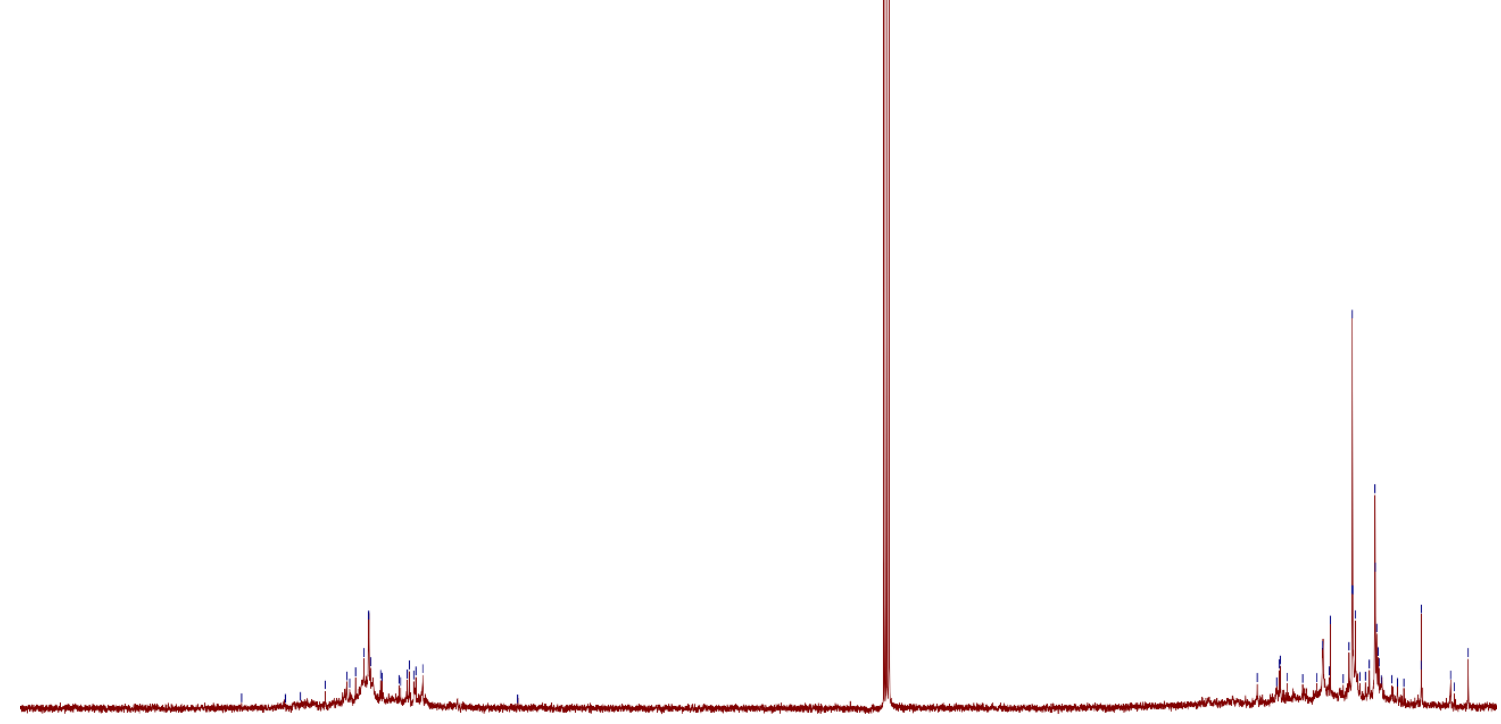

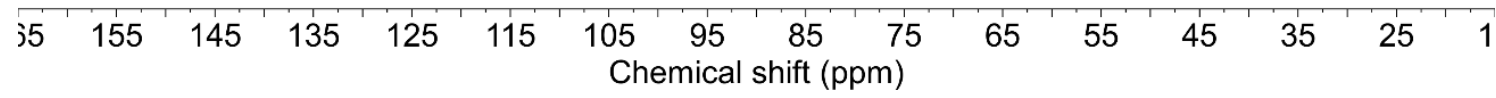

Figure S40. ${ }^{13} \mathrm{C}-\mathrm{NMR}$ spectrum for degraded PB (Table 1. Entry 10) 


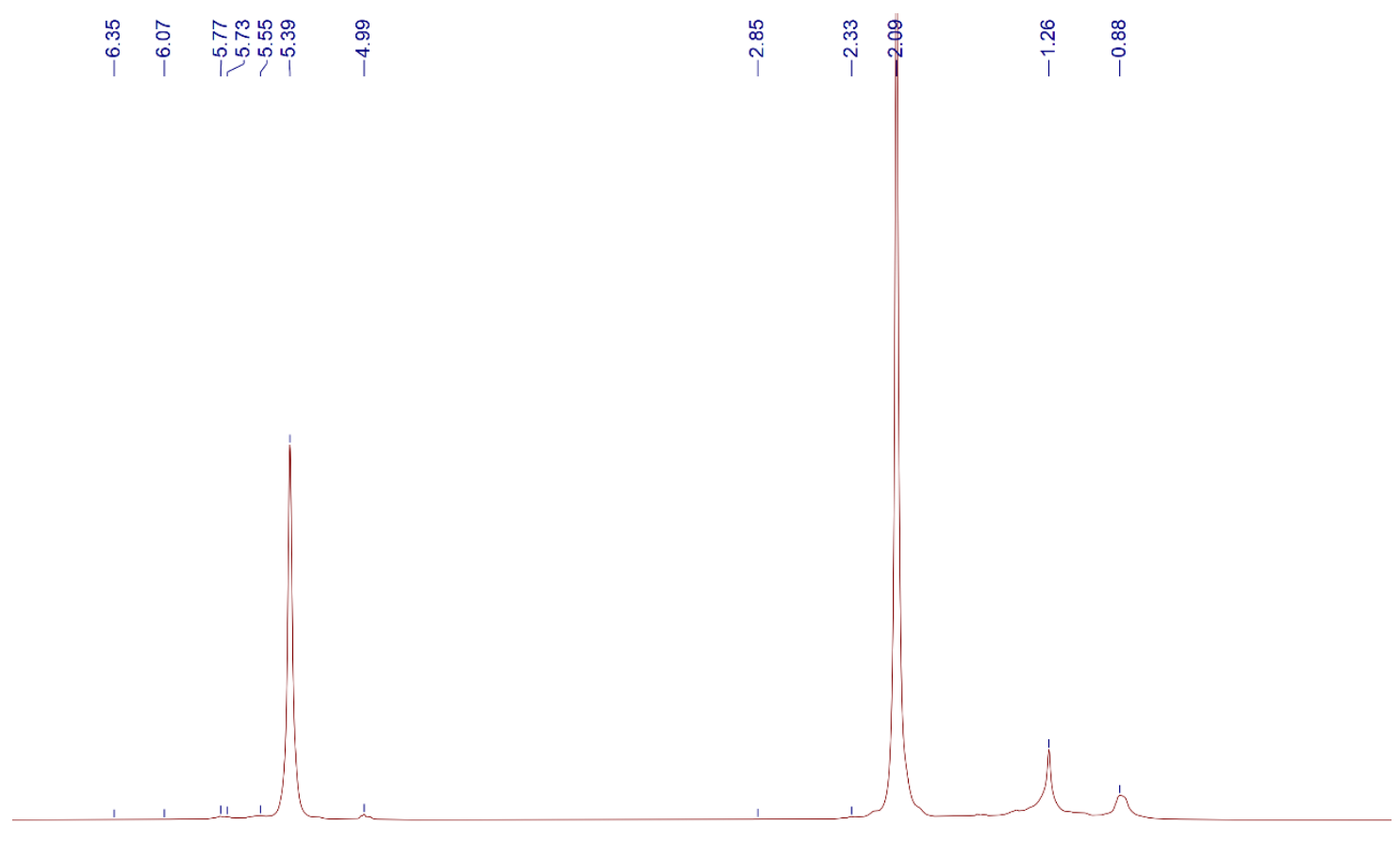

$\begin{array}{lllllllllllllll}6.5 & 6.0 & 5.5 & 5.0 & 4.5 & 4.0 & 3.5 & 3.0 & 2.5 & 2.0 & 1.5 & 1.0 & 0.5 & 0.0 & -0.5\end{array}$ Chemical shift (ppm)

Figure S41. ${ }^{1} \mathrm{H}-\mathrm{NMR}$ spectrum for degraded PB (Table 1. Entry 11)

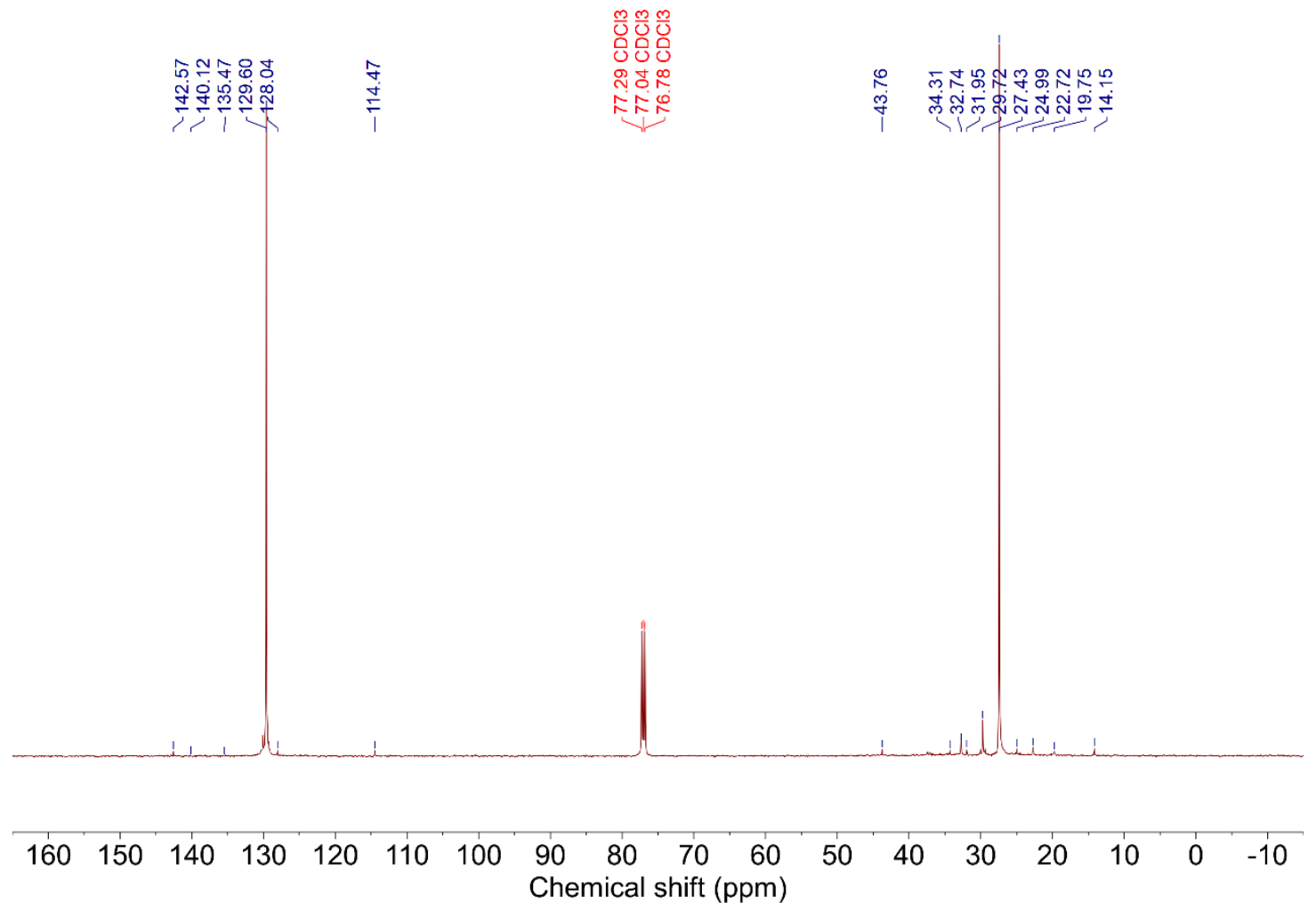

Figure S42. ${ }^{13} \mathrm{C}-\mathrm{NMR}$ spectrum for degraded PB (Table 1. Entry 11) 


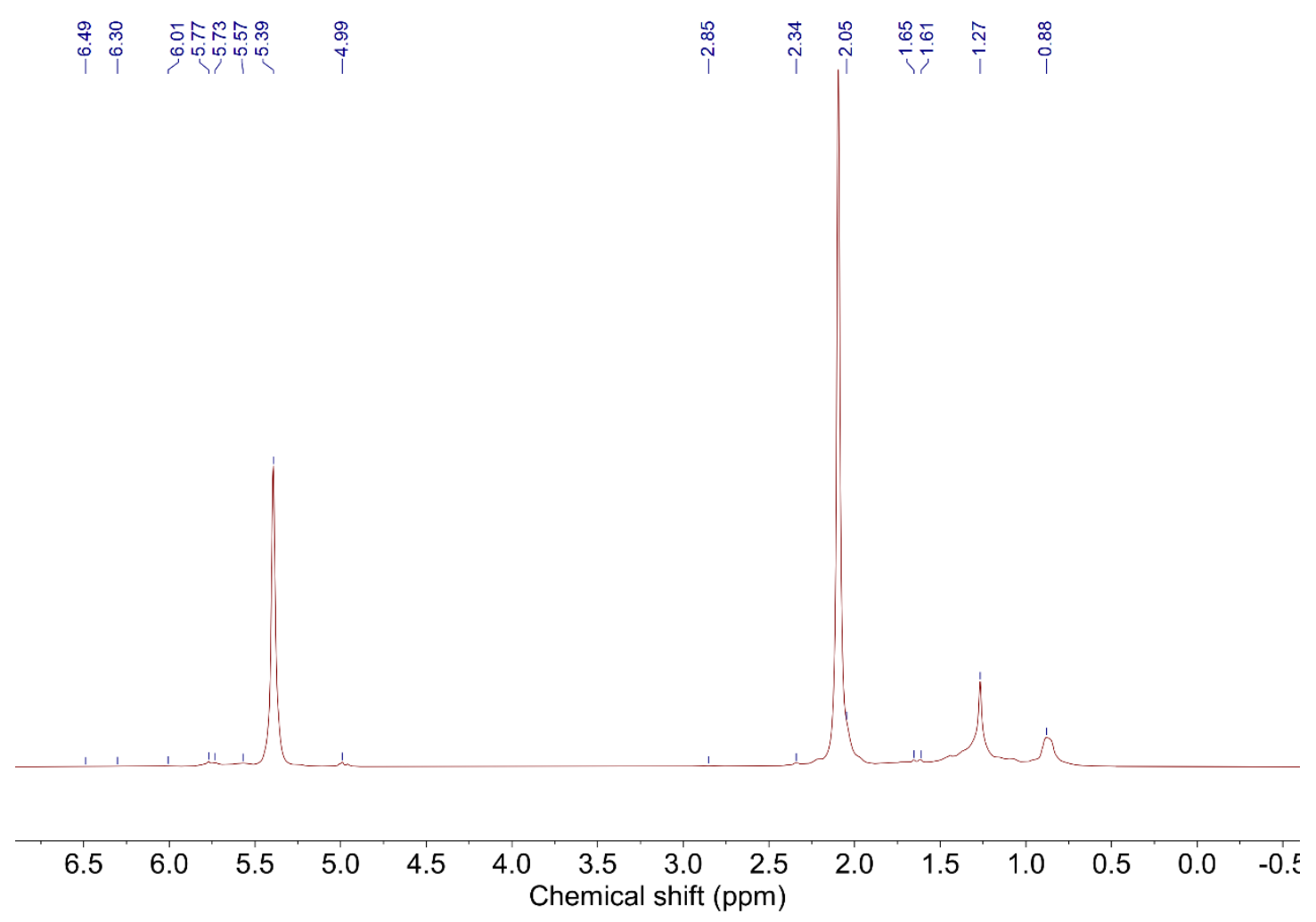

Figure S43. ${ }^{1} \mathrm{H}-\mathrm{NMR}$ spectrum for degraded PB (Table 1. Entry 12)

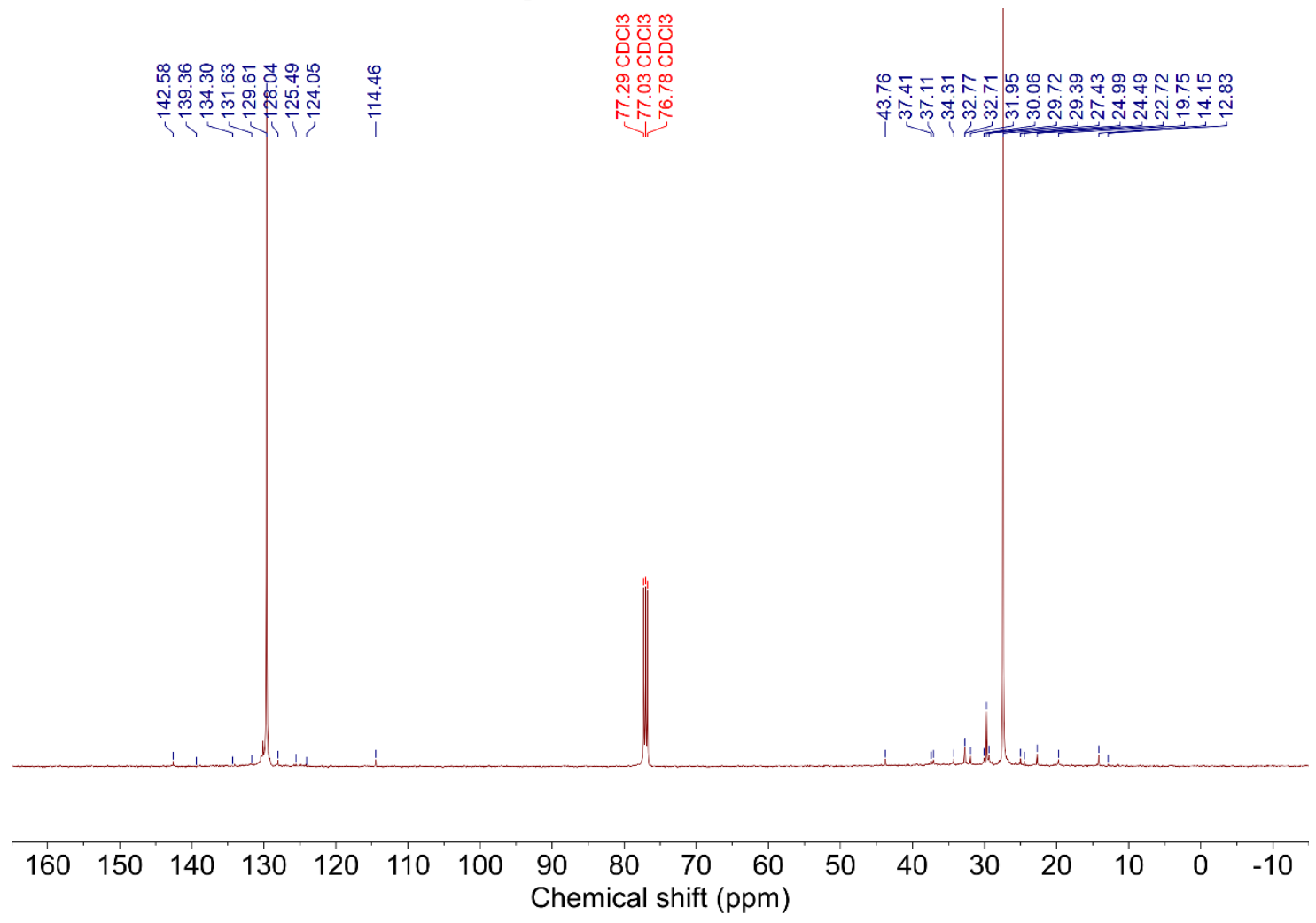

Figure S44. ${ }^{13}$ C-NMR spectrum for degraded PB (Table 1. Entry 12) 


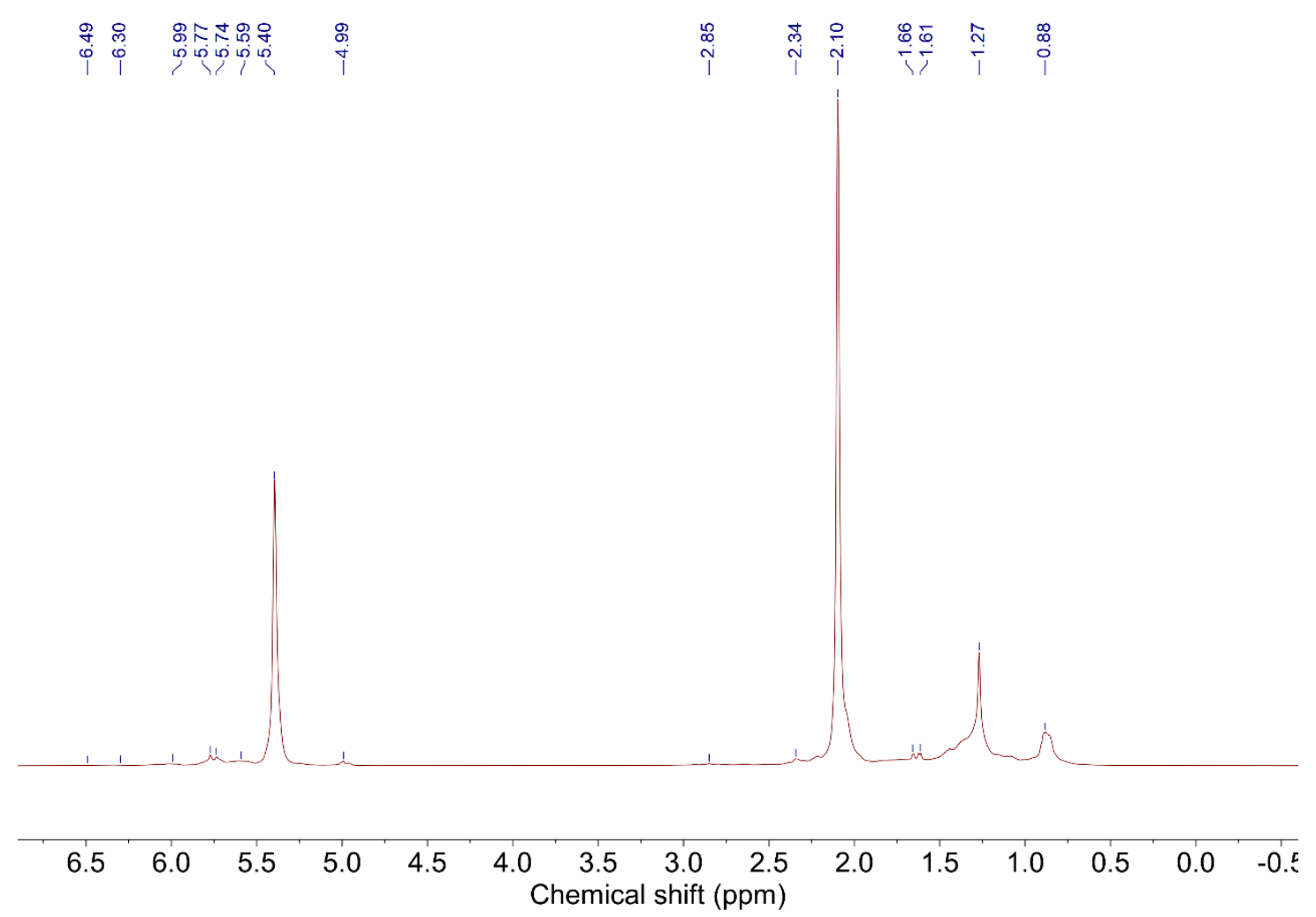

Figure S45. ${ }^{1} \mathrm{H}-\mathrm{NMR}$ spectrum for degraded PB (Table 1. Entry 13)

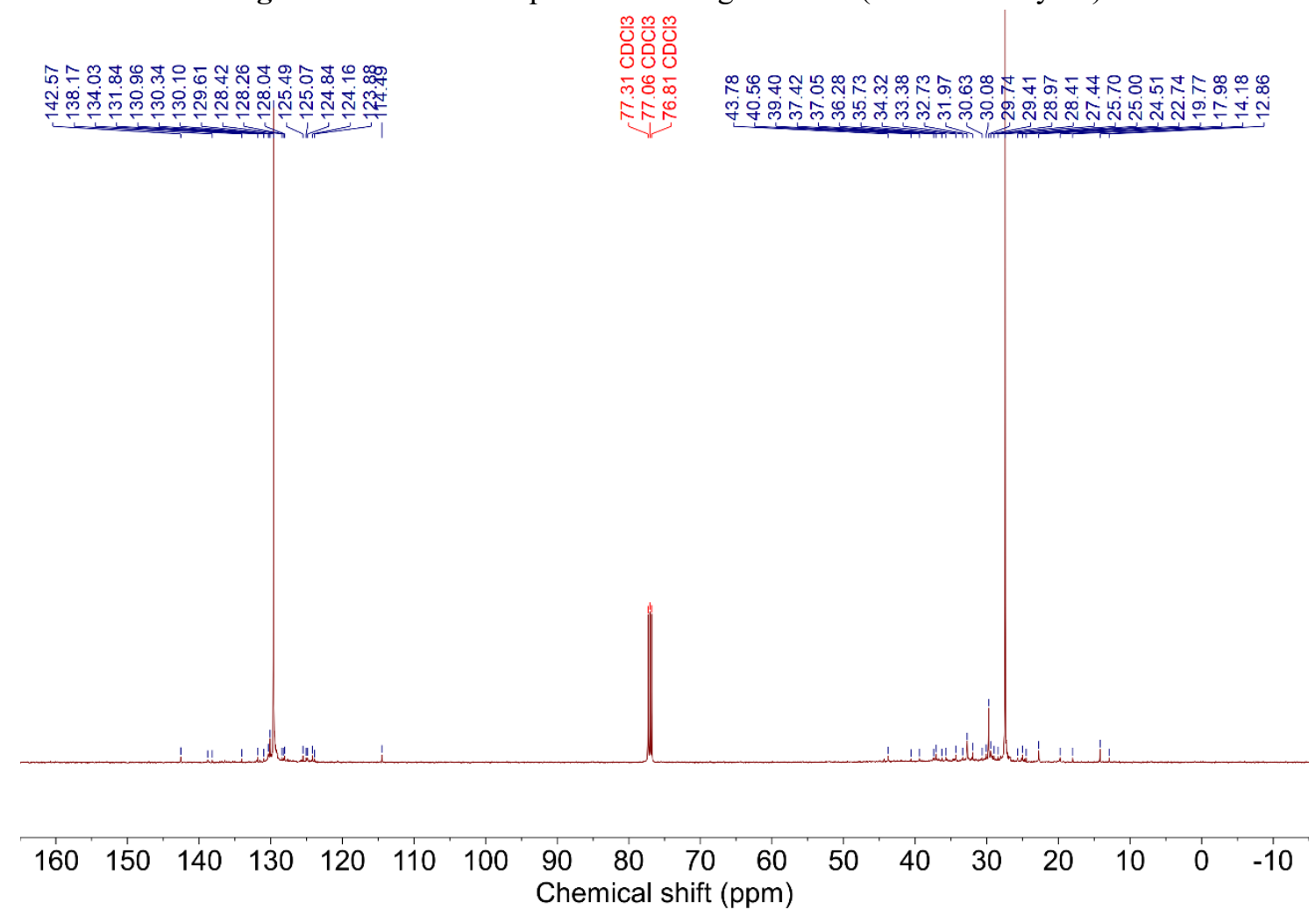

Figure S46. ${ }^{13} \mathrm{C}-\mathrm{NMR}$ spectrum for degraded PB (Table 1. Entry 13) 


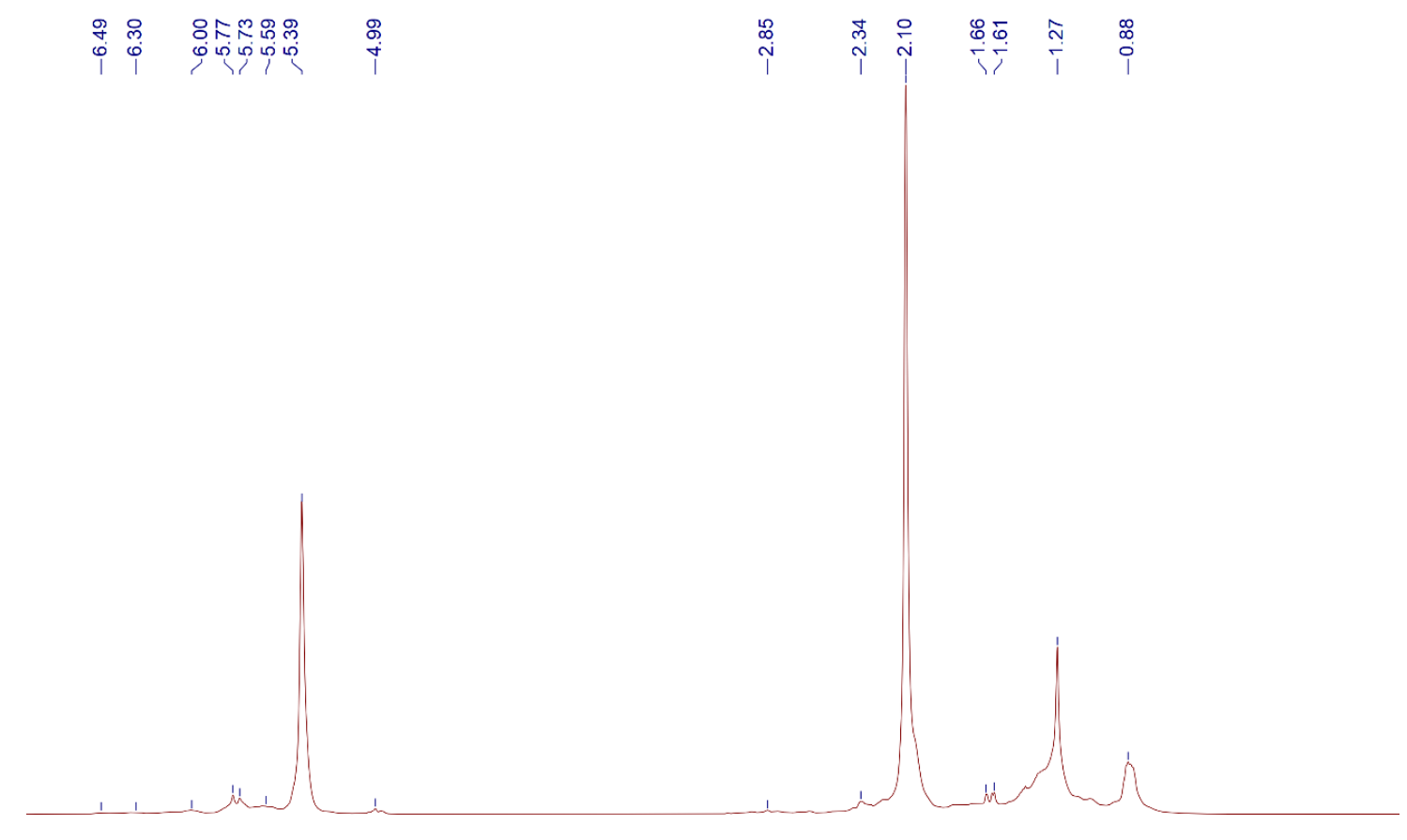

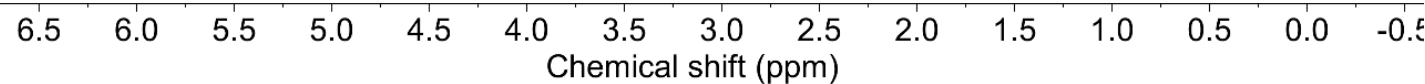

Figure S47. ${ }^{1} \mathrm{H}-\mathrm{NMR}$ spectrum for degraded PB (Table 1. Entry 14)

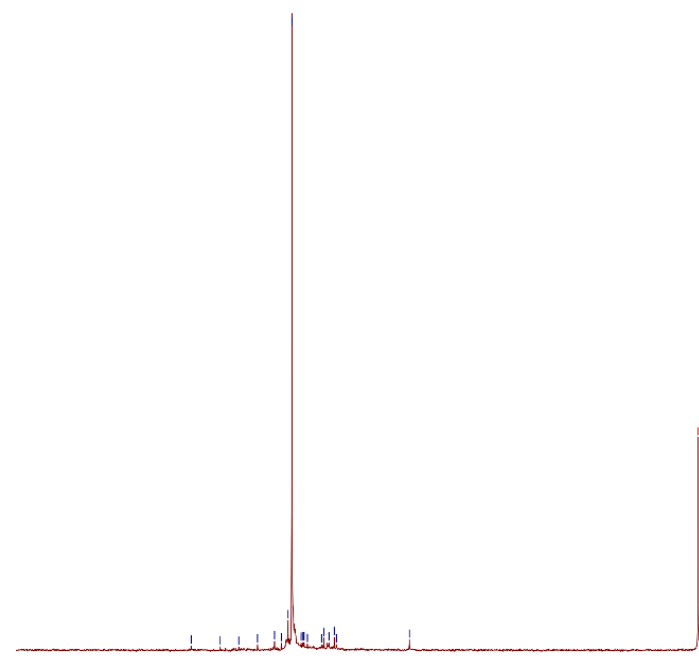

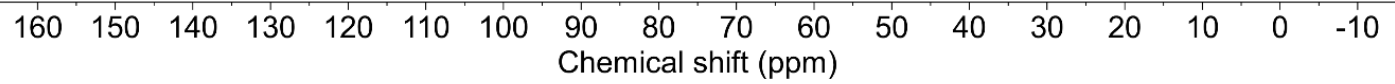

Figure S48. ${ }^{13} \mathrm{C}-\mathrm{NMR}$ spectrum for degraded PB (Table 1. Entry 14) 


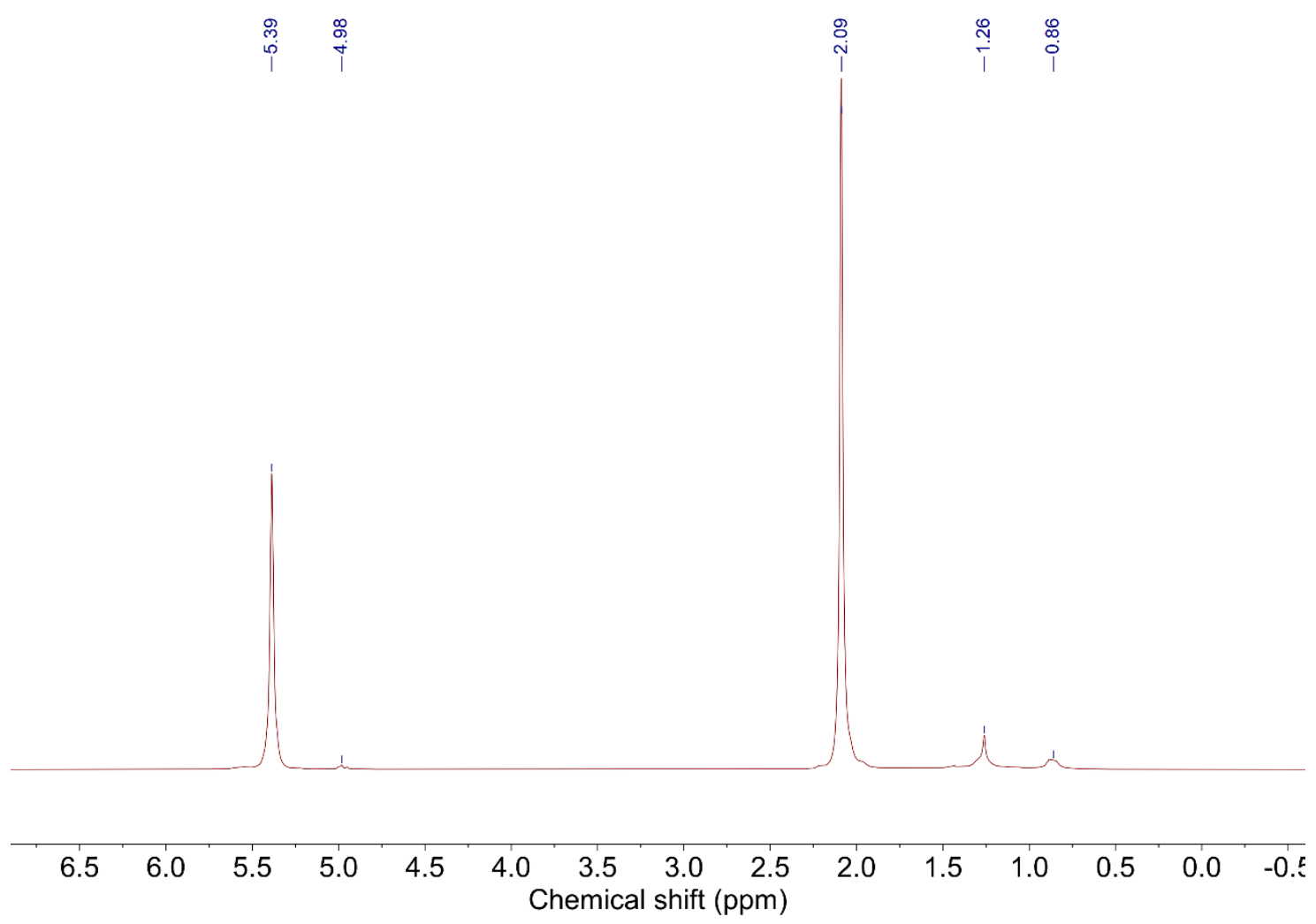

Figure S49. ${ }^{1} \mathrm{H}-\mathrm{NMR}$ spectrum for degraded PB (Table 1. Entry 15)

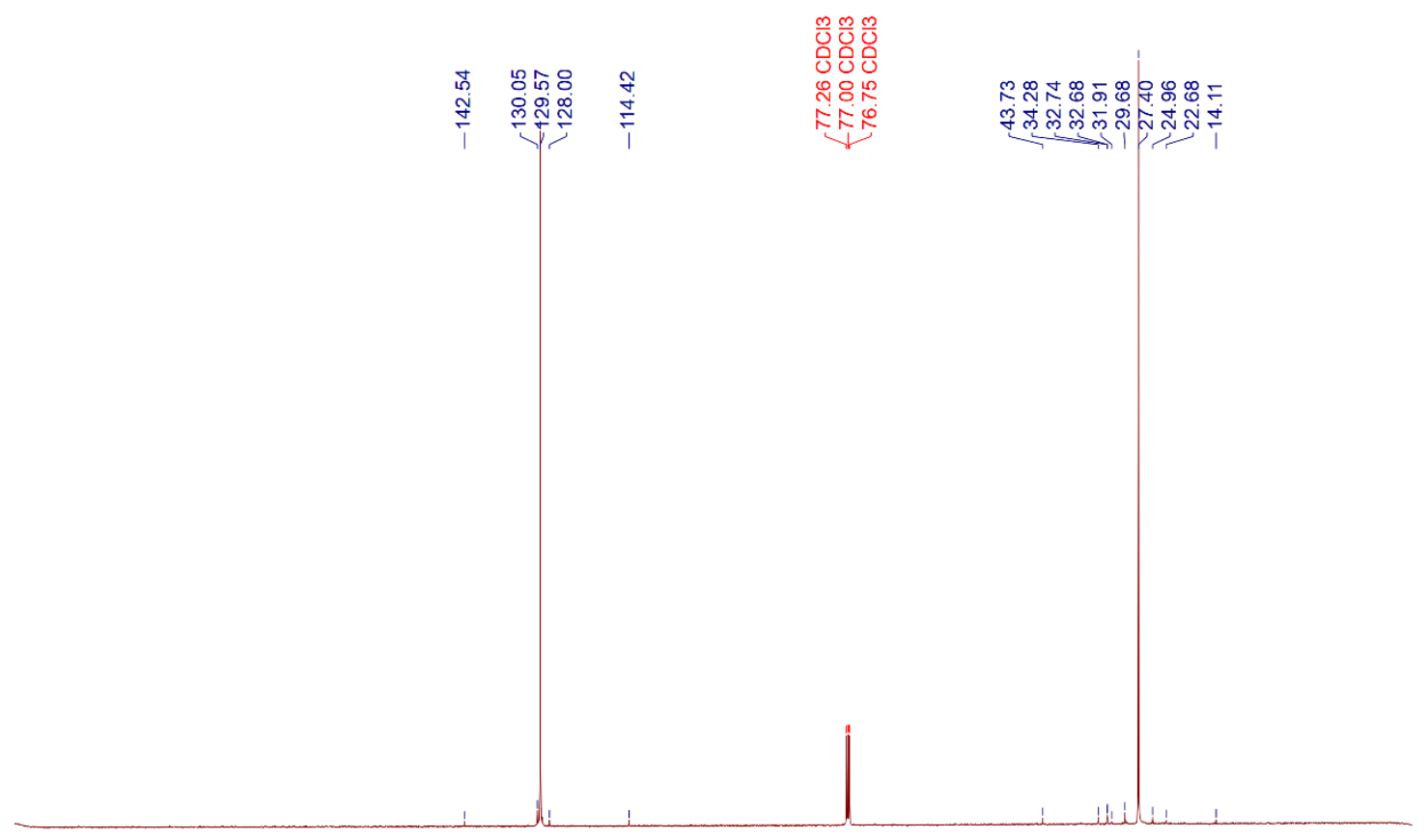

$21020019018017016015014013012011010090 \quad 8070$ 60 $50 \begin{array}{llllllll}30 & 20 & 10 & 0 & -10\end{array}$ Chemical shift (ppm)

Figure S50. ${ }^{13} \mathrm{C}-\mathrm{NMR}$ spectrum for degraded PB (Table 1. Entry 15) 


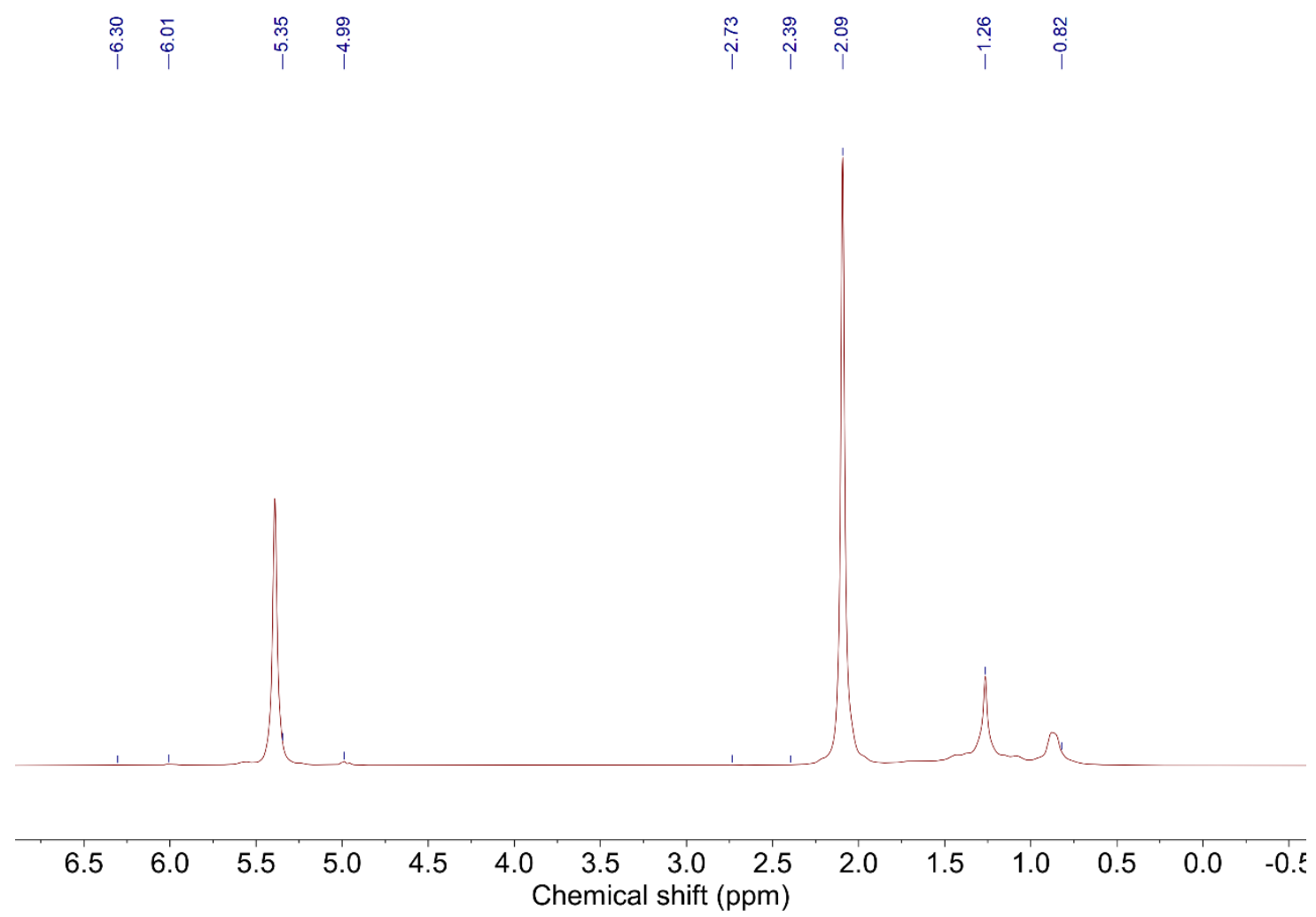

Figure S51. ${ }^{1} \mathrm{H}-\mathrm{NMR}$ spectrum for degraded PB (Table 1. Entry 16)

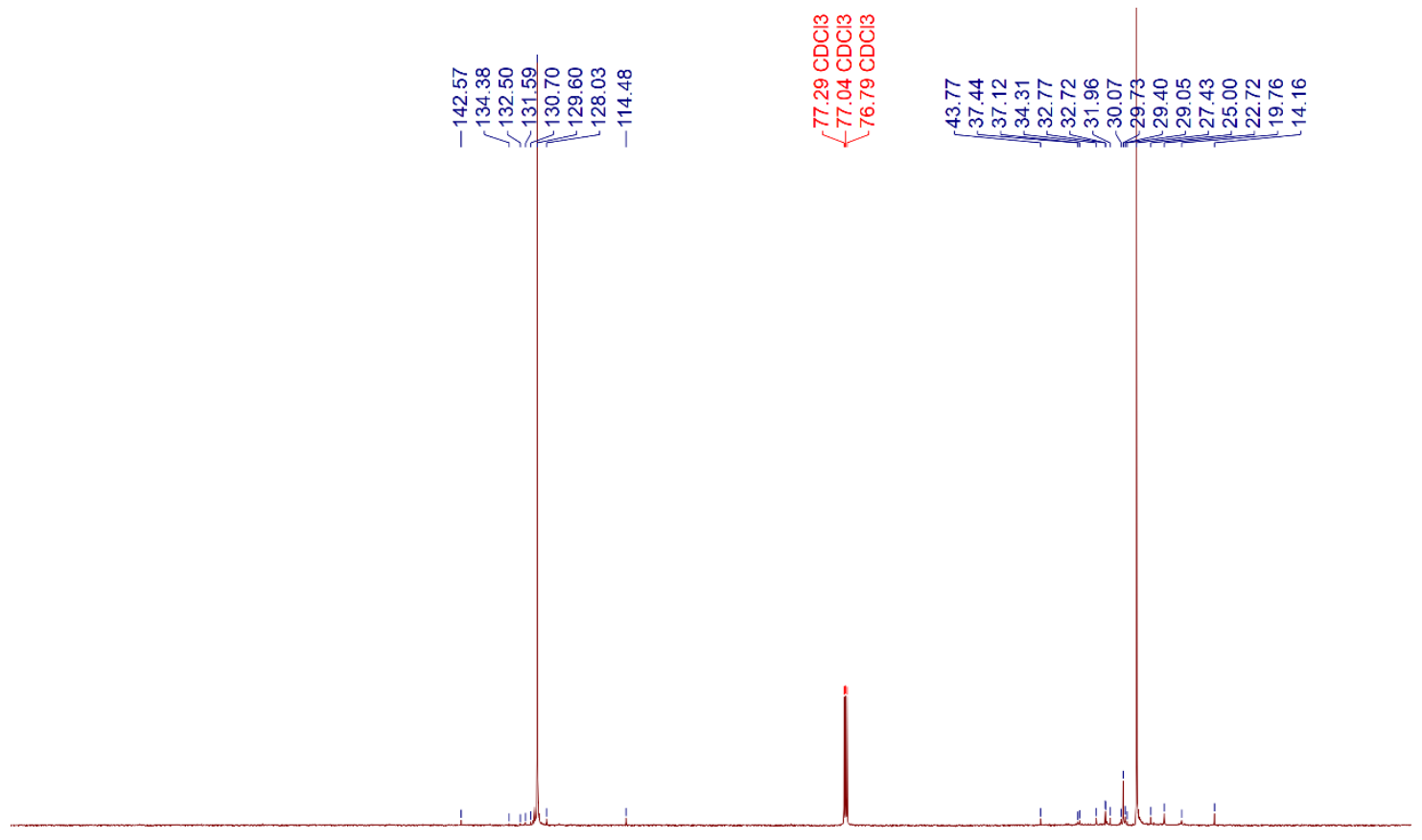

$210200190180170160150140130120110100 \quad 90 \quad 8070 \quad 60 \begin{array}{llllllll}1 & 40 & 30 & 20 & 10 & 0 & -10\end{array}$ Chemical shift (ppm)

Figure S52. ${ }^{13} \mathrm{C}-\mathrm{NMR}$ spectrum for degraded PB (Table 1. Entry 16) 


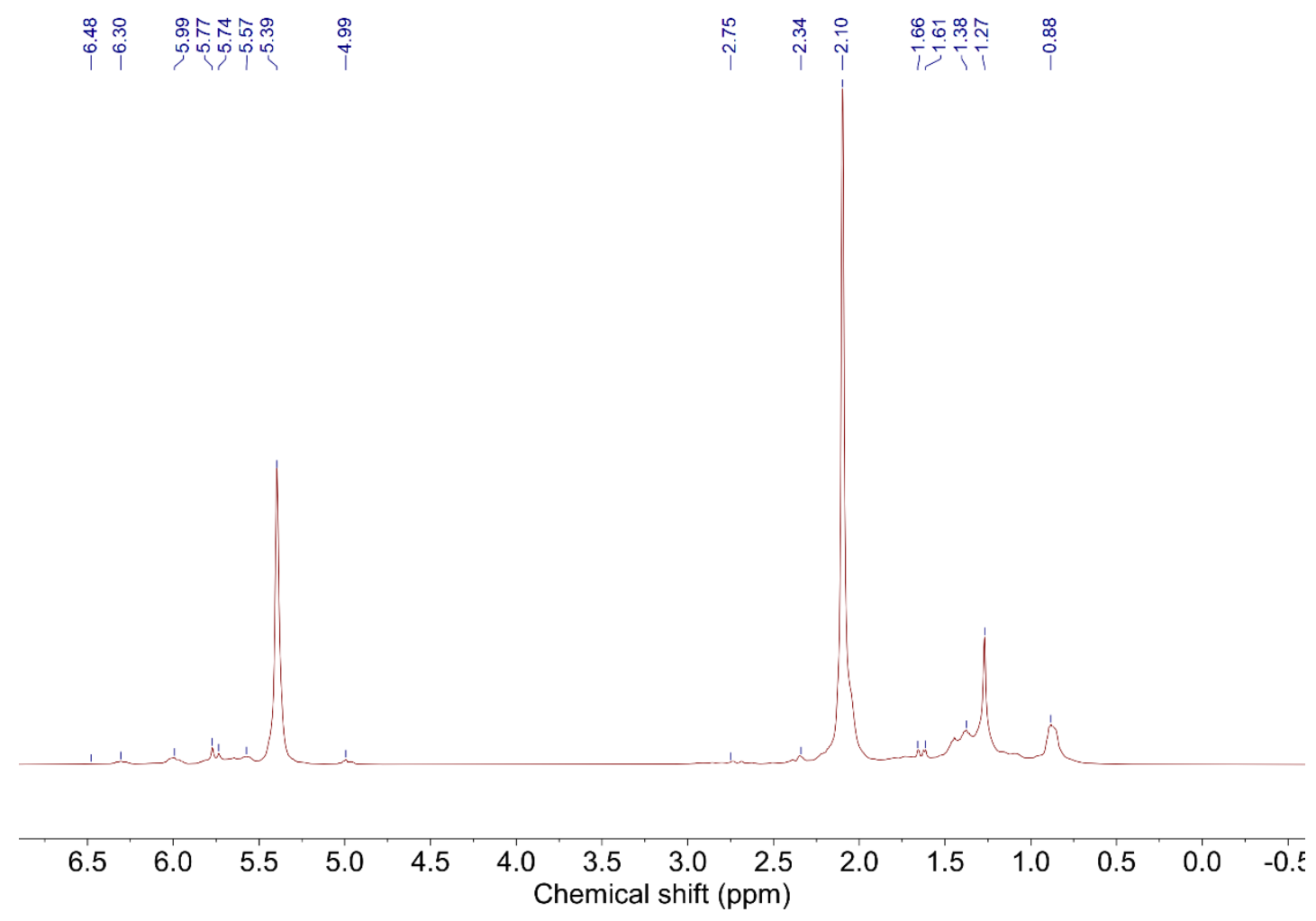

Figure S53. ${ }^{1} \mathrm{H}-\mathrm{NMR}$ spectrum for degraded PB (Table 1. Entry 17)

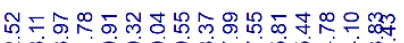

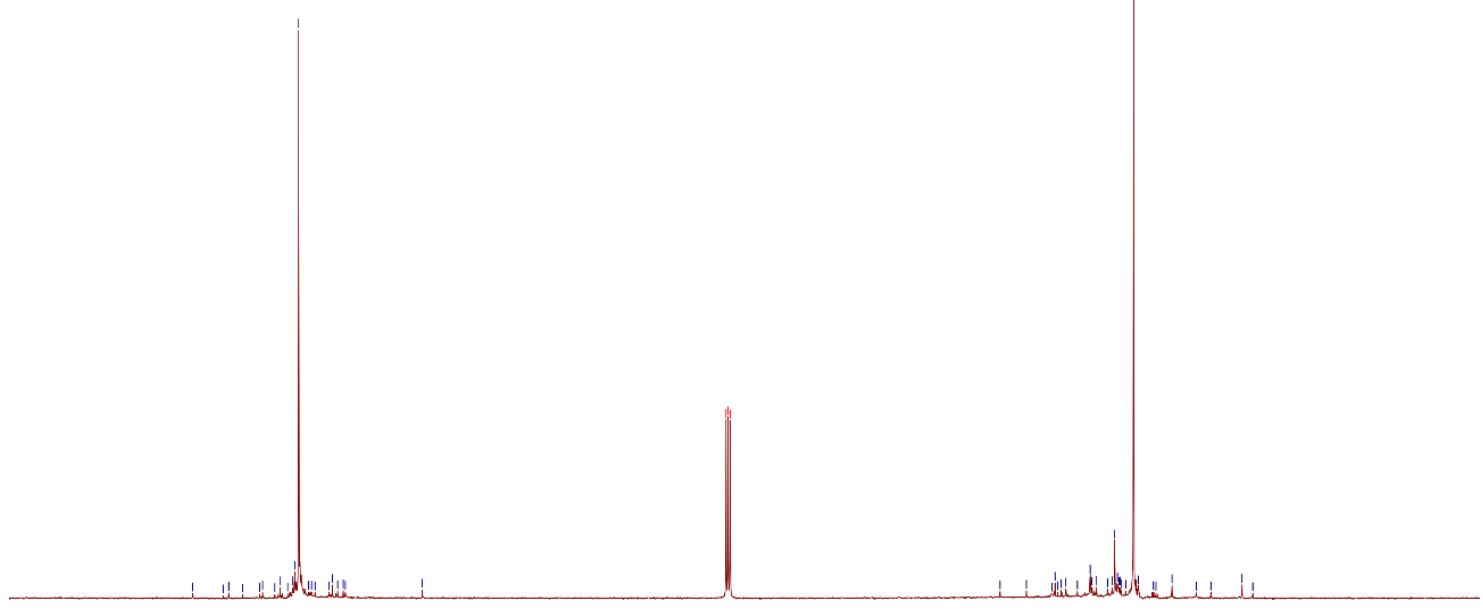

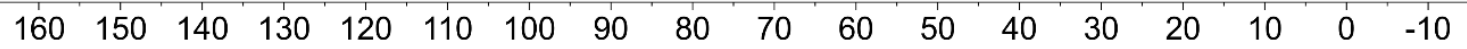
Chemical shift (ppm)

Figure S54. ${ }^{13}$ C-NMR spectrum for degraded PB (Table 1. Entry 17) 


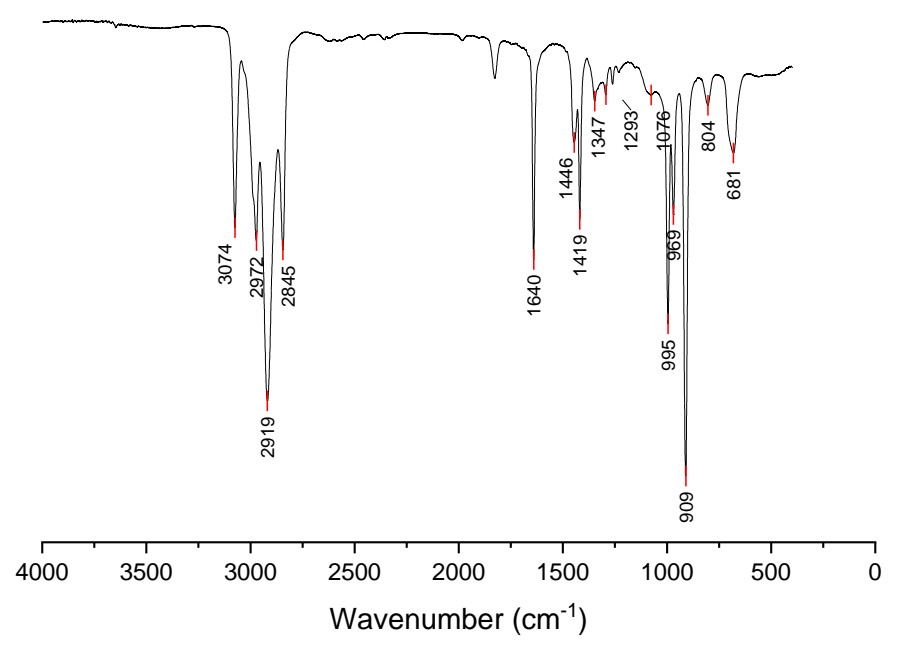

Figure S55. FTIR spectrum of HVPB (Table 2. Entry 3)

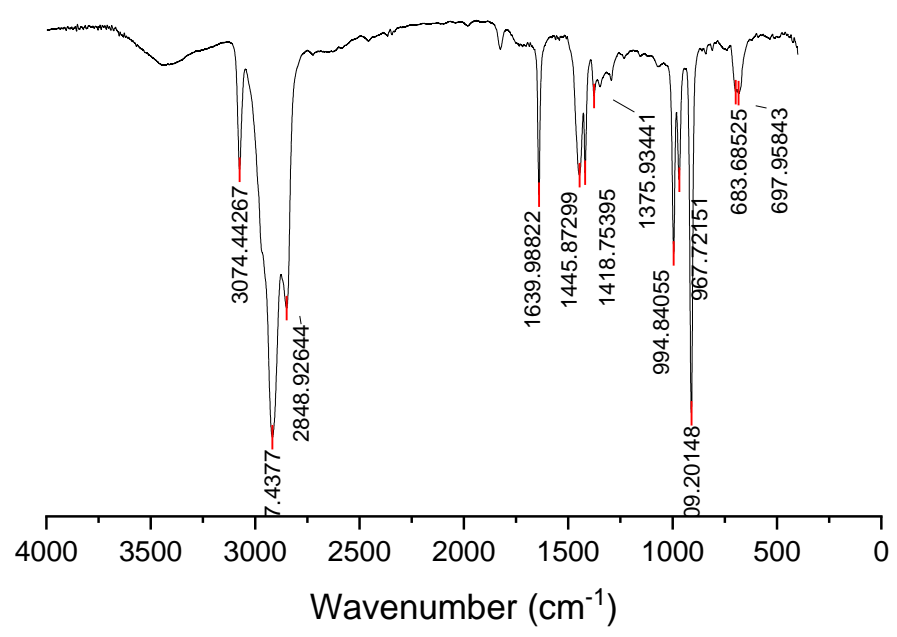

Figure S56. FTIR spectrum of HVPB after degradation (Table 2. Entry 4) 


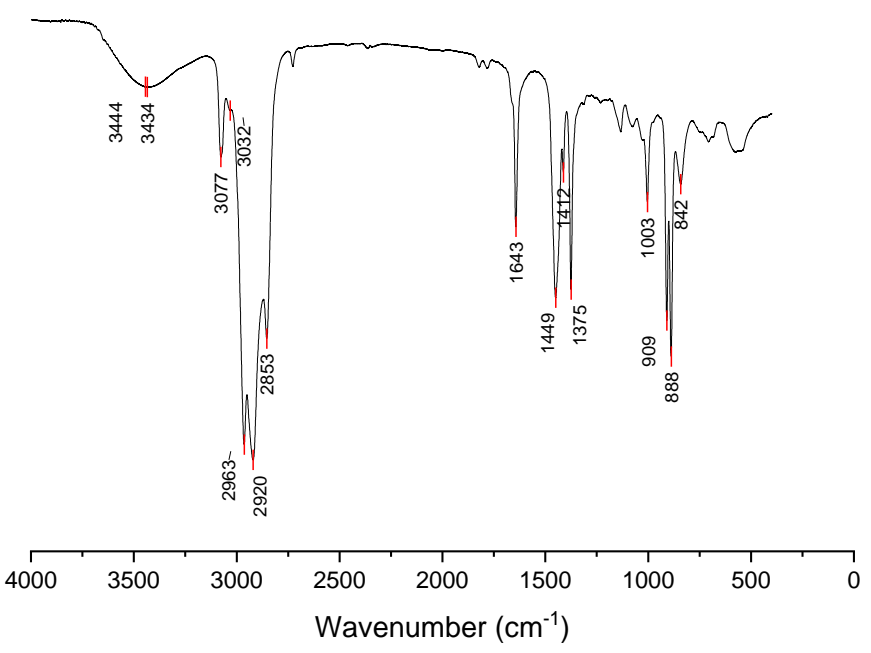

Figure S57. FTIR spectrum of PI after degradation (Table 2. Entry 5)

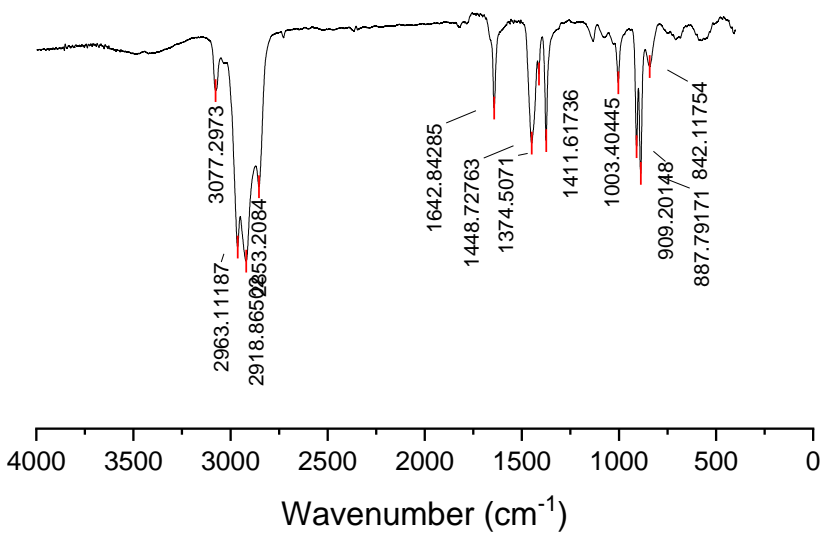

Figure S58. FTIR spectrum of PI after degradation (Table 2. Entry 6) 


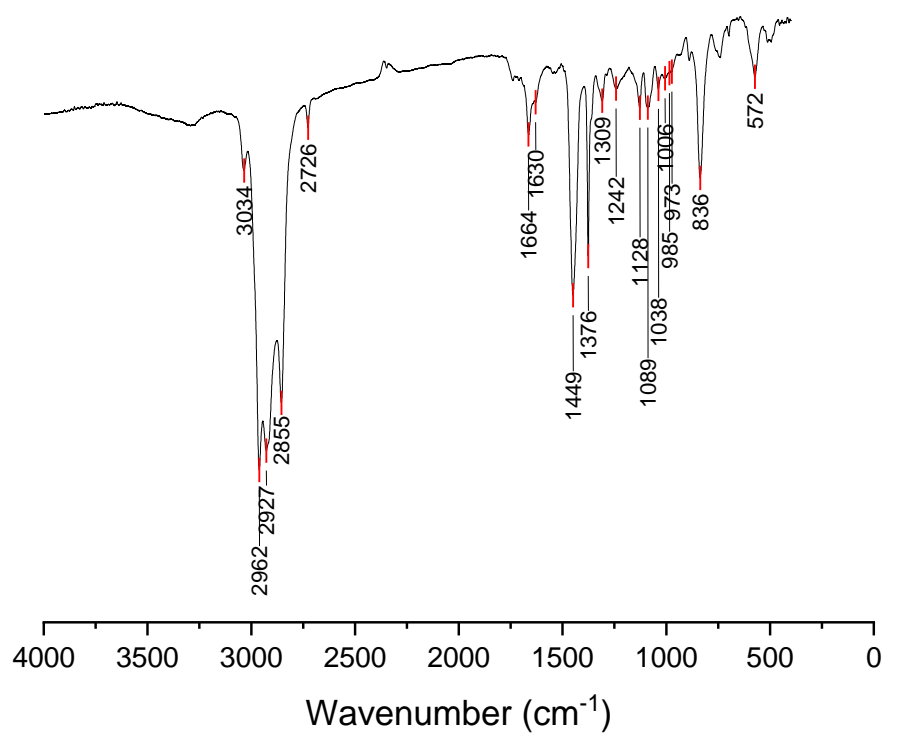

Figure S59. FTIR spectrum of NR (Table 2. Entry 7)

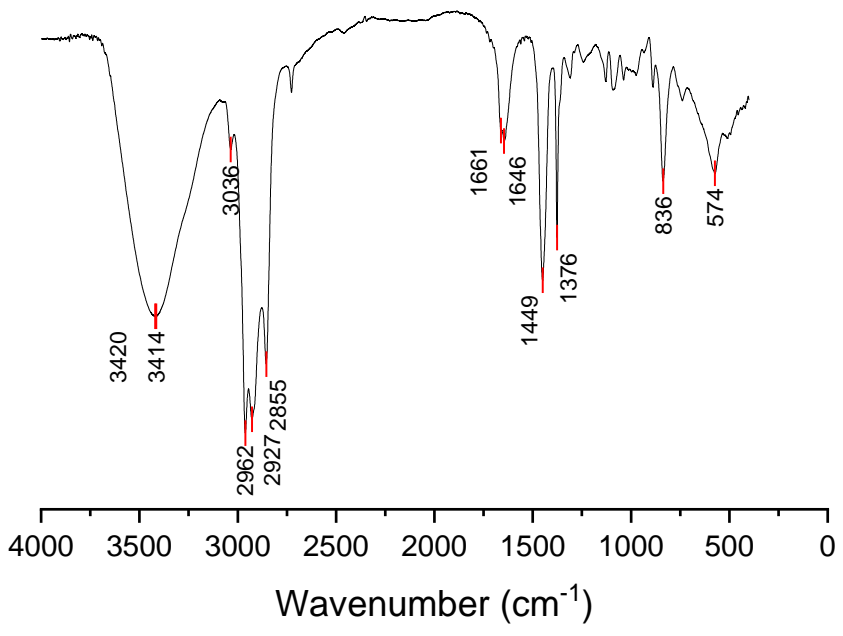

Figure S60. FTIR spectrum of NR after degradation (Table 2. Entry 8) 


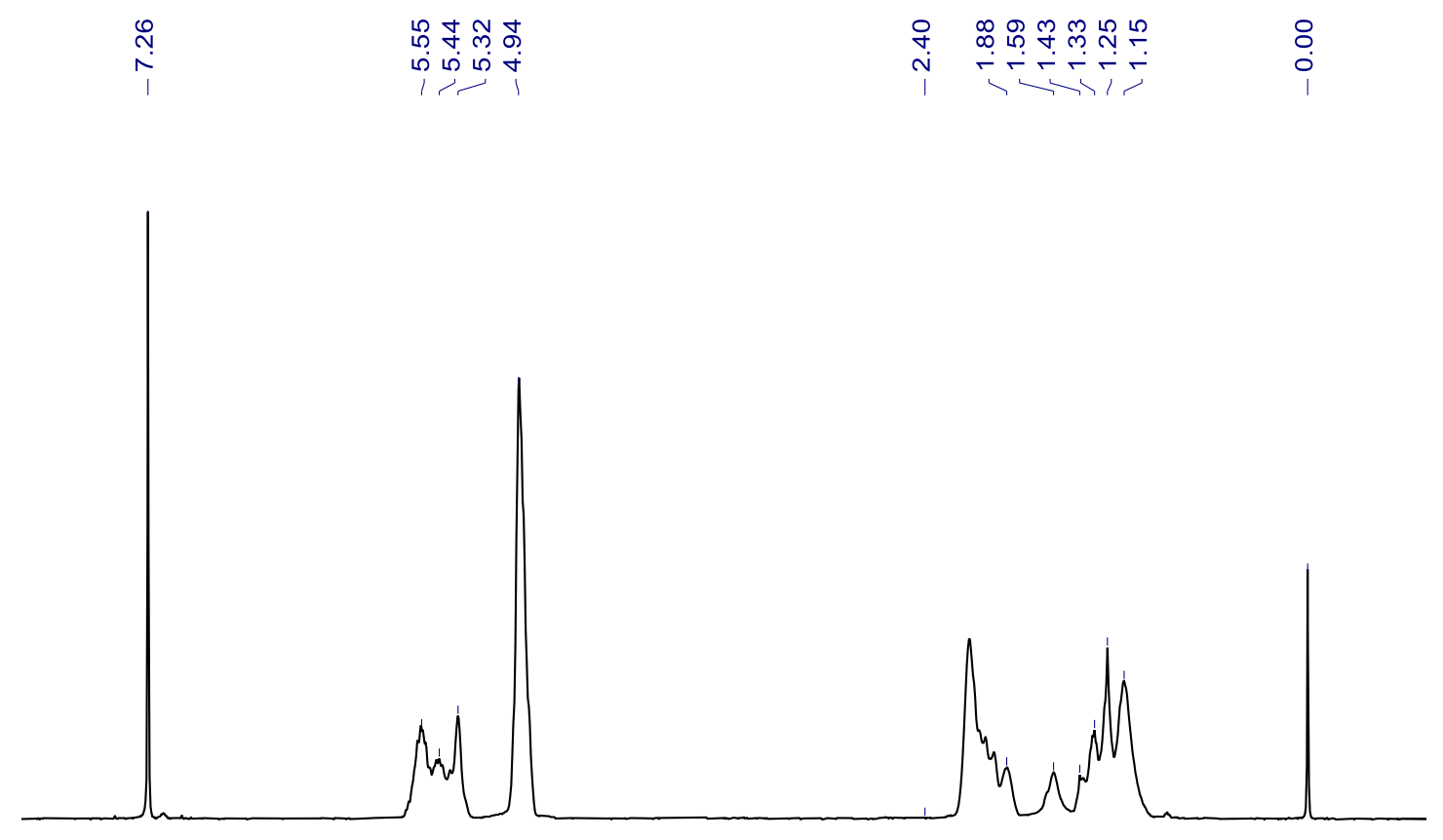

\begin{tabular}{lllllllllllllllllllll}
\hline 3.0 & 7.5 & 7.0 & 6.5 & 6.0 & 5.5 & 5.0 & 4.5 & 4.0 & 3.5 & 3.0 & 2.5 & 2.0 & 1.5 & 1.0 & 0.5 & 0.0 & -0.5
\end{tabular} Chemical shift (ppm)

Figure S61. ${ }^{1} \mathrm{H}-\mathrm{NMR}$ spectrum of HVPB

\begin{tabular}{|c|c|c|}
\hline 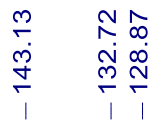 & $\underset{⿱ 亠}{\stackrel{+}{+}}$ & 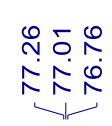 \\
\hline
\end{tabular}

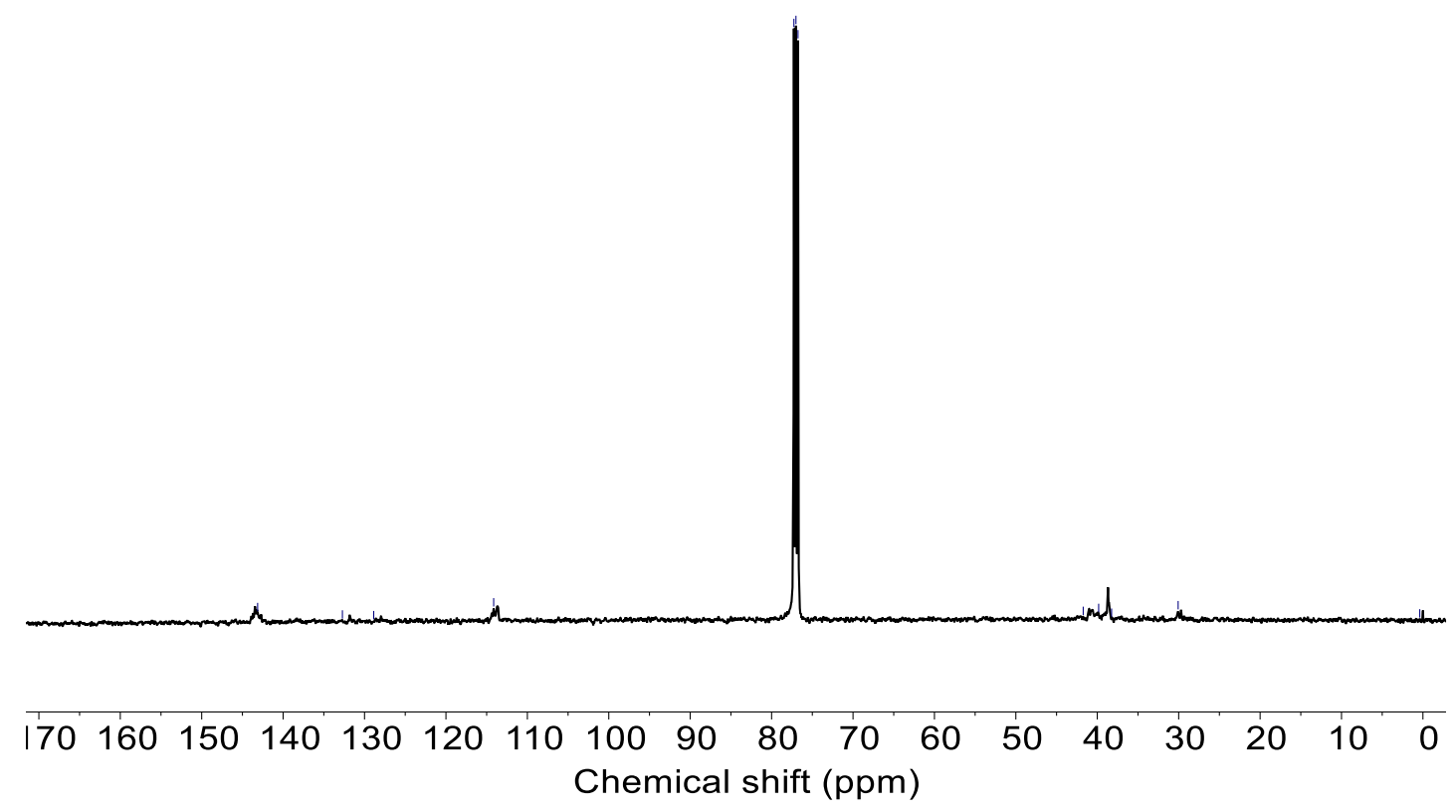

Figure S62. ${ }^{13} \mathrm{C}-\mathrm{NMR}$ spectrum of HVPB 


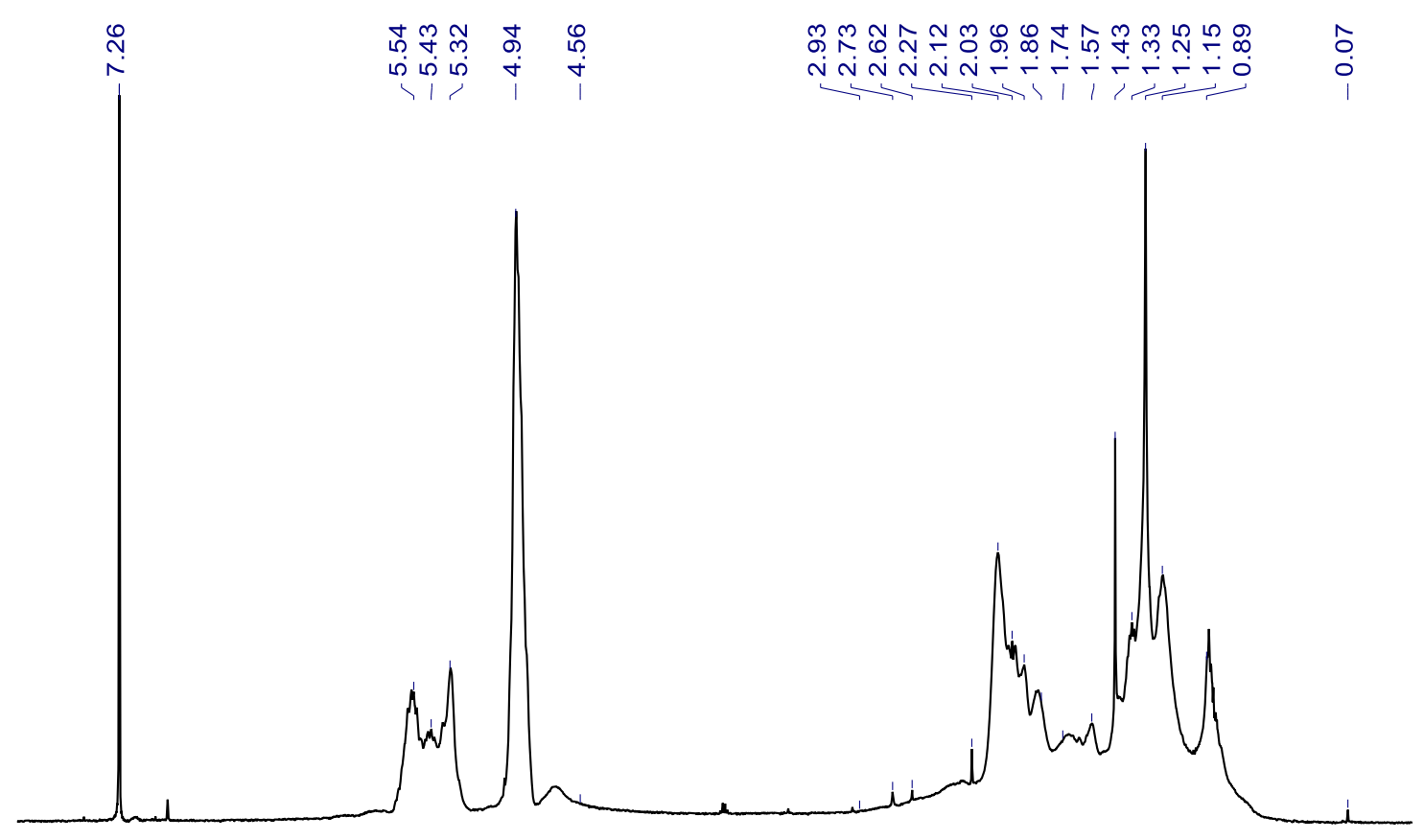

$\begin{array}{cccccccccccccccc}7.5 & 7.0 & 6.5 & 6.0 & 5.5 & 5.0 & \begin{array}{c}4.5 \\ \text { Chemical shift (ppm) }\end{array} & 4.0 & 3.5 & 3.0 & 2.5 & 2.0 & 1.5 & 1.0 & 0.5 & 0.0\end{array}$

Figure S63. 1H-NMR spectrum of HVPB after degradation

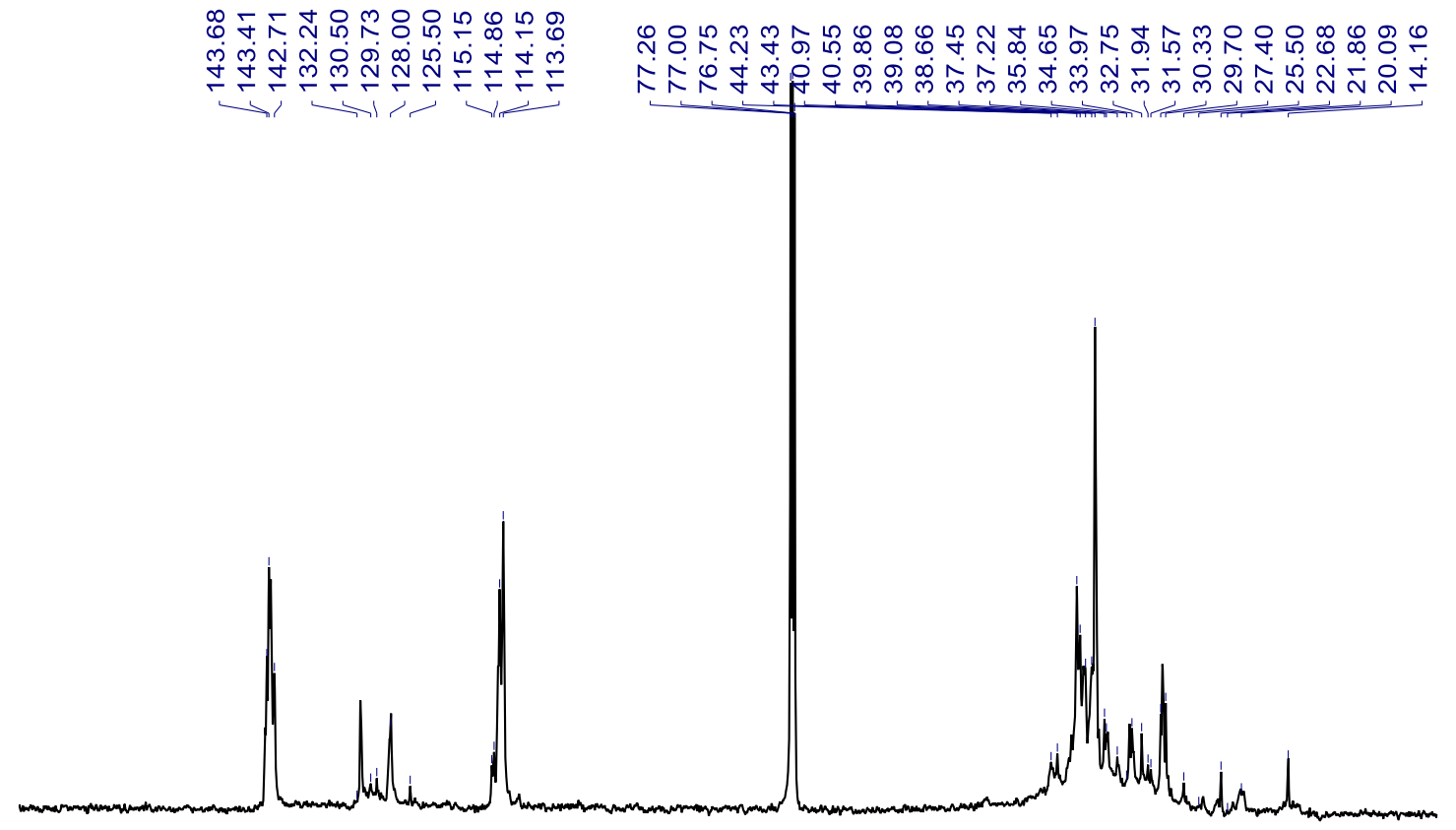

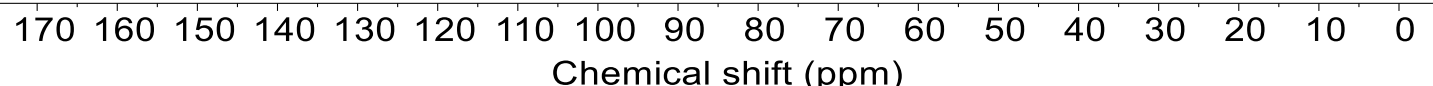

Figure S64. ${ }^{13} \mathrm{C}-\mathrm{NMR}$ spectrum of HVPB after degradation 


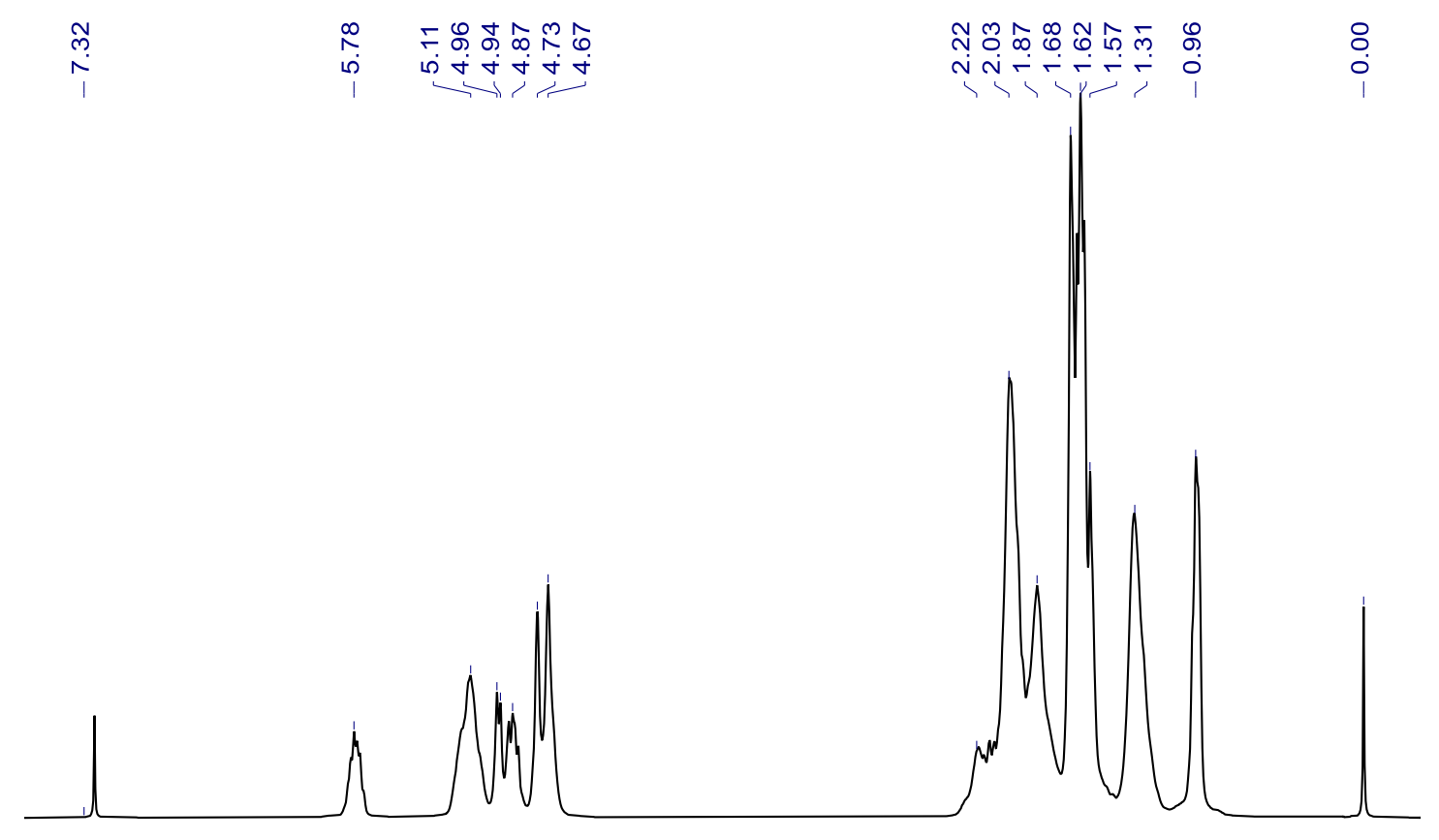

\section{$\begin{array}{lllllllllllllllll}7.5 & 7.0 & 6.5 & 6.0 & 5.5 & 5.0 & \begin{array}{l}4.5 \\ \text { Chemical shift }(\mathrm{ppm})\end{array} & 4.0 & 3.5 & 3.0 & 2.5 & 2.0 & 1.5 & 1.0 & 0.5 & 0.0\end{array}$}

Figure S65. ${ }^{1} \mathrm{H}-\mathrm{NMR}$ spectrum of PI
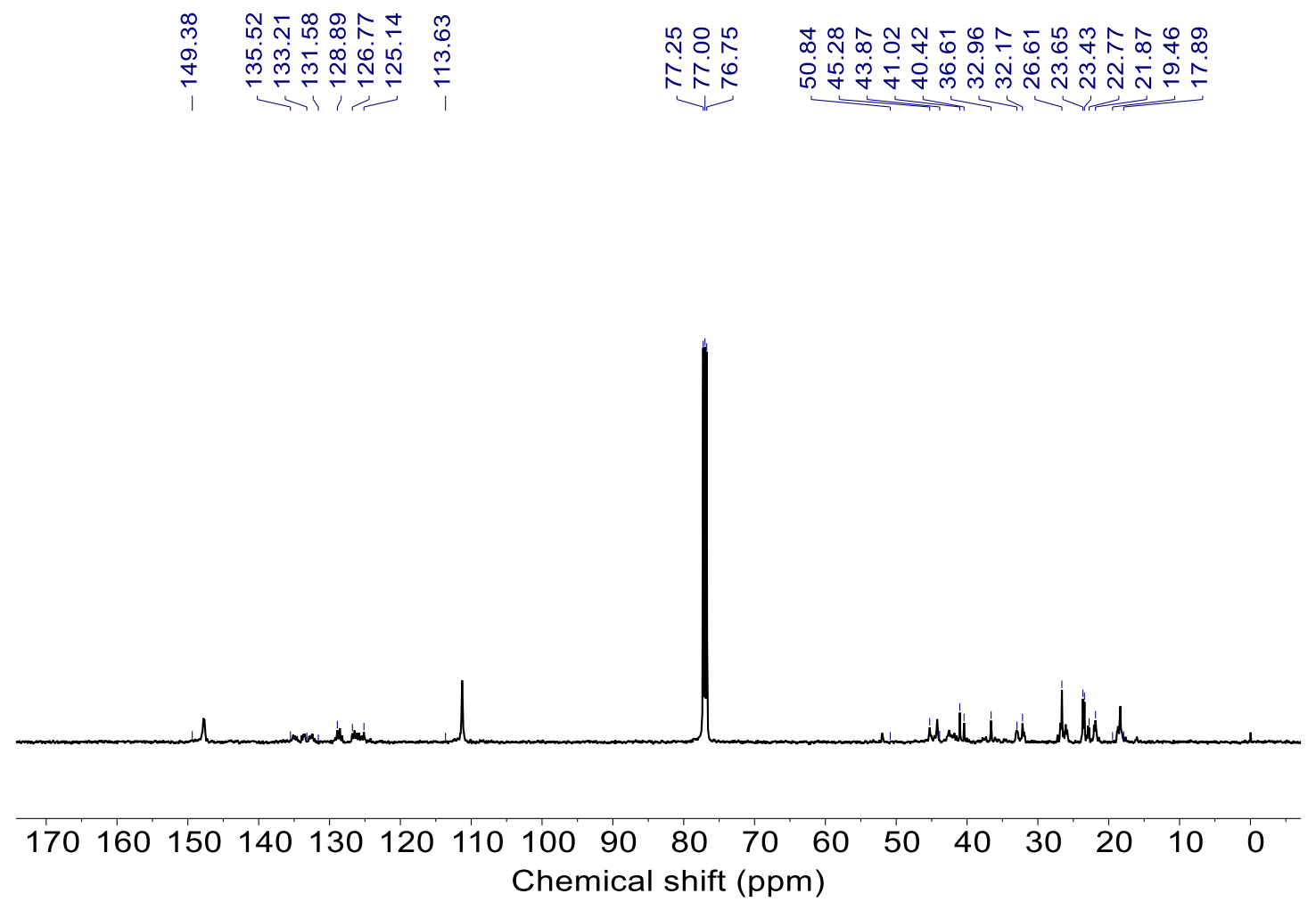

Figure S66. ${ }^{13} \mathrm{C}$-NMR spectrum of PI 

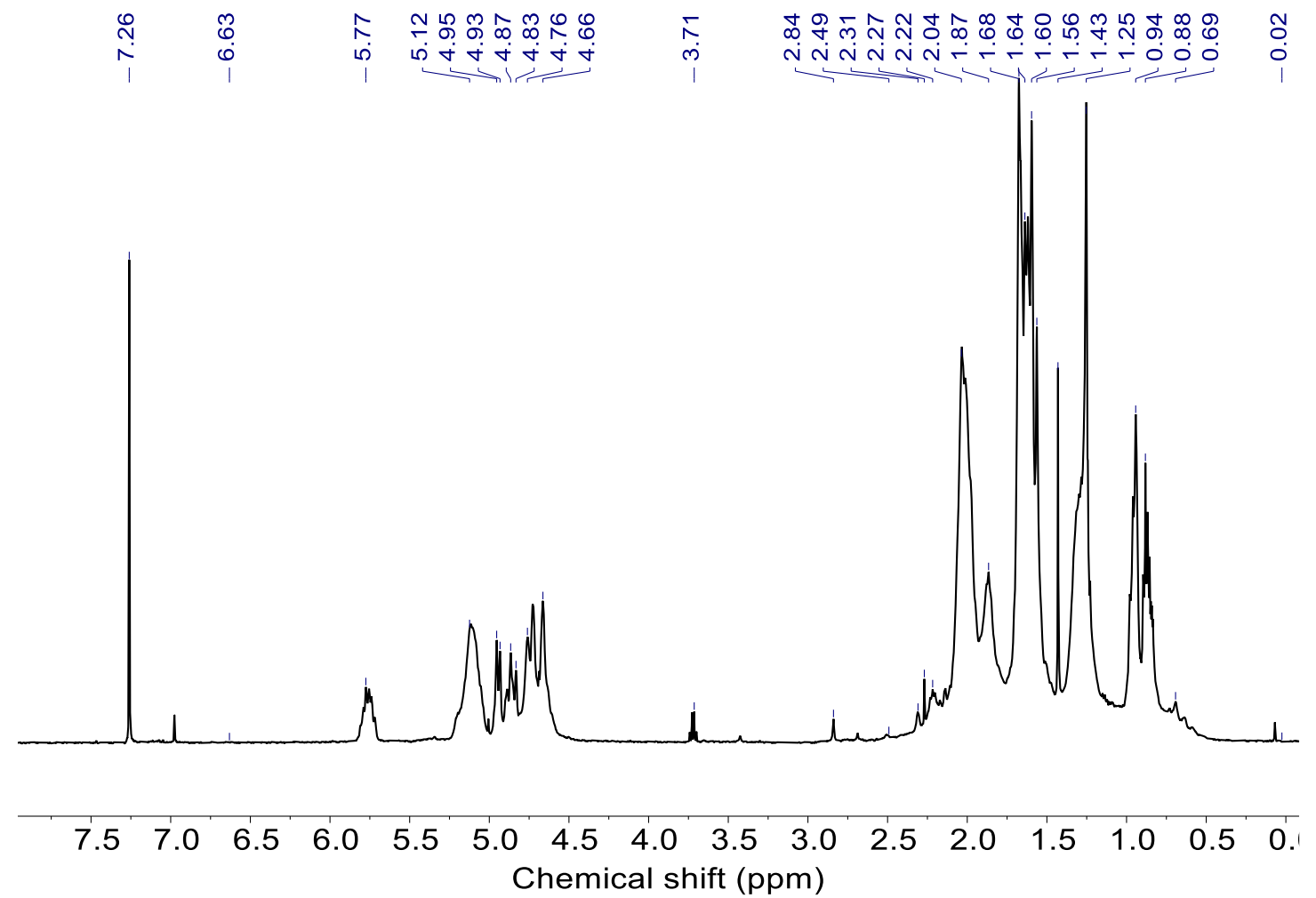

Figure S67. ${ }^{1} \mathrm{H}-\mathrm{NMR}$ spectrum of PI after degradation
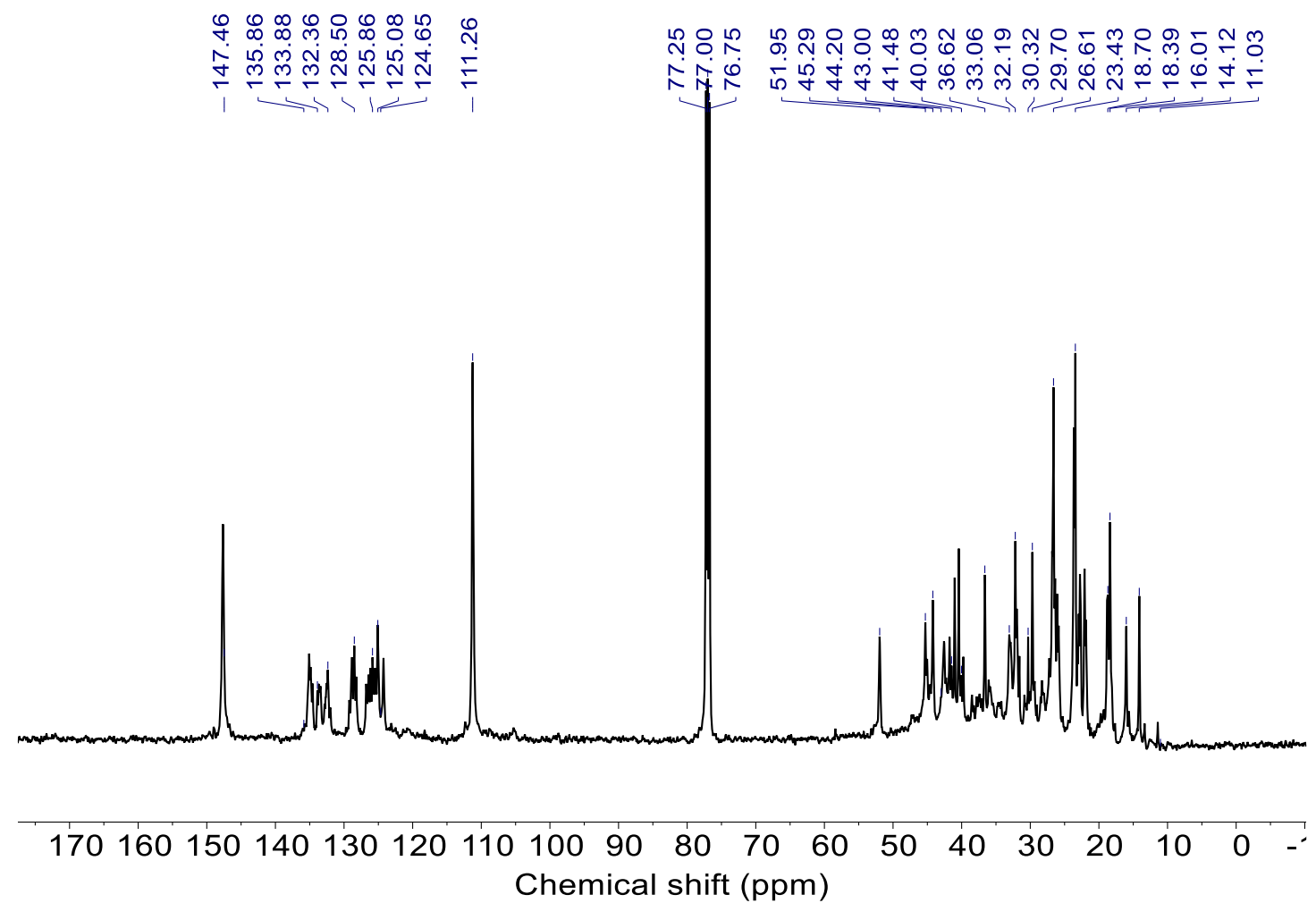

Figure S68. ${ }^{13} \mathrm{C}-\mathrm{NMR}$ spectrum of PI after degradation 


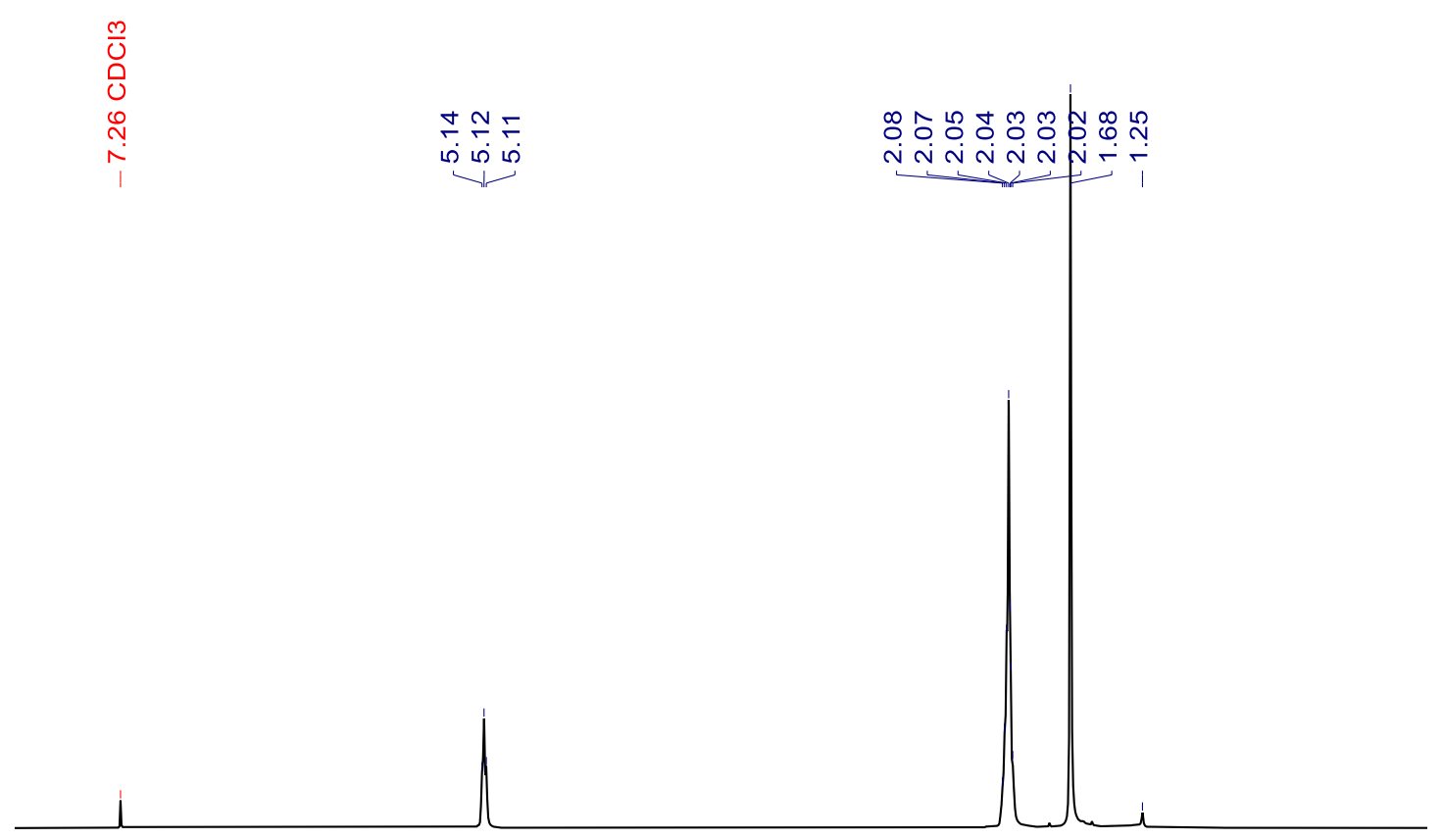

\section{$\begin{array}{lllllllllllllllll}7.5 & 7.0 & 6.5 & 6.0 & 5.5 & 5.0 & 4.5 & 4.0 & 3.5 & 3.0 & 2.5 & 2.0 & 1.5 & 1.0 & 0.5 & 0.0\end{array}$ Chemical shift (ppm)}

Figure S69. ${ }^{1} \mathrm{H}-\mathrm{NMR}$ spectrum of NR

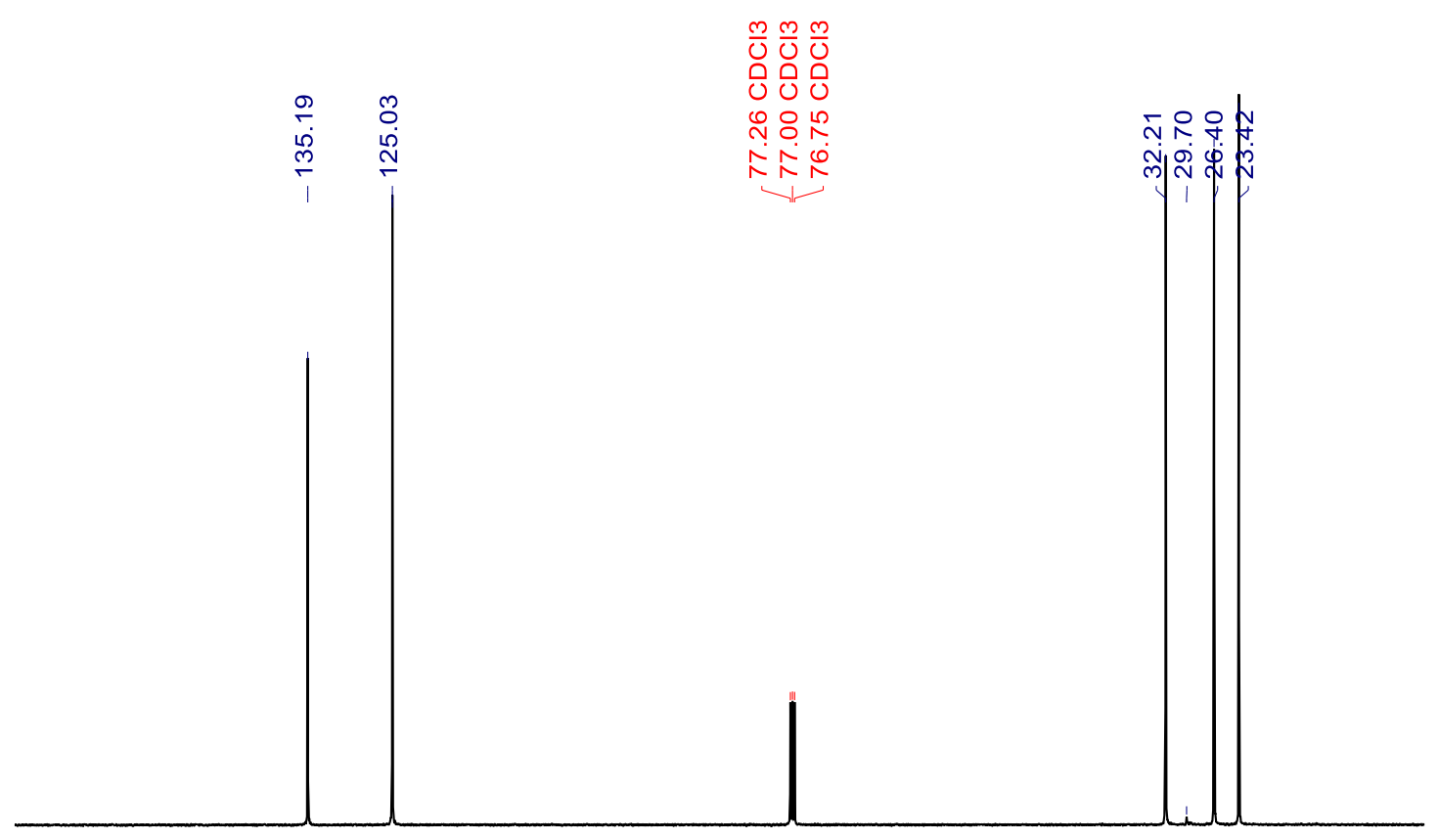

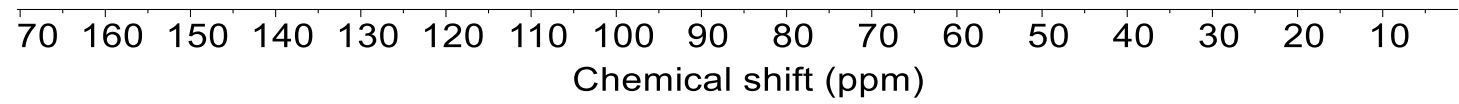

Figure S70. ${ }^{13} \mathrm{C}-\mathrm{NMR}$ spectrum of NR

$38 / 65$ 


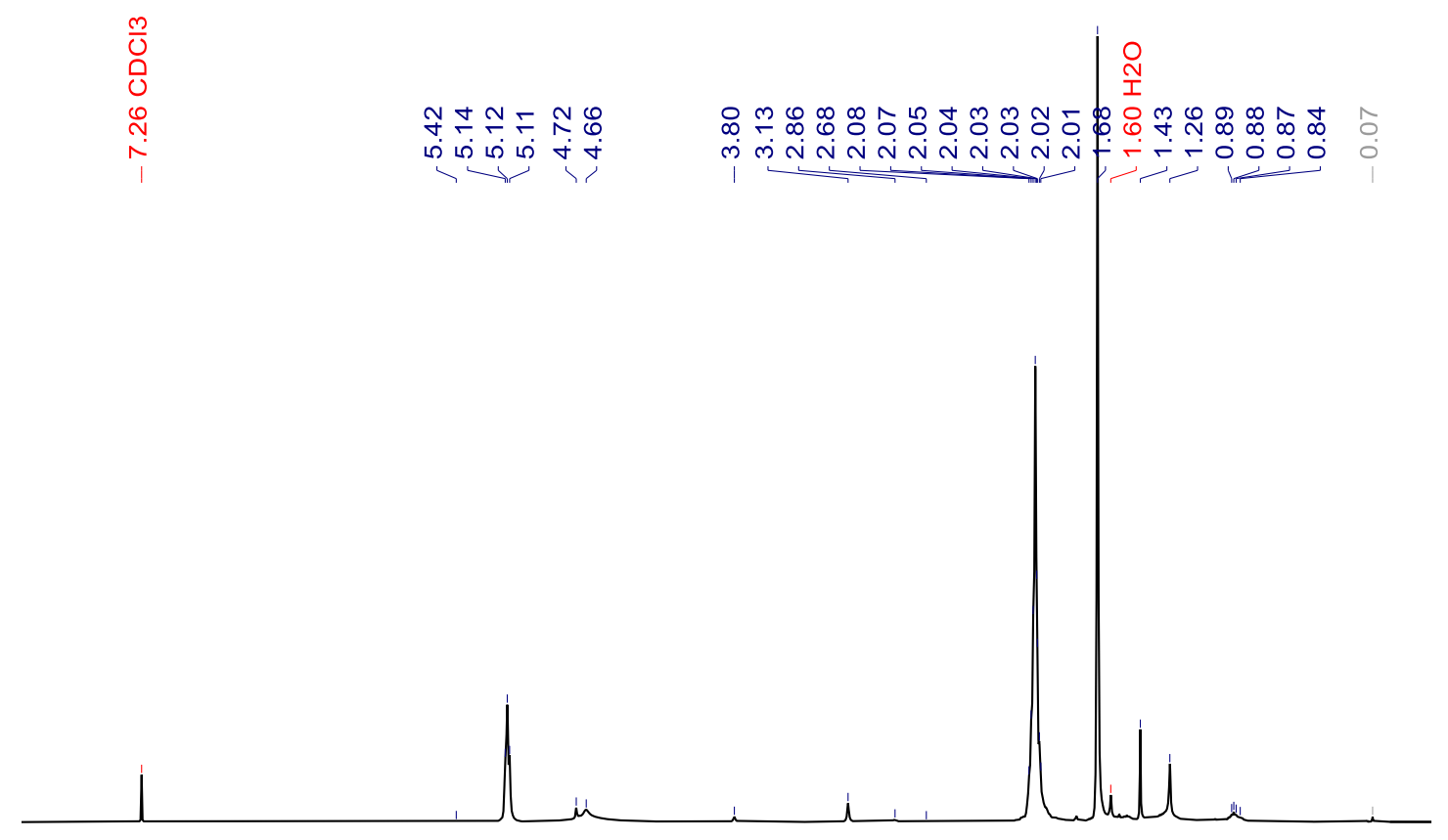

$\begin{array}{llllllllllllllllll}7.5 & 7.0 & 6.5 & 6.0 & 5.5 & 5.0 & \begin{array}{c}4.5 \\ \text { Chemical shift (ppm) }\end{array} & 4.0 & 3.5 & 3.0 & 2.5 & 2.0 & 1.5 & 1.0 & 0.5 & 0.0\end{array}$

Figure S71. ${ }^{1} \mathrm{H}-\mathrm{NMR}$ spectrum of NR after degradation

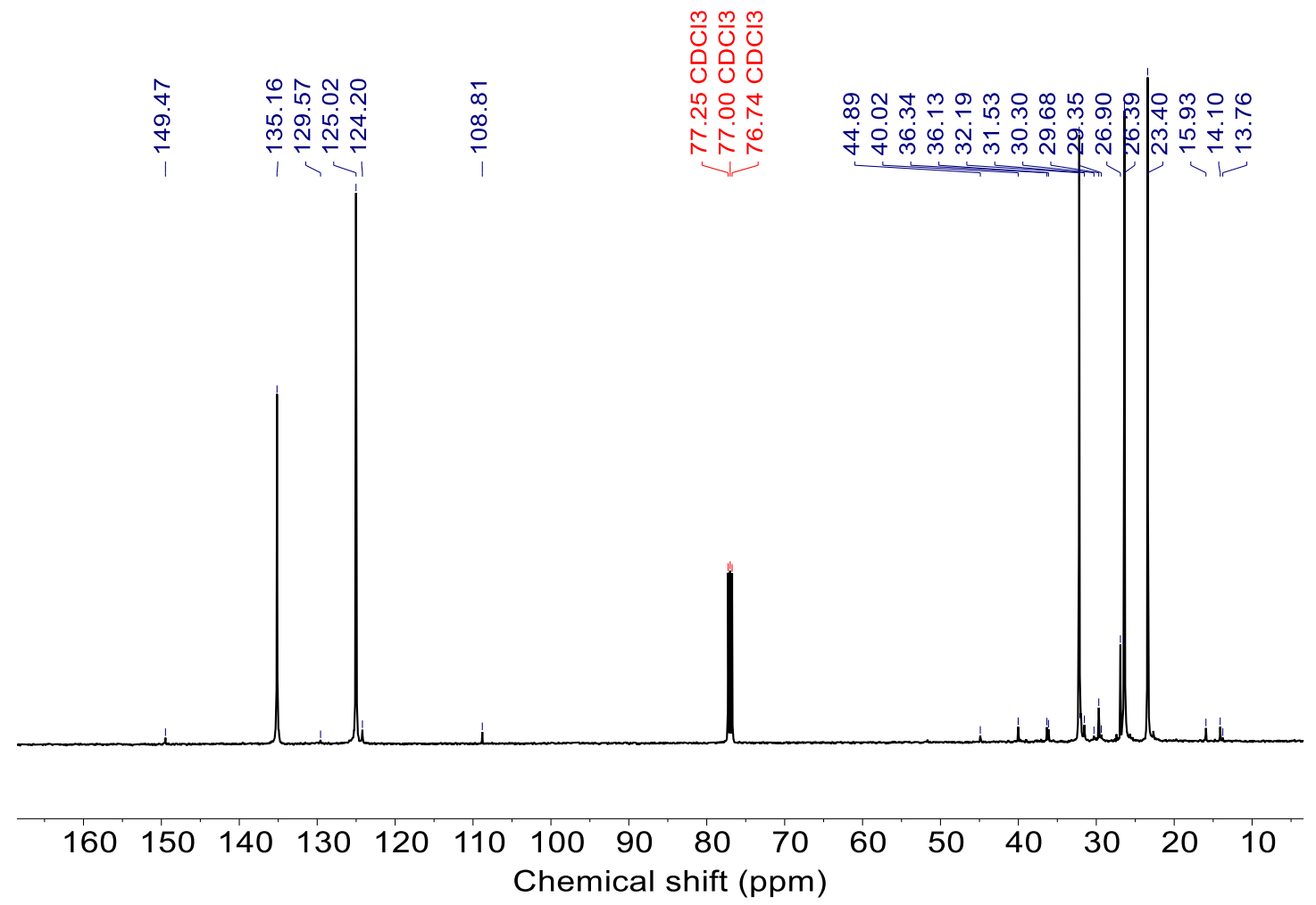

Figure S72. ${ }^{13} \mathrm{C}-\mathrm{NMR}$ spectrum of NR after degradation 


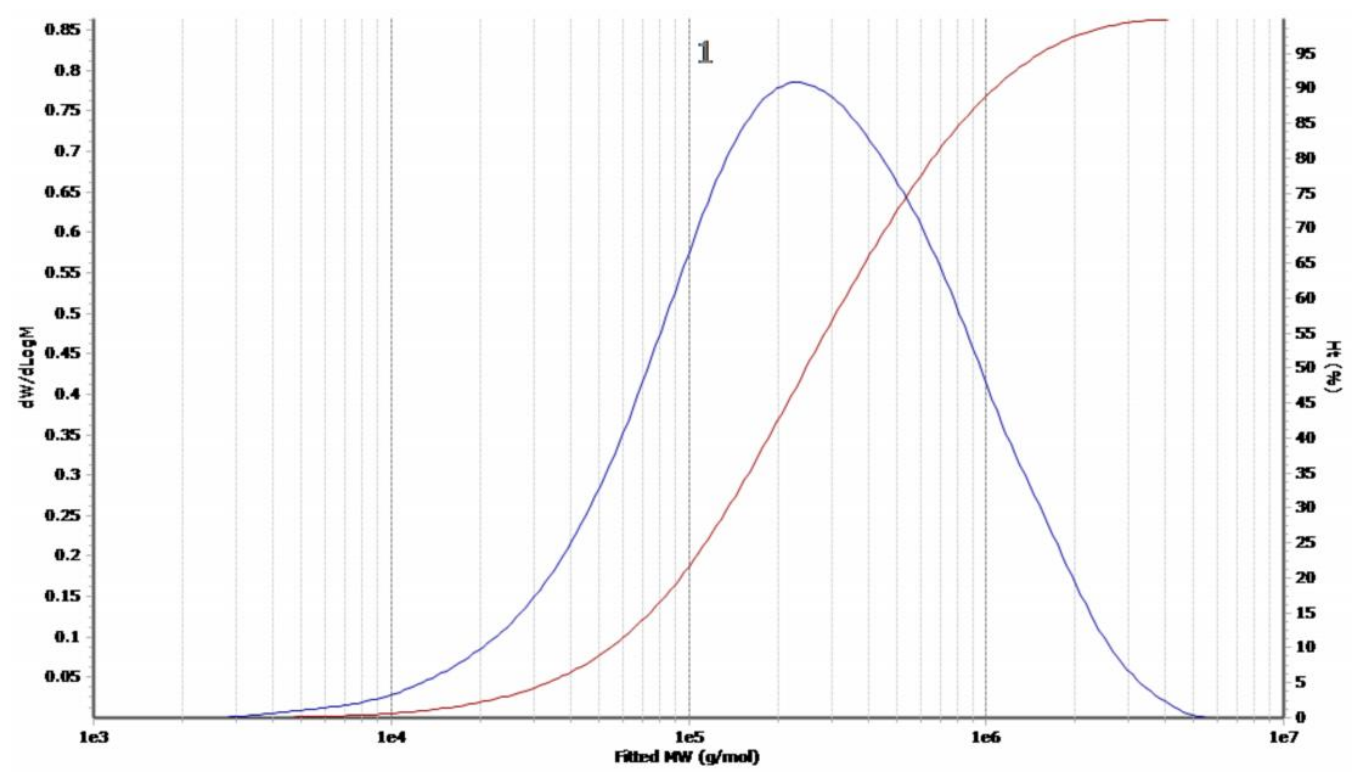

Molecular Weight Averages

\begin{tabular}{|c|c|c|c|c|c|r|r|}
\hline Peak & Mp & Mn & Mw & Mz & Mz+1 & Mv & PD \\
\hline Peak 1 & 233772 & 115696 & 438819 & 1084159 & 1836562 & 981390 & 3.793 \\
\hline
\end{tabular}

Figure S73. GPC data and distribution plot of cis-PB (Table 2. Entry 1)

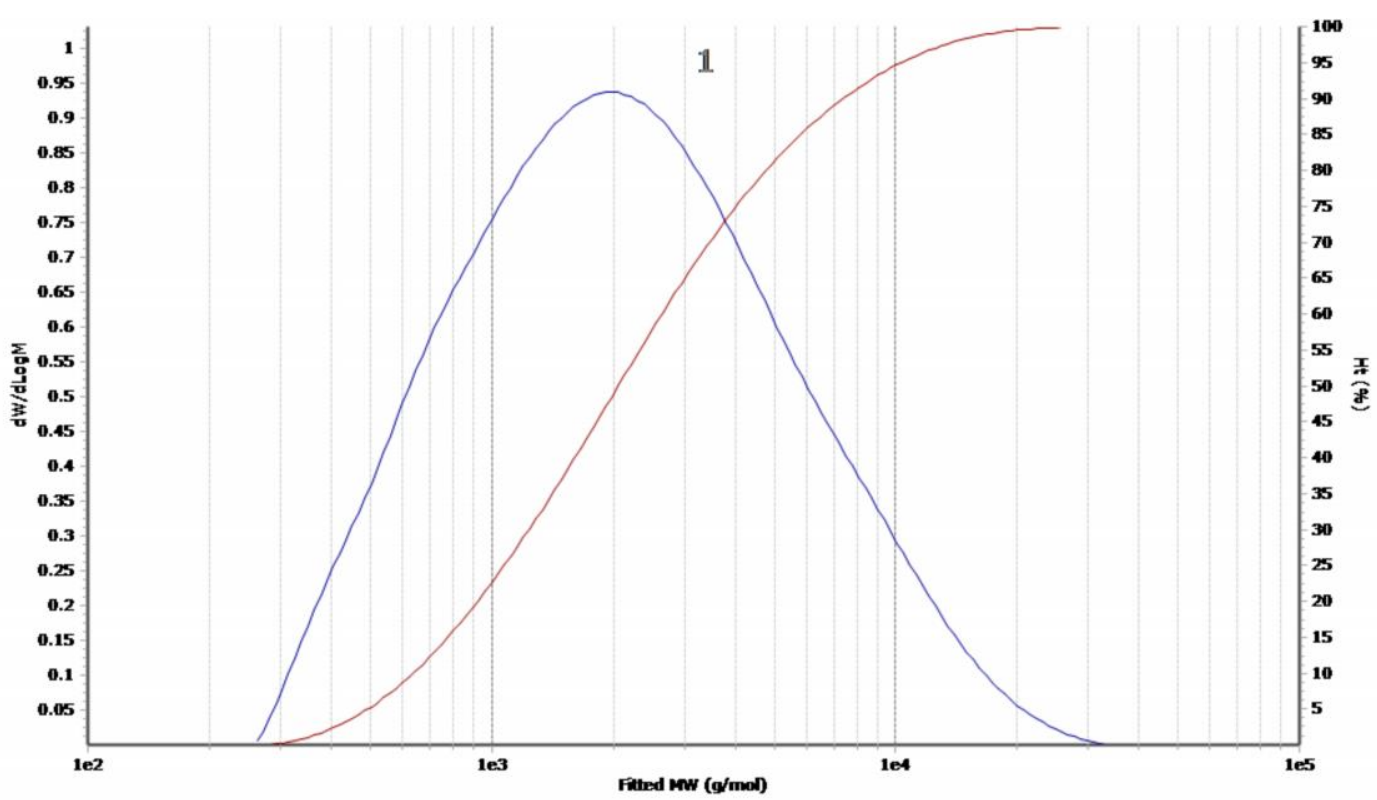

Molecular Weight Averages

\begin{tabular}{|c|c|r|r|r|r|r|r|}
\hline Peak & Mp & \multicolumn{1}{c|}{ Mn } & Mw & \multicolumn{1}{c|}{ Mz } & Mz+1 & \multicolumn{1}{c|}{ Mv } & PD \\
\hline Peak 1 & 1985 & 1443 & 3244 & 6883 & 11334 & 6279 & 2.248 \\
\hline
\end{tabular}

Figure S74. GPC data and distribution plot of degraded cis-PB (Table 2. Entry 2) 


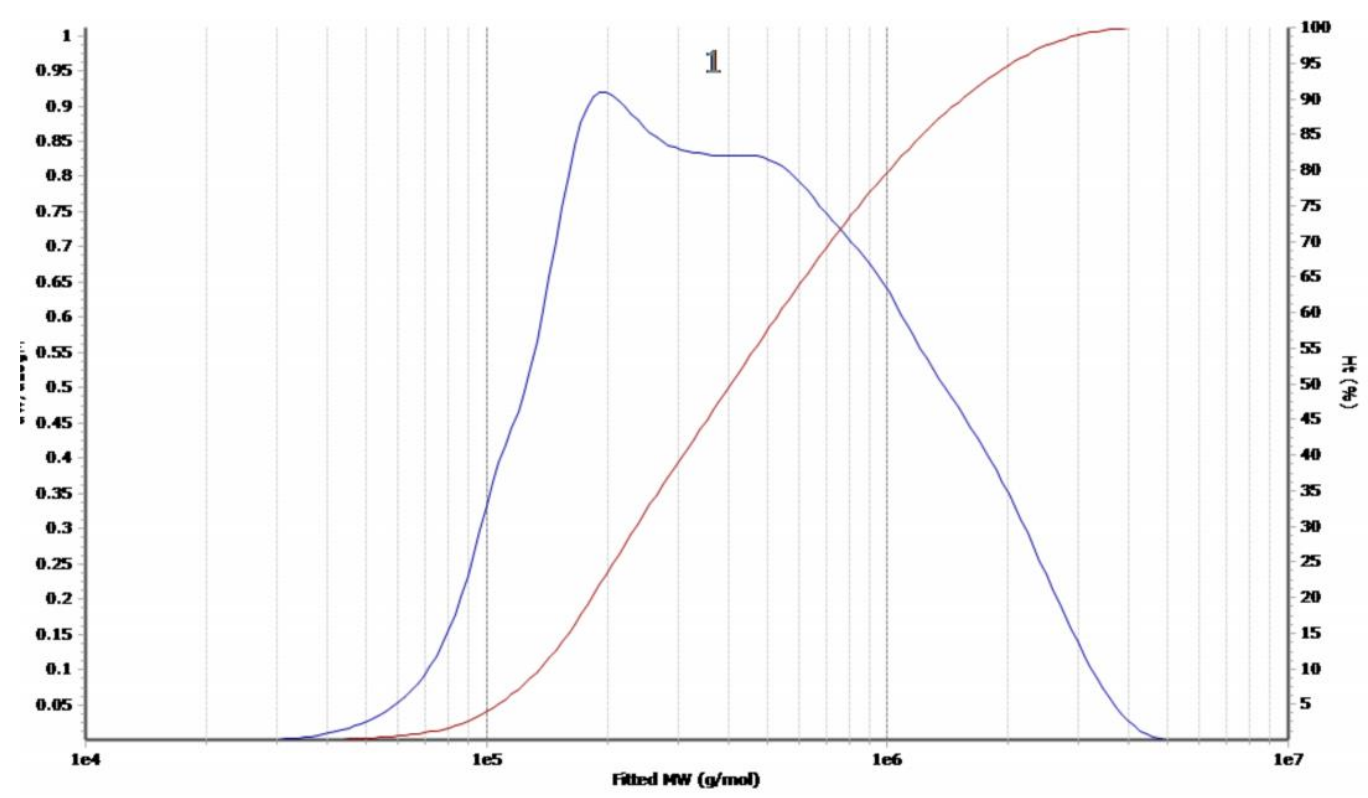

Molecular Weight Averages

\begin{tabular}{|c|c|c|c|c|c|c|r|}
\hline Peak & Mp & Mn & Mw & Mz & Mz+1 & Mv & PD \\
\hline Peak 1 & 195139 & 292271 & 648268 & 1285657 & 1917571 & 1190672 & 2.218 \\
\hline
\end{tabular}

Figure S75. GPC data and distribution plot of HVPB (Table 2. Entry 3)

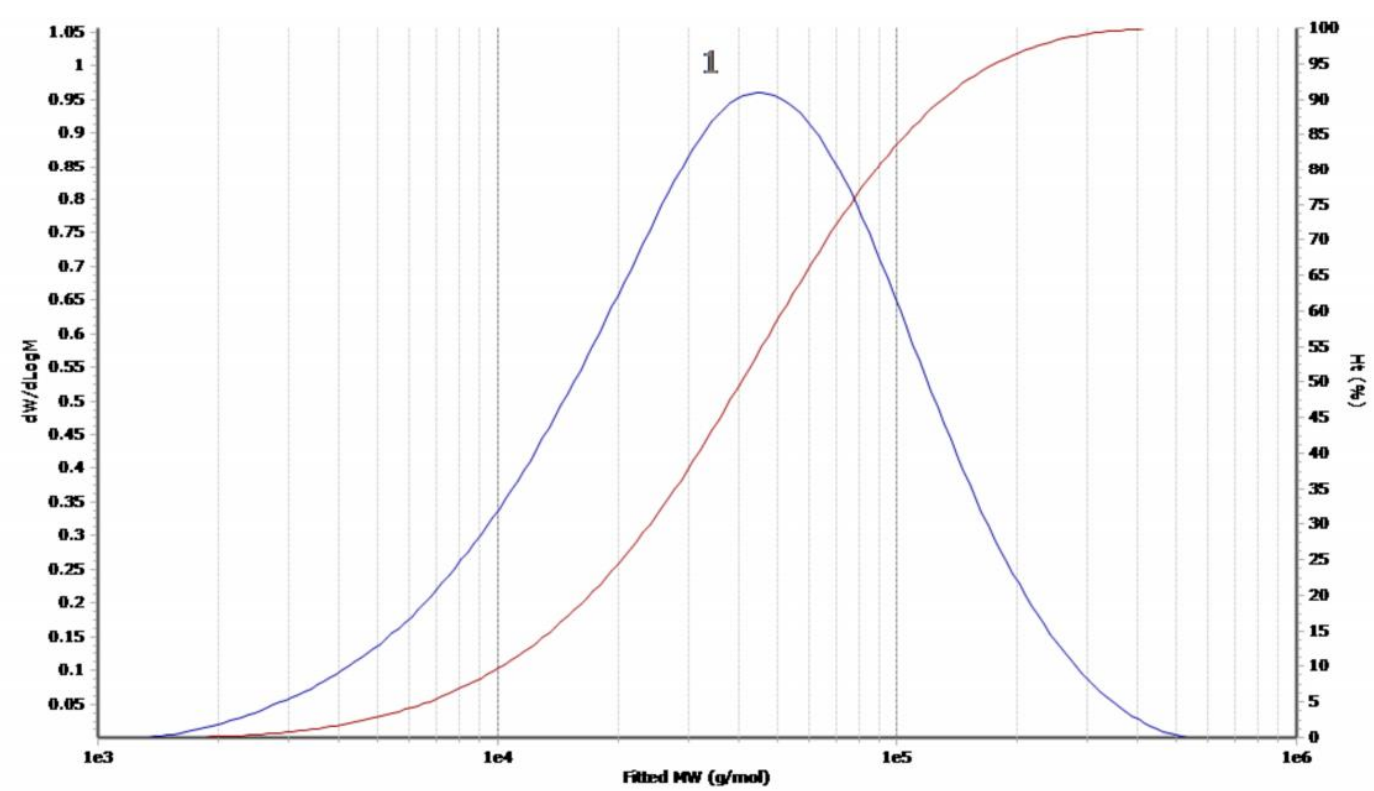

Molecular Weight Averages

\begin{tabular}{|c|c|c|c|c|c|c|r|}
\hline Peak & Mp & Mn & Mw & Mz & Mz+1 & Mv & PD \\
\hline Peak 1 & 45176 & 22778 & 58870 & 116240 & 182952 & 107181 & 2.585 \\
\hline
\end{tabular}

Figure S76. GPC data and distribution plot of degraded HVPB (Table 2. Entry 4) 


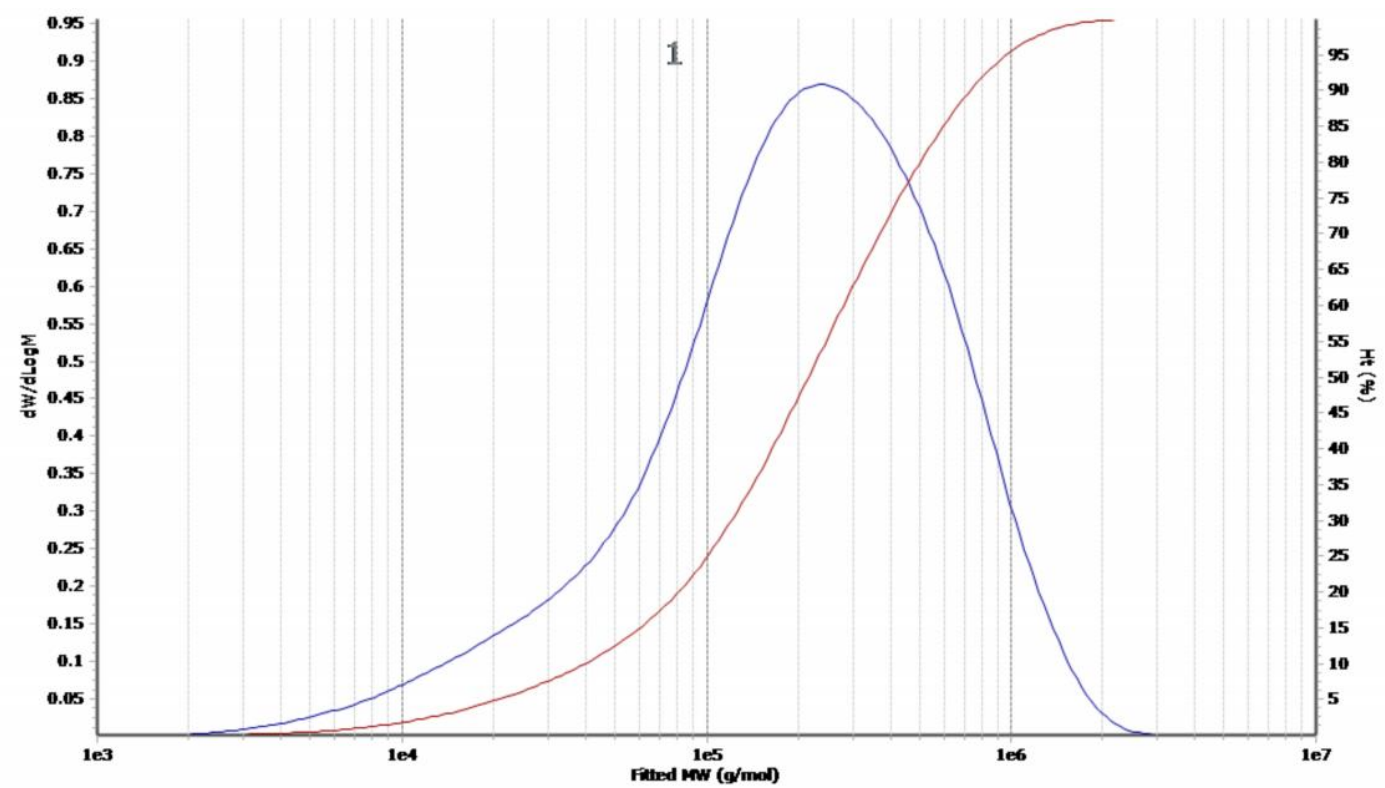

Molecular Weight Averages

Molecular Weight Averages
\begin{tabular}{|c|c|c|c|r|r|r|r|}
\hline Peak & Mp & Mn & Mw & Mz & Mz+1 & Mv & PD \\
\hline Peak 1 & 238033 & 82298 & 317410 & 633739 & 959205 & 588059 & 3.857 \\
\hline
\end{tabular}

Figure S77. GPC data and distribution plot of PI (Table 2. Entry 5)

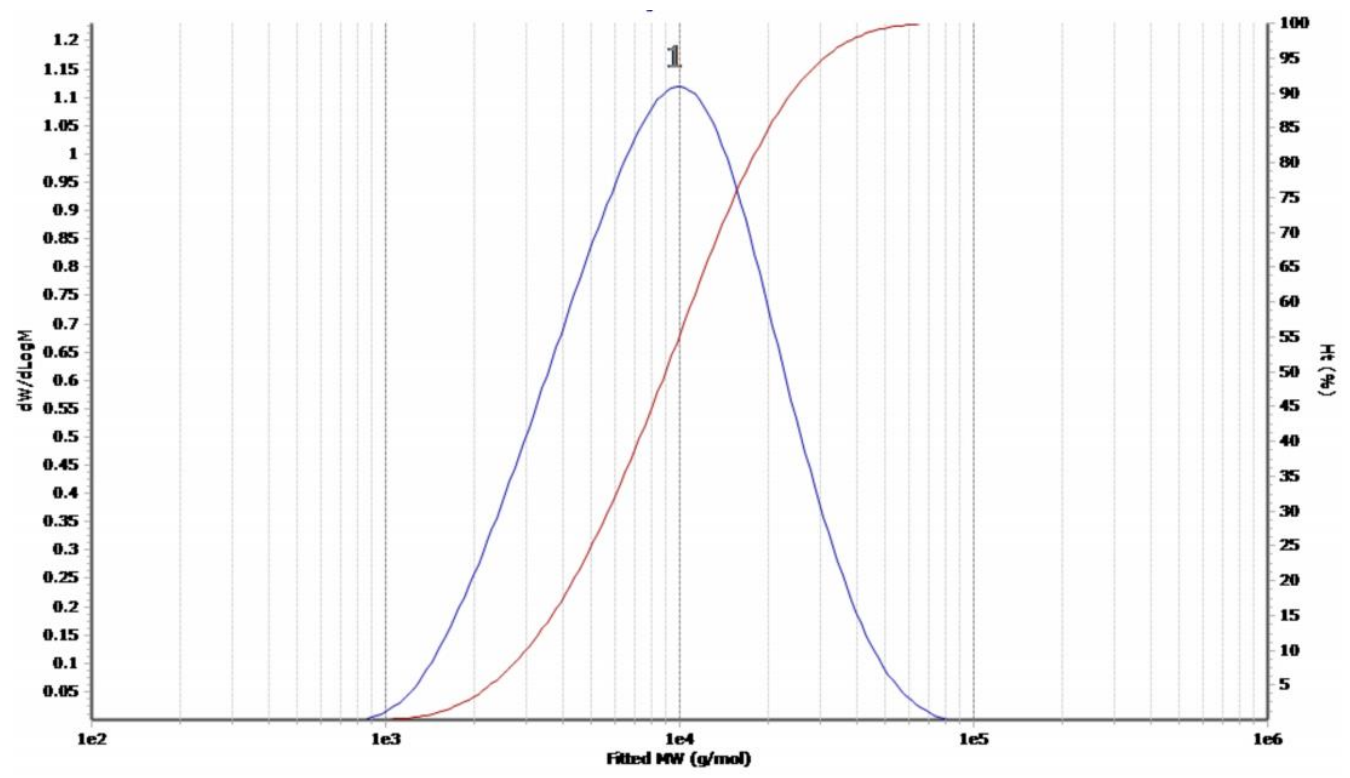

Molecular Weight Averages

\begin{tabular}{|c|c|c|c|r|r|r|r|}
\hline Peak & Mp & Mn & Mw & Mz & \multicolumn{1}{|c|}{ Mz+1 } & Mv & PD \\
\hline Peak 1 & 9907 & 6381 & 11710 & 19485 & 28294 & 18267 & 1.835 \\
\hline
\end{tabular}

Figure S78. GPC data and distribution plot of degraded PI (Table 2. Entry 6) 


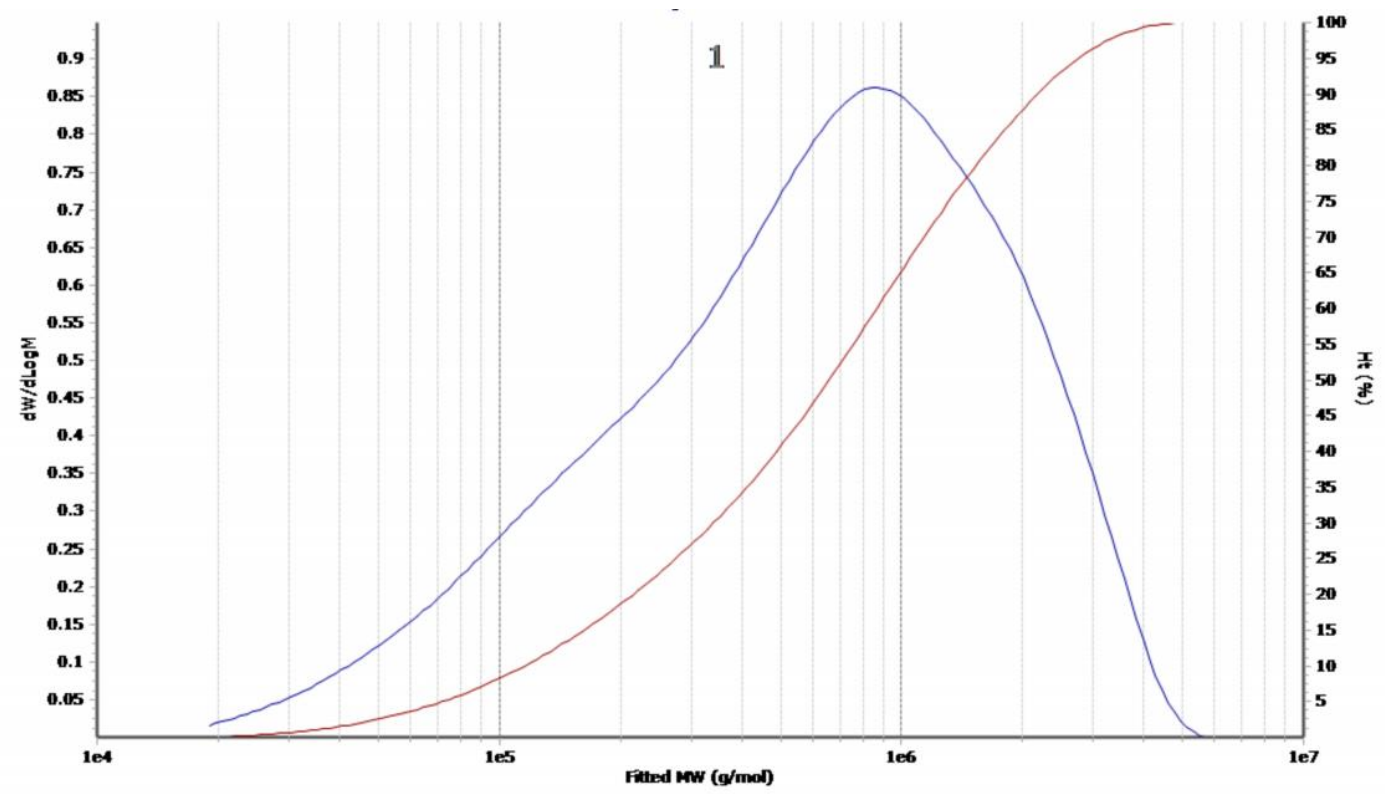

Molecular Weight Averages

\begin{tabular}{|c|c|c|c|c|r|r|r|}
\hline Peak & Mp & Mn & Mw & Mz & Mz+1 & Mv & PD \\
\hline Peak 1 & 858263 & 284867 & 930723 & 1735682 & 2384441 & 1633793 & 3.267 \\
\hline
\end{tabular}

Figure S79. GPC data and distribution plot of NR (Table 2. Entry 7)

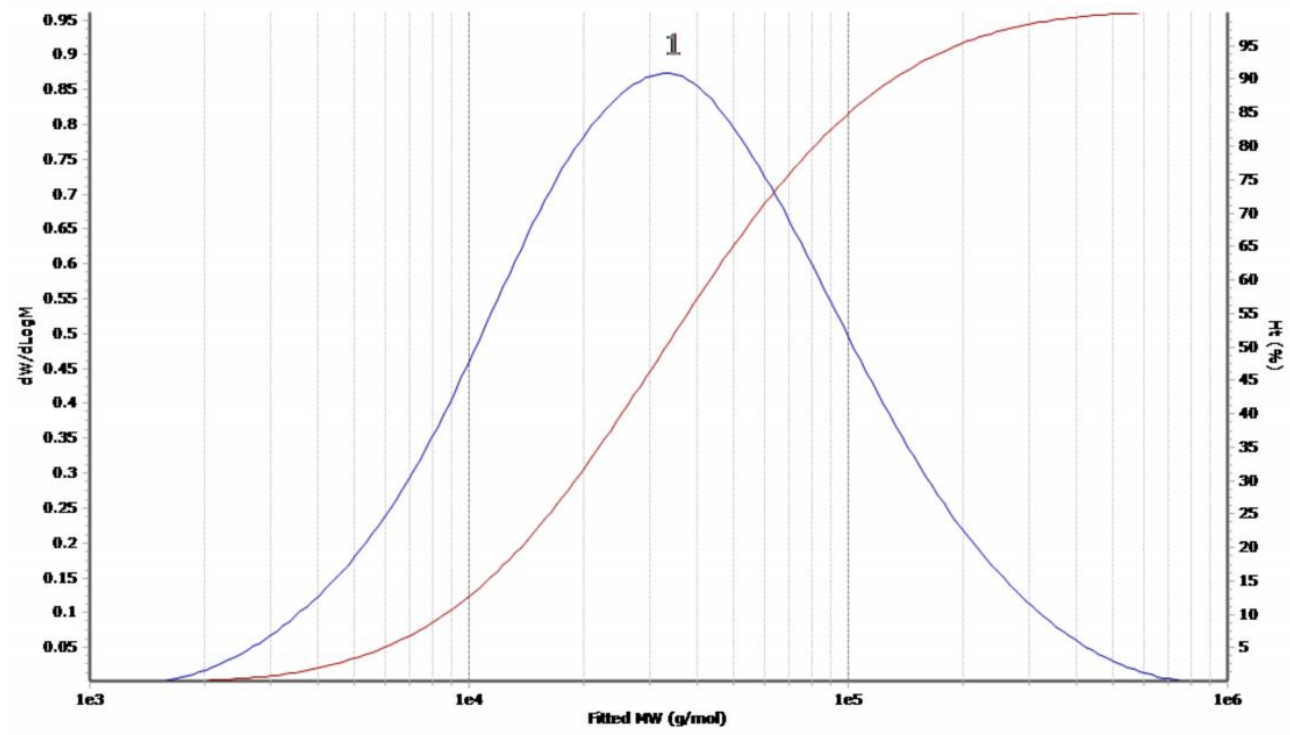

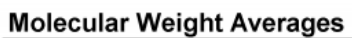

\begin{tabular}{|c|c|c|c|c|r|r|r|}
\hline Peak & Mp & Mn & Mw & Mz & \multicolumn{1}{|c|}{ Mz+1 } & Mv & \multicolumn{1}{c|}{ PD } \\
\hline Peak 1 & 33231 & 19756 & 56821 & 143671 & 262754 & 128376 & 2.876 \\
\hline
\end{tabular}

Figure S80. GPC data and distribution plot of degraded NR (Table 2. Entry 7) 


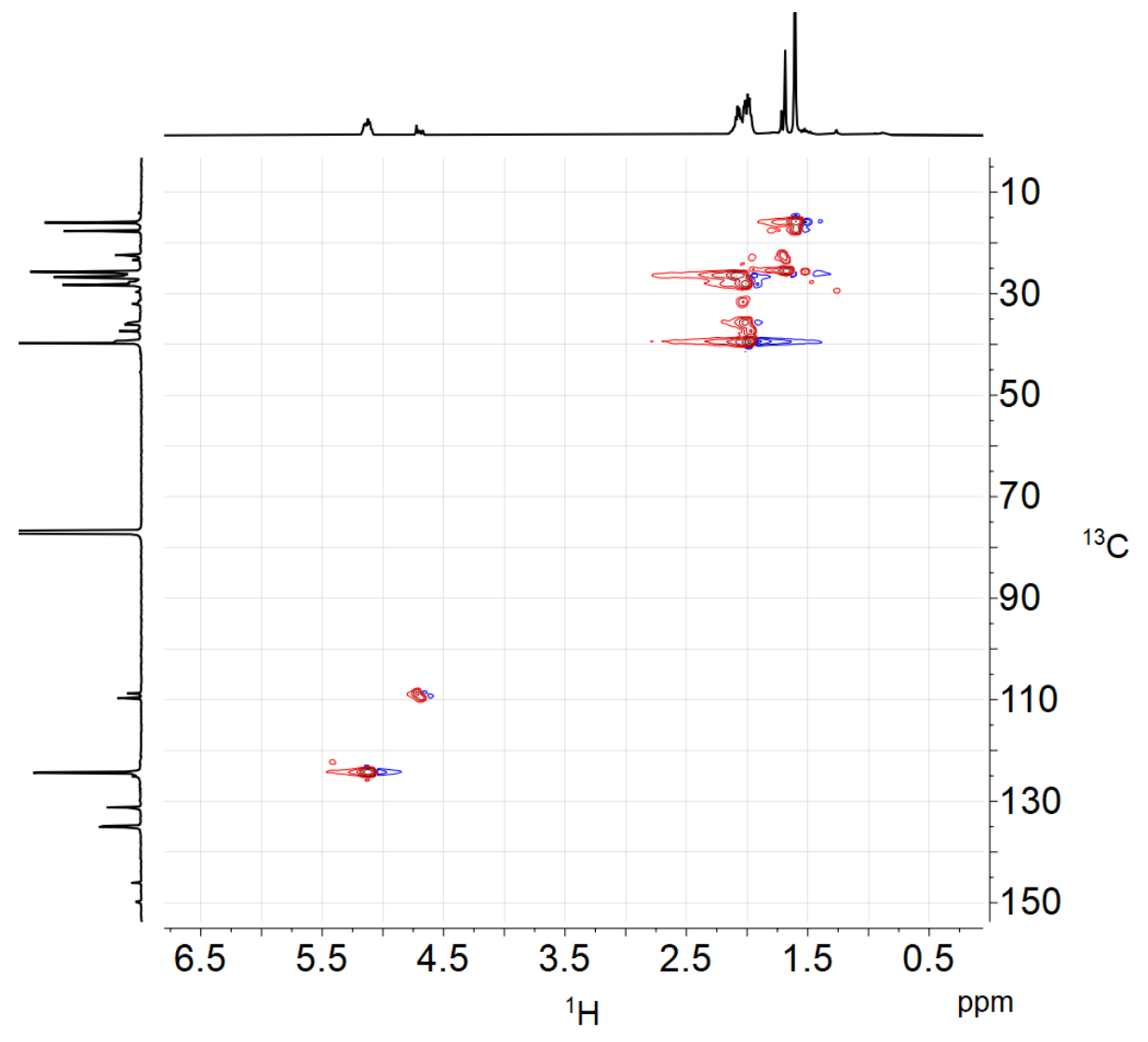

Figure S81. 2D HSQC-NMR spectrum of squalene degradation products 


\section{Cartesian coordinates of all optimized structure}

\section{Ground state 2}

\begin{tabular}{|c|c|c|c|}
\hline Symbol & $\mathbf{X}$ & $\mathbf{Y}$ & $\mathbf{Z}$ \\
\hline $\mathrm{C}$ & -10.05 & 1.810066 & -0.32743 \\
\hline $\mathrm{C}$ & -9.25153 & 0.74807 & -0.11502 \\
\hline $\mathrm{C}$ & -9.4778 & -0.43371 & 0.788867 \\
\hline $\mathrm{C}$ & -9.06022 & -1.77988 & 0.140091 \\
\hline $\mathrm{C}$ & -7.58119 & -1.8785 & -0.24621 \\
\hline $\mathrm{C}$ & -6.66365 & -1.92813 & 0.810318 \\
\hline $\mathrm{C}$ & -5.18708 & -2.0345 & 0.597974 \\
\hline $\mathrm{C}$ & -4.47448 & -0.67933 & 0.232484 \\
\hline $\mathrm{C}$ & -3.01218 & -0.80918 & -0.12167 \\
\hline $\mathrm{C}$ & -2.04778 & -0.45615 & 0.755674 \\
\hline $\mathrm{C}$ & -0.55122 & -0.5547 & 0.6065 \\
\hline $\mathrm{C}$ & 0.14705 & 0.831893 & 0.595031 \\
\hline $\mathrm{C}$ & 1.641464 & 0.73348 & 0.435104 \\
\hline $\mathrm{C}$ & 2.609336 & 1.071939 & 1.305628 \\
\hline $\mathrm{C}$ & 4.090263 & 0.899596 & 1.007938 \\
\hline $\mathrm{C}$ & -9.6452 & 2.905704 & -1.28905 \\
\hline $\mathrm{C}$ & -11.3867 & 2.037818 & 0.341316 \\
\hline $\mathrm{C}$ & -7.29929 & -1.89413 & -1.61837 \\
\hline $\mathrm{C}$ & -2.73915 & -1.40717 & -1.48301 \\
\hline $\mathrm{C}$ & 4.481226 & 0.312914 & -0.36056 \\
\hline $\mathrm{C}$ & 5.971783 & 0.130611 & -0.4915 \\
\hline $\mathrm{C}$ & 6.818444 & 0.727728 & -1.34856 \\
\hline $\mathrm{C}$ & 8.310955 & 0.440666 & -1.37103 \\
\hline $\mathrm{C}$ & 8.856444 & -0.60167 & -0.37816 \\
\hline $\mathrm{C}$ & 10.33069 & -0.84442 & -0.57016 \\
\hline $\mathrm{C}$ & 11.34041 & -0.6243 & 0.290145 \\
\hline $\mathrm{C}$ & 12.76844 & -0.93686 & -0.09567 \\
\hline $\mathrm{C}$ & 11.18212 & -0.06959 & 1.686096 \\
\hline $\mathrm{C}$ & 6.401684 & 1.744204 & -2.3883 \\
\hline $\mathrm{C}$ & 2.352181 & 1.664711 & 2.673363 \\
\hline $\mathrm{H}$ & -8.31233 & 0.69837 & -0.66645 \\
\hline $\mathrm{H}$ & -8.88057 & -0.29901 & 1.701465 \\
\hline $\mathrm{H}$ & -10.5283 & -0.49649 & 1.105207 \\
\hline $\mathrm{H}$ & -9.68278 & -1.94604 & -0.7485 \\
\hline $\mathrm{H}$ & -7.02984 & -1.90126 & 1.83601 \\
\hline $\mathrm{H}$ & -4.68774 & -2.42494 & 1.498544 \\
\hline
\end{tabular}




\begin{tabular}{|c|c|c|c|}
\hline $\mathrm{H}$ & -4.97302 & -2.75472 & -0.21358 \\
\hline $\mathrm{H}$ & -5.0275 & -0.24414 & -0.61034 \\
\hline $\mathrm{H}$ & -4.59331 & -0.00067 & 1.085271 \\
\hline $\mathrm{H}$ & -2.37957 & -0.02948 & 1.705241 \\
\hline $\mathrm{H}$ & -0.12948 & -1.1347 & 1.441795 \\
\hline $\mathrm{H}$ & -0.27673 & -1.09681 & -0.30552 \\
\hline $\mathrm{H}$ & -0.26654 & 1.408946 & -0.24394 \\
\hline $\mathrm{H}$ & -0.12951 & 1.377151 & 1.503341 \\
\hline $\mathrm{H}$ & 1.948341 & 0.305606 & -0.5192 \\
\hline $\mathrm{H}$ & 4.529535 & 0.271224 & 1.799482 \\
\hline $\mathrm{H}$ & 4.58424 & 1.877412 & 1.121033 \\
\hline $\mathrm{H}$ & -8.67988 & 2.690453 & -1.75646 \\
\hline $\mathrm{H}$ & -10.39 & 3.031087 & -2.08998 \\
\hline $\mathrm{H}$ & -9.56997 & 3.879457 & -0.78079 \\
\hline $\mathrm{H}$ & -11.6712 & 1.221681 & 1.009332 \\
\hline $\mathrm{H}$ & -11.3764 & 2.965776 & 0.933206 \\
\hline $\mathrm{H}$ & -12.1856 & 2.157696 & -0.40606 \\
\hline $\mathrm{H}$ & -8.10078 & -1.92526 & -2.35142 \\
\hline $\mathrm{H}$ & -6.28416 & -1.94405 & -2.00321 \\
\hline $\mathrm{H}$ & -3.17457 & -2.41206 & -1.55385 \\
\hline $\mathrm{H}$ & -3.2301 & -0.80845 & -2.2613 \\
\hline $\mathrm{H}$ & -1.67559 & -1.47967 & -1.72785 \\
\hline $\mathrm{H}$ & 3.998239 & -0.66748 & -0.47596 \\
\hline $\mathrm{H}$ & 4.083805 & 0.946938 & -1.158 \\
\hline $\mathrm{H}$ & 6.38875 & -0.58252 & 0.218984 \\
\hline $\mathrm{H}$ & 8.583087 & 0.130023 & -2.39232 \\
\hline $\mathrm{H}$ & 8.849556 & 1.387864 & -1.21447 \\
\hline $\mathrm{H}$ & 8.637214 & -0.28502 & 0.645847 \\
\hline $\mathrm{H}$ & 8.321053 & -1.5494 & -0.53111 \\
\hline $\mathrm{H}$ & 10.59568 & -1.24847 & -1.54962 \\
\hline $\mathrm{H}$ & 12.83777 & -1.33882 & -1.11084 \\
\hline $\mathrm{H}$ & 13.39792 & -0.03796 & -0.04083 \\
\hline $\mathrm{H}$ & 13.2114 & -1.67025 & 0.592227 \\
\hline $\mathrm{H}$ & 10.1487 & 0.171729 & 1.941148 \\
\hline $\mathrm{H}$ & 11.55412 & -0.78635 & 2.430878 \\
\hline $\mathrm{H}$ & 11.78078 & 0.843295 & 1.807392 \\
\hline $\mathrm{H}$ & 5.33969 & 1.991212 & -2.34897 \\
\hline $\mathrm{H}$ & 6.967423 & 2.677268 & -2.2633 \\
\hline $\mathrm{H}$ & 6.623699 & 1.378614 & -3.40019 \\
\hline $\mathrm{H}$ & 1.290422 & 1.774157 & 2.89913 \\
\hline $\mathrm{H}$ & 2.798268 & 1.037396 & 3.457385 \\
\hline
\end{tabular}




$$
\begin{array}{rrrr}
\mathrm{H} & 2.819416 & 2.655385 & 2.763162 \\
\mathrm{H} & -9.30374 & -2.5779 & 0.85702
\end{array}
$$

\begin{tabular}{|c|c|c|c|}
\hline Symbol & $\mathbf{X}$ & $\mathbf{Y}$ & $\mathbf{Z}$ \\
\hline $\mathrm{C}$ & -10.9695 & -0.54418 & 0.553332 \\
\hline $\mathrm{C}$ & -9.79847 & -0.00575 & 0.168389 \\
\hline $\mathrm{C}$ & -9.50729 & 0.857955 & -1.02809 \\
\hline $\mathrm{C}$ & -8.78748 & 2.186751 & -0.66701 \\
\hline $\mathrm{C}$ & -7.39327 & 2.016854 & -0.07535 \\
\hline $\mathrm{C}$ & -6.37047 & 1.560128 & -0.99913 \\
\hline $\mathrm{C}$ & -5.03914 & 1.368236 & -0.72162 \\
\hline $\mathrm{C}$ & -4.25266 & -0.9208 & -0.00362 \\
\hline $\mathrm{C}$ & -2.87179 & -0.69213 & 0.177023 \\
\hline $\mathrm{C}$ & -1.92623 & -0.71026 & -0.8417 \\
\hline $\mathrm{C}$ & -0.45532 & -0.44893 & -0.69294 \\
\hline $\mathrm{C}$ & 0.43103 & -1.73636 & -0.54456 \\
\hline $\mathrm{C}$ & 1.89436 & -1.43223 & -0.37291 \\
\hline $\mathrm{C}$ & 2.905621 & -1.60984 & -1.24411 \\
\hline $\mathrm{C}$ & 4.341619 & -1.20495 & -0.95185 \\
\hline $\mathrm{C}$ & -11.0573 & -1.39447 & 1.801279 \\
\hline $\mathrm{C}$ & -12.2825 & -0.38517 & -0.17849 \\
\hline $\mathrm{C}$ & -7.20647 & 2.290224 & 1.247576 \\
\hline $\mathrm{C}$ & -2.43948 & -0.28612 & 1.584258 \\
\hline $\mathrm{C}$ & 4.635063 & -0.54886 & 0.409943 \\
\hline $\mathrm{C}$ & 6.077672 & -0.13093 & 0.535201 \\
\hline $\mathrm{C}$ & 7.009531 & -0.57259 & 1.398222 \\
\hline $\mathrm{C}$ & 8.439661 & -0.05726 & 1.407665 \\
\hline $\mathrm{C}$ & 8.814313 & 1.03618 & 0.390839 \\
\hline $\mathrm{C}$ & 10.2356 & 1.50385 & 0.563752 \\
\hline $\mathrm{C}$ & 11.25986 & 1.427183 & -0.30415 \\
\hline $\mathrm{C}$ & 12.62653 & 1.960947 & 0.061235 \\
\hline $\mathrm{C}$ & 11.17654 & 0.831677 & -1.68976 \\
\hline $\mathrm{C}$ & 6.758685 & -1.62335 & 2.457013 \\
\hline $\mathrm{C}$ & 2.742008 & -2.23846 & -2.61038 \\
\hline $\mathrm{H}$ & -8.9278 & -0.20047 & 0.79391 \\
\hline $\mathrm{H}$ & -8.86242 & 0.29645 & -1.71863 \\
\hline $\mathrm{H}$ & -10.4244 & 1.093855 & -1.58084 \\
\hline $\mathrm{H}$ & -9.42005 & 2.74866 & 0.031201 \\
\hline $\mathrm{H}$ & -6.7191 & 1.294126 & -1.9982 \\
\hline $\mathrm{H}$ & -4.32806 & 1.133112 & -1.50474 \\
\hline
\end{tabular}

\section{Transition state TS1}




\begin{tabular}{|c|c|c|c|}
\hline $\mathrm{H}$ & -4.60146 & 1.727876 & 0.203556 \\
\hline $\mathrm{H}$ & -4.91421 & -1.01381 & 0.851652 \\
\hline $\mathrm{H}$ & -4.62462 & -1.31684 & -0.94462 \\
\hline $\mathrm{H}$ & -2.25868 & -1.02082 & -1.83398 \\
\hline $\mathrm{H}$ & -0.06624 & 0.099033 & -1.56643 \\
\hline $\mathrm{H}$ & -0.25143 & 0.19473 & 0.174852 \\
\hline $\mathrm{H}$ & 0.068985 & -2.28596 & 0.334513 \\
\hline $\mathrm{H}$ & 0.243642 & -2.38377 & -1.40718 \\
\hline $\mathrm{H}$ & 2.131865 & -0.95546 & 0.578592 \\
\hline $\mathrm{H}$ & 4.678905 & -0.5227 & -1.74957 \\
\hline $\mathrm{H}$ & 4.98517 & -2.09355 & -1.05437 \\
\hline $\mathrm{H}$ & -10.0867 & -1.48261 & 2.297651 \\
\hline $\mathrm{H}$ & -11.772 & -0.97064 & 2.522105 \\
\hline $\mathrm{H}$ & -11.4144 & -2.40847 & 1.568378 \\
\hline $\mathrm{H}$ & -12.2074 & 0.255466 & -1.05964 \\
\hline $\mathrm{H}$ & -12.6663 & -1.36223 & -0.50655 \\
\hline $\mathrm{H}$ & -13.0486 & 0.041572 & 0.484845 \\
\hline $\mathrm{H}$ & -8.02392 & 2.651937 & 1.865604 \\
\hline $\mathrm{H}$ & -6.24459 & 2.157502 & 1.733442 \\
\hline $\mathrm{H}$ & -2.05686 & 0.744176 & 1.6104 \\
\hline $\mathrm{H}$ & -3.28233 & -0.34186 & 2.280208 \\
\hline $\mathrm{H}$ & -1.64109 & -0.93231 & 1.973072 \\
\hline $\mathrm{H}$ & 4.001671 & 0.342939 & 0.51461 \\
\hline $\mathrm{H}$ & 4.342746 & -1.22873 & 1.215056 \\
\hline $\mathrm{H}$ & 6.376774 & 0.626356 & -0.18904 \\
\hline $\mathrm{H}$ & 8.66391 & 0.312652 & 2.421004 \\
\hline $\mathrm{H}$ & 9.118471 & -0.91243 & 1.265897 \\
\hline $\mathrm{H}$ & 8.638932 & 0.669587 & -0.62484 \\
\hline $\mathrm{H}$ & 8.141907 & 1.894388 & 0.530798 \\
\hline $\mathrm{H}$ & 10.44444 & 1.958913 & 1.534464 \\
\hline $\mathrm{H}$ & 12.64223 & 2.38422 & 1.070043 \\
\hline $\mathrm{H}$ & 13.38627 & 1.168599 & 0.011693 \\
\hline $\mathrm{H}$ & 12.94563 & 2.743205 & -0.64144 \\
\hline $\mathrm{H}$ & 10.19061 & 0.429191 & -1.92864 \\
\hline $\mathrm{H}$ & 11.42592 & 1.584738 & -2.44981 \\
\hline $\mathrm{H}$ & 11.90811 & 0.020309 & -1.80417 \\
\hline $\mathrm{H}$ & 5.746588 & -2.02959 & 2.427858 \\
\hline $\mathrm{H}$ & 7.459723 & -2.46138 & 2.343146 \\
\hline $\mathrm{H}$ & 6.926166 & -1.21116 & 3.461686 \\
\hline $\mathrm{H}$ & 1.712169 & -2.53209 & -2.81911 \\
\hline $\mathrm{H}$ & 3.05788 & -1.54233 & -3.40031 \\
\hline
\end{tabular}




$\begin{array}{llrr}\mathrm{H} & 3.376092 & -3.1317 & -2.71024 \\ \mathrm{H} & -8.71641 & 2.783165 & -1.58831\end{array}$

\section{Ground state 3}

\begin{tabular}{llrr} 
Symbol & X & \multicolumn{1}{l}{ Y } \\
C & 2.390922 & 0.278363 & -0.13418 \\
C & 1.061793 & 0.167276 & -0.3082 \\
C & 0.245512 & -1.08039 & -0.51454 \\
C & -0.84419 & -1.29229 & 0.569713 \\
C & -1.93309 & -0.23655 & 0.585469 \\
C & -2.78887 & -0.16873 & -0.6199 \\
C & -3.31741 & 0.941793 & -1.15352 \\
C & 3.033789 & 1.633398 & 0.056075 \\
C & 3.365081 & -0.87583 & -0.11541 \\
C & -2.13196 & 0.56346 & 1.647506 \\
H & 0.482305 & 1.090765 & -0.29934 \\
H & -0.24684 & -1.02811 & -1.49654 \\
H & 0.881237 & -1.97053 & -0.5379 \\
H & -0.36875 & -1.34323 & 1.555094 \\
H & -2.97499 & -1.12165 & -1.11839 \\
H & -3.95371 & 0.896591 & -2.03225 \\
H & -3.12261 & 1.925877 & -0.73551 \\
H & 2.296342 & 2.440921 & 0.033444 \\
H & 3.567627 & 1.685229 & 1.014801 \\
H & 3.779166 & 1.829787 & -0.72669 \\
H & 2.887495 & -1.85108 & -0.22707 \\
H & 4.103038 & -0.76982 & -0.92209 \\
H & 3.932816 & -0.88465 & 0.82463 \\
H & -1.48658 & 0.513186 & 2.519868 \\
H & -2.95221 & 1.274431 & 1.680103 \\
H & -1.31376 & -2.2707 & 0.38972
\end{tabular}

Ground state 4

\begin{tabular}{llrl} 
Symbol & X & \multicolumn{1}{l}{ Y } & \multicolumn{1}{l}{} \\
C & 8.619279 & -0.40936 & -0.69212 \\
C & 7.228071 & -0.48478 & -0.81586 \\
C & 6.325785 & 0.592433 & -0.79533 \\
C & 4.836452 & 0.489139 & -0.74991 \\
C & 4.184663 & 0.49294 & 0.702031 \\
C & 2.690478 & 0.349865 & 0.681856 \\
C & 1.745918 & 1.308644 & 0.780502 \\
C & 0.256628 & 1.038213 & 0.644462
\end{tabular}




\begin{tabular}{|c|c|c|c|}
\hline $\mathrm{C}$ & 6.658421 & -1.89128 & -1.00942 \\
\hline $\mathrm{C}$ & -0.18 & -0.41154 & 0.361645 \\
\hline $\mathrm{C}$ & -1.6699 & -0.52481 & 0.167463 \\
\hline $\mathrm{C}$ & -2.56795 & -1.20083 & 0.905783 \\
\hline $\mathrm{C}$ & -4.055 & -1.21899 & 0.590376 \\
\hline $\mathrm{C}$ & -4.53467 & -0.44141 & -0.64865 \\
\hline $\mathrm{C}$ & -6.01332 & -0.60878 & -0.88174 \\
\hline $\mathrm{C}$ & -6.98035 & 0.325612 & -0.86521 \\
\hline $\mathrm{C}$ & -8.42247 & -0.04639 & -1.12635 \\
\hline $\mathrm{C}$ & -6.75693 & 1.79616 & -0.60192 \\
\hline $\mathrm{C}$ & -2.21888 & -2.019 & 2.129258 \\
\hline $\mathrm{C}$ & 2.05342 & 2.761945 & 1.064951 \\
\hline $\mathrm{H}$ & 9.245459 & -1.29101 & -0.79886 \\
\hline $\mathrm{H}$ & 9.124123 & 0.544331 & -0.54665 \\
\hline $\mathrm{H}$ & 6.741374 & 1.591961 & -0.64758 \\
\hline $\mathrm{H}$ & 4.3599 & 1.329561 & -1.28394 \\
\hline $\mathrm{H}$ & 4.47568 & -0.42196 & -1.25141 \\
\hline $\mathrm{H}$ & 4.62948 & -0.34158 & 1.25727 \\
\hline $\mathrm{H}$ & 4.508462 & 1.408826 & 1.206725 \\
\hline $\mathrm{H}$ & 2.348586 & -0.66279 & 0.464521 \\
\hline $\mathrm{H}$ & -0.14634 & 1.686144 & -0.15261 \\
\hline $\mathrm{H}$ & -0.25125 & 1.378891 & 1.56211 \\
\hline $\mathrm{H}$ & 6.0672 & -1.9656 & -1.93308 \\
\hline $\mathrm{H}$ & 7.463415 & -2.63197 & -1.06551 \\
\hline $\mathrm{H}$ & 5.993965 & -2.18365 & -0.1837 \\
\hline $\mathrm{H}$ & 0.320251 & -0.75583 & -0.5541 \\
\hline $\mathrm{H}$ & 0.172223 & -1.06324 & 1.166045 \\
\hline $\mathrm{H}$ & -2.04006 & 0.036158 & -0.69041 \\
\hline $\mathrm{H}$ & -4.37271 & -2.26947 & 0.490531 \\
\hline $\mathrm{H}$ & -4.60235 & -0.84426 & 1.469331 \\
\hline $\mathrm{H}$ & -4.26711 & 0.614394 & -0.54734 \\
\hline $\mathrm{H}$ & -3.99468 & -0.81393 & -1.53062 \\
\hline $\mathrm{H}$ & -6.3239 & -1.63646 & -1.0831 \\
\hline $\mathrm{H}$ & -8.5393 & -1.11805 & -1.31449 \\
\hline $\mathrm{H}$ & -9.06076 & 0.221584 & -0.27282 \\
\hline $\mathrm{H}$ & -8.81933 & 0.49728 & -1.99506 \\
\hline $\mathrm{H}$ & -5.71495 & 2.043992 & -0.39219 \\
\hline $\mathrm{H}$ & -7.07898 & 2.39672 & -1.46377 \\
\hline $\mathrm{H}$ & -7.361 & 2.13004 & 0.252747 \\
\hline $\mathrm{H}$ & -1.15863 & -1.97871 & 2.383065 \\
\hline $\mathrm{H}$ & -2.78635 & -1.67014 & 3.002985 \\
\hline
\end{tabular}




$\begin{array}{rrrr}\mathrm{H} & -2.48956 & -3.07398 & 1.982766 \\ \mathrm{H} & 3.123329 & 2.94978 & 1.168648 \\ \mathrm{H} & 1.677681 & 3.409406 & 0.259435 \\ \mathrm{H} & 1.558235 & 3.096781 & 1.990073\end{array}$

\section{Ground state 6}

$\begin{array}{lrrr}\text { Symbol } & \mathbf{X} & \mathbf{Y} & \mathbf{Z} \\ \mathrm{C} & 10.72012 & 1.692825 & 0.182949 \\ \mathrm{C} & 9.665573 & 0.935397 & -0.18654 \\ \mathrm{C} & 9.566239 & -0.12076 & -1.26517 \\ \mathrm{C} & 9.011247 & -1.41522 & -0.72586 \\ \mathrm{C} & 7.640497 & -1.68107 & -0.63151 \\ \mathrm{C} & 6.593255 & -0.82605 & -1.01886 \\ \mathrm{C} & 5.132214 & -1.14993 & -0.95709 \\ \mathrm{C} & 4.371758 & -0.62851 & 0.327944 \\ \mathrm{C} & 2.894124 & -0.94763 & 0.347798 \\ \mathrm{C} & 1.979595 & -0.0236 & -0.01495 \\ \mathrm{C} & 0.484837 & -0.16602 & -0.14334 \\ \mathrm{C} & -0.29391 & 0.756442 & 0.831961 \\ \mathrm{C} & -1.78834 & 0.626152 & 0.695881 \\ \mathrm{C} & -2.67909 & 1.541529 & 0.274225 \\ \mathrm{C} & -4.17046 & 1.264398 & 0.175452 \\ \mathrm{C} & 10.63857 & 2.621629 & 1.37419 \\ \mathrm{C} & 12.07054 & 1.678964 & -0.49535 \\ \mathrm{C} & 7.275651 & -3.05865 & -0.08071 \\ \mathrm{C} & 2.554265 & -2.37726 & 0.706038 \\ \mathrm{C} & -4.66762 & -0.13494 & 0.580638 \\ \mathrm{C} & -6.15244 & -0.28635 & 0.369721 \\ \mathrm{C} & -7.10909 & -0.54166 & 1.279413 \\ \mathrm{C} & -8.57995 & -0.66873 & 0.917448 \\ \mathrm{C} & -8.9725 & -0.50165 & -0.56168 \\ \mathrm{C} & -10.4456 & -0.72749 & -0.78194 \\ \mathrm{C} & -11.3773 & 0.145262 & -1.20434 \\ \mathrm{C} & -12.8207 & -0.27681 & -1.35939 \\ \mathrm{C} & -11.1115 & 1.588217 & -1.56328 \\ \mathrm{C} & -6.85042 & -0.73647 & 2.75679 \\ \mathrm{C} & -2.31704 & 2.94893 & -0.14596 \\ \mathrm{H} & 8.74831 & 1.037349 & 0.395299 \\ & 8.911995 & 0.254553 & -2.07445 \\ \mathrm{H} & 10.54988 & -0.28535 & -1.72706 \\ \mathrm{H} & 9.724116 & -2.13341 & -0.32597\end{array}$




$\begin{array}{lrrr}\mathrm{H} & 6.838432 & 0.178291 & -1.36141 \\ \mathrm{H} & 4.594656 & -0.70862 & -1.81405 \\ \mathrm{H} & 4.959957 & -2.23434 & -1.02473 \\ \mathrm{H} & 4.867063 & -1.0636 & 1.205768 \\ \mathrm{H} & 4.524327 & 0.45566 & 0.381623 \\ \mathrm{H} & 2.357933 & 0.973531 & -0.25169 \\ \mathrm{H} & 0.174937 & 0.091036 & -1.16778 \\ \mathrm{H} & 0.166417 & -1.20245 & 0.016166 \\ \mathrm{H} & -0.00188 & 0.487212 & 1.85685 \\ \mathrm{H} & 0.037358 & 1.789545 & 0.685031 \\ \mathrm{H} & -2.1663 & -0.36024 & 0.964358 \\ \mathrm{H} & -4.48874 & 1.465249 & -0.86014 \\ \mathrm{H} & -4.70413 & 2.012777 & 0.78193 \\ \mathrm{H} & 9.64053 & 2.60946 & 1.82428 \\ \mathrm{H} & 11.36342 & 2.340353 & 2.156472 \\ \mathrm{H} & 10.87349 & 3.661762 & 1.097588 \\ \mathrm{H} & 12.11922 & 0.963443 & -1.31946 \\ \mathrm{H} & 12.32483 & 2.671274 & -0.89977 \\ \mathrm{H} & 12.86984 & 1.424856 & 0.219942 \\ \mathrm{H} & 6.738537 & -3.6635 & -0.8251 \\ \mathrm{H} & -5.8048 & -0.59504 & 3.034812 \\ \mathrm{H} & -176414 & -3.61255 & 0.204928 \\ \mathrm{H} & 6.624767 & -2.99339 & 0.802707 \\ \mathrm{H} & 3.025411 & -3.07204 & -0.00124 \\ \mathrm{H} & 2.958235 & -2.62524 & 1.696438 \\ \mathrm{H} & 1.480603 & -2.58467 & 0.717047 \\ \mathrm{H} & -4.14834 & -0.88514 & -0.03233 \\ \mathrm{H} & -4.38717 & -0.33767 & 1.61809 \\ \mathrm{H} & -6.46046 & -0.163 & -0.66812 \\ \mathrm{H} & -8.93487 & -1.65114 & 1.267766 \\ \mathrm{H} & -9.14809 & 0.061945 & 1.513252 \\ \mathrm{H} & -8.66725 & 0.488304 & -0.91371 \\ \mathrm{H} & -8.41119 & -1.23123 & -1.1623 \\ \mathrm{H} & -10.785 & -1.74005 & -0.55353 \\ \mathrm{H} & -12.9692 & -1.32794 & -1.09444 \\ \mathrm{H} & -13.4795 & 0.331476 & -0.72432 \\ \mathrm{H} & & -0.13363 & -2.39296 \\ \mathrm{H} & -11.7805 & 1.781583 & -2.61065 \\ \mathrm{H} & 2.256596 & -0.95405 \\ \mathrm{H} & -584672 & -1.42496 \\ \mathrm{H} & & & \\ \mathrm{H} & & & \\ \mathrm{H} & & & \end{array}$




$\begin{array}{lrrr}\mathrm{H} & -7.14705 & -1.74589 & 3.073061 \\ \mathrm{H} & -1.24537 & 3.147774 & -0.0998 \\ \mathrm{H} & -2.6491 & 3.142258 & -1.17515 \\ \mathrm{H} & -2.82464 & 3.688181 & 0.489271\end{array}$

\section{Transition state TS2}

\begin{tabular}{|c|c|c|c|}
\hline Symbol & $\mathbf{X}$ & $\mathbf{Y}$ & $\mathbf{Z}$ \\
\hline $\mathrm{C}$ & 11.37107 & 0.66352 & $-0.5324 c$ \\
\hline $\mathrm{C}$ & 10.08173 & 0.280251 & -0.59845 \\
\hline $\mathrm{C}$ & 9.473895 & -0.92126 & -1.28546 \\
\hline $\mathrm{C}$ & 8.737467 & -1.81378 & -0.31129 \\
\hline $\mathrm{C}$ & 7.382539 & -1.83747 & -0.09844 \\
\hline $\mathrm{C}$ & 6.418537 & -1.01434 & -0.7878 \\
\hline $\mathrm{C}$ & 5.051752 & -1.01054 & -0.56663 \\
\hline $\mathrm{C}$ & 4.189501 & 0.57236 & 0.991775 \\
\hline $\mathrm{C}$ & 2.791737 & 0.334741 & 0.978324 \\
\hline C & 1.913437 & 0.875481 & 0.052197 \\
\hline $\mathrm{C}$ & 0.439791 & 0.600178 & -0.04461 \\
\hline $\mathrm{C}$ & -0.47181 & 1.680855 & 0.632927 \\
\hline $\mathrm{C}$ & -1.93956 & 1.362192 & 0.540766 \\
\hline $\mathrm{C}$ & -2.89117 & 1.956441 & -0.20347 \\
\hline $\mathrm{C}$ & -4.33963 & 1.495156 & -0.2308 \\
\hline $\mathrm{C}$ & 11.77906 & 1.887685 & 0.256777 \\
\hline $\mathrm{C}$ & 12.52244 & -0.05478 & -1.19739 \\
\hline $\mathrm{C}$ & 6.848896 & -2.80793 & 0.940396 \\
\hline $\mathrm{C}$ & 2.281057 & -0.70315 & 1.974894 \\
\hline $\mathrm{C}$ & -4.71732 & 0.278203 & 0.633573 \\
\hline $\mathrm{C}$ & -6.16218 & -0.11273 & 0.457337 \\
\hline $\mathrm{C}$ & -7.15016 & -0.11392 & 1.369553 \\
\hline $\mathrm{C}$ & -8.57418 & -0.53694 & 1.046914 \\
\hline $\mathrm{C}$ & -8.87519 & -1.00513 & -0.38834 \\
\hline $\mathrm{C}$ & -10.3009 & -1.46525 & -0.54416 \\
\hline $\mathrm{C}$ & -11.2674 & -0.96316 & -1.33286 \\
\hline $\mathrm{C}$ & -12.6504 & -1.57421 & -1.34798 \\
\hline $\mathrm{C}$ & -11.0995 & 0.214653 & -2.26348 \\
\hline $\mathrm{C}$ & -6.97479 & 0.297687 & 2.814512 \\
\hline $\mathrm{C}$ & -2.64209 & 3.154527 & -1.09295 \\
\hline $\mathrm{H}$ & 9.352303 & 0.884738 & -0.0576 \\
\hline $\mathrm{H}$ & 8.783749 & -0.57112 & -2.06512 \\
\hline $\mathrm{H}$ & 10.24868 & -1.49762 & -1.80775 \\
\hline $\mathrm{H}$ & 9.368811 & -2.46385 & 0.293793 \\
\hline
\end{tabular}




\begin{tabular}{|c|c|c|c|}
\hline $\mathrm{H}$ & 6.810035 & -0.26252 & -1.47105 \\
\hline $\mathrm{H}$ & 4.388023 & -0.48064 & -1.24167 \\
\hline $\mathrm{H}$ & 4.574482 & -1.80681 & -0.00544 \\
\hline $\mathrm{H}$ & 4.780321 & 0.267202 & 1.850914 \\
\hline $\mathrm{H}$ & 4.598996 & 1.396816 & 0.413136 \\
\hline $\mathrm{H}$ & 2.305227 & 1.624907 & -0.63794 \\
\hline $\mathrm{H}$ & 0.122934 & 0.53738 & -1.0983 \\
\hline $\mathrm{H}$ & 0.18819 & -0.37388 & 0.39821 \\
\hline $\mathrm{H}$ & -0.18032 & 1.74266 & 1.690001 \\
\hline $\mathrm{H}$ & -0.23467 & 2.655554 & 0.194652 \\
\hline $\mathrm{H}$ & -2.23568 & 0.496257 & 1.133594 \\
\hline $\mathrm{H}$ & -4.61606 & 1.282147 & -1.27655 \\
\hline $\mathrm{H}$ & -4.98279 & 2.34088 & 0.061047 \\
\hline $\mathrm{H}$ & 10.91525 & 2.378298 & 0.715574 \\
\hline $\mathrm{H}$ & 12.4868 & 1.627974 & 1.058801 \\
\hline $\mathrm{H}$ & 12.28987 & 2.624661 & -0.3809 \\
\hline $\mathrm{H}$ & 12.2114 & -0.95584 & -1.73016 \\
\hline $\mathrm{H}$ & 13.03189 & 0.601896 & -1.91812 \\
\hline $\mathrm{H}$ & 13.28064 & -0.34664 & -0.45538 \\
\hline $\mathrm{H}$ & 6.150109 & -3.52662 & 0.492382 \\
\hline $\mathrm{H}$ & 7.662387 & -3.37271 & 1.407696 \\
\hline $\mathrm{H}$ & 6.296193 & -2.28103 & 1.72816 \\
\hline $\mathrm{H}$ & 1.948978 & -1.62199 & 1.470196 \\
\hline $\mathrm{H}$ & 3.068915 & -0.9834 & 2.681112 \\
\hline $\mathrm{H}$ & 1.427116 & -0.33291 & 2.557201 \\
\hline $\mathrm{H}$ & -4.08611 & -0.57185 & 0.339403 \\
\hline $\mathrm{H}$ & -4.48298 & 0.486006 & 1.681312 \\
\hline $\mathrm{H}$ & -6.40919 & -0.4251 & -0.55701 \\
\hline $\mathrm{H}$ & -8.8597 & -1.33925 & 1.746003 \\
\hline $\mathrm{H}$ & -9.2488 & 0.298505 & 1.289965 \\
\hline $\mathrm{H}$ & -8.63867 & -0.20346 & -1.09411 \\
\hline $\mathrm{H}$ & -8.2064 & -1.84199 & -0.63465 \\
\hline $\mathrm{H}$ & -10.5683 & -2.3205 & 0.080336 \\
\hline $\mathrm{H}$ & -12.7278 & -2.42398 & -0.66317 \\
\hline $\mathrm{H}$ & -13.4118 & -0.83523 & -1.06253 \\
\hline $\mathrm{H}$ & -12.9167 & -1.92309 & -2.35536 \\
\hline $\mathrm{H}$ & -10.1039 & 0.659969 & -2.22192 \\
\hline $\mathrm{H}$ & -11.2915 & -0.08383 & -3.30318 \\
\hline $\mathrm{H}$ & -11.8297 & 0.999914 & -2.0255 \\
\hline $\mathrm{H}$ & -5.96646 & 0.645923 & 3.043007 \\
\hline $\mathrm{H}$ & -7.67459 & 1.103666 & 3.07401 \\
\hline
\end{tabular}




$\begin{array}{lrrr}\mathrm{H} & -7.20115 & -0.54046 & 3.487973 \\ \mathrm{H} & -1.60331 & 3.487437 & -1.07236 \\ \mathrm{H} & -2.8989 & 2.924772 & -2.13672 \\ \mathrm{H} & -3.27578 & 4.0028 & -0.79548\end{array}$

\section{Ground state 7}

\begin{tabular}{|c|c|c|c|}
\hline Symbol & $\mathbf{X}$ & $\mathbf{Y}$ & $\mathbf{Z}$ \\
\hline $\mathrm{C}$ & 2.390922 & 0.278363 & -0.13418 \\
\hline $\mathrm{C}$ & 1.061793 & 0.167276 & -0.3082 \\
\hline $\mathrm{C}$ & 0.245512 & -1.08039 & -0.51454 \\
\hline $\mathrm{C}$ & -0.84419 & -1.29229 & 0.569713 \\
\hline $\mathrm{C}$ & -1.93309 & -0.23655 & 0.585469 \\
\hline $\mathrm{C}$ & -2.78887 & -0.16873 & -0.6199 \\
\hline $\mathrm{C}$ & -3.31741 & 0.941793 & -1.15352 \\
\hline $\mathrm{C}$ & 3.033789 & 1.633398 & 0.056075 \\
\hline $\mathrm{C}$ & 3.365081 & -0.87583 & -0.11541 \\
\hline $\mathrm{C}$ & -2.13196 & 0.56346 & 1.647506 \\
\hline $\mathrm{H}$ & 0.482305 & 1.090765 & -0.29934 \\
\hline $\mathrm{H}$ & -0.24684 & -1.02811 & -1.49654 \\
\hline $\mathrm{H}$ & 0.881237 & -1.97053 & -0.5379 \\
\hline $\mathrm{H}$ & -0.36875 & -1.34323 & 1.555094 \\
\hline $\mathrm{H}$ & -2.97499 & -1.12165 & -1.11839 \\
\hline $\mathrm{H}$ & -3.95371 & 0.896591 & -2.03225 \\
\hline $\mathrm{H}$ & -3.12261 & 1.925877 & -0.73551 \\
\hline $\mathrm{H}$ & 2.296342 & 2.440921 & 0.033444 \\
\hline $\mathrm{H}$ & 3.567627 & 1.685229 & 1.014801 \\
\hline $\mathrm{H}$ & 3.779166 & 1.829787 & -0.72669 \\
\hline $\mathrm{H}$ & 2.887495 & -1.85108 & -0.22707 \\
\hline $\mathrm{H}$ & 4.103038 & -0.76982 & -0.92209 \\
\hline $\mathrm{H}$ & 3.932816 & -0.88465 & 0.82463 \\
\hline $\mathrm{H}$ & -1.48658 & 0.513186 & 2.519868 \\
\hline $\mathrm{H}$ & -2.95221 & 1.274431 & 1.680103 \\
\hline $\mathrm{H}$ & -1.31376 & -2.2707 & 0.38972 \\
\hline
\end{tabular}

\section{Ground state 9}

\begin{tabular}{llrl} 
Symbol & \multicolumn{1}{l}{ X } & \multicolumn{1}{l}{ Y } & \multicolumn{1}{l}{} \\
C & 5.333489 & -1.96564 & -0.10725 \\
C & 6.582244 & -2.35378 & 0.216443 \\
C & 7.864201 & -1.99732 & -0.49281 \\
C & 4.87055 & -1.03913 & -1.21479 \\
C & 4.165441 & 0.19591 & -0.6952
\end{tabular}




\begin{tabular}{|c|c|c|c|}
\hline $\mathrm{C}$ & 4.847174 & 1.379434 & -0.40166 \\
\hline $\mathrm{C}$ & 4.418237 & 2.562922 & 0.206231 \\
\hline $\mathrm{C}$ & 3.022375 & 2.758935 & 0.705278 \\
\hline $\mathrm{C}$ & 1.949584 & 3.030507 & -0.41862 \\
\hline $\mathrm{C}$ & 0.559092 & 3.209454 & 0.113892 \\
\hline $\mathrm{C}$ & -0.46323 & 2.332588 & 0.12055 \\
\hline $\mathrm{C}$ & -0.47471 & 0.951686 & -0.48698 \\
\hline C & -0.61774 & -0.20364 & 0.532061 \\
\hline $\mathrm{C}$ & -1.8838 & -0.17641 & 1.426459 \\
\hline $\mathrm{C}$ & -3.24114 & -0.14544 & 0.66516 \\
\hline $\mathrm{C}$ & -3.77197 & -1.45341 & 0.032229 \\
\hline $\mathrm{C}$ & -5.24553 & -1.25267 & -0.45631 \\
\hline C & -5.42067 & -0.28017 & -1.59328 \\
\hline $\mathrm{C}$ & -6.11014 & 0.871043 & -1.60035 \\
\hline $\mathrm{C}$ & -6.87279 & 1.503812 & -0.46834 \\
\hline $\mathrm{C}$ & -1.83163 & -1.30614 & 2.427582 \\
\hline C & -1.90828 & -1.16812 & 3.75551 \\
\hline $\mathrm{C}$ & -2.90915 & -1.99679 & -1.08225 \\
\hline $\mathrm{C}$ & -2.55112 & -3.27774 & -1.21876 \\
\hline $\mathrm{H}$ & 4.526564 & -2.3041 & 0.545925 \\
\hline $\mathrm{H}$ & 6.708915 & -2.96846 & 1.110648 \\
\hline $\mathrm{H}$ & 8.545067 & -1.44348 & 0.170128 \\
\hline $\mathrm{H}$ & 8.406802 & -2.897 & -0.81958 \\
\hline $\mathrm{H}$ & 7.689742 & -1.37656 & -1.37543 \\
\hline $\mathrm{H}$ & 5.728381 & -0.74495 & -1.83638 \\
\hline $\mathrm{H}$ & 4.203641 & -1.61778 & -1.88077 \\
\hline $\mathrm{H}$ & 3.115513 & 0.08008 & -0.4251 \\
\hline $\mathrm{H}$ & 5.902069 & 1.382518 & -0.70621 \\
\hline $\mathrm{H}$ & 5.112981 & 3.397607 & 0.280017 \\
\hline $\mathrm{H}$ & 2.672281 & 1.86465 & 1.254256 \\
\hline $\mathrm{H}$ & 2.012781 & 2.211184 & -1.14102 \\
\hline $\mathrm{H}$ & 2.257083 & 3.94286 & -0.94572 \\
\hline $\mathrm{H}$ & 0.380008 & 4.163074 & 0.618496 \\
\hline $\mathrm{H}$ & -1.39017 & 2.64612 & 0.605993 \\
\hline $\mathrm{H}$ & -1.28959 & 0.886971 & -1.22484 \\
\hline $\mathrm{H}$ & 0.452085 & 0.782989 & -1.04328 \\
\hline $\mathrm{H}$ & 0.255674 & -0.17988 & 1.194646 \\
\hline $\mathrm{H}$ & -0.57065 & -1.15678 & -0.00792 \\
\hline $\mathrm{H}$ & -1.8506 & 0.760133 & 1.998916 \\
\hline $\mathrm{H}$ & -4.00157 & 0.18468 & 1.385952 \\
\hline $\mathrm{H}$ & -3.19931 & 0.632243 & -0.106 \\
\hline
\end{tabular}




$\begin{array}{lrrr}\mathrm{H} & -3.8266 & -2.22156 & 0.81496 \\ \mathrm{H} & -5.61742 & -2.23494 & -0.77993 \\ \mathrm{H} & -5.85037 & -0.95391 & 0.40696 \\ \mathrm{H} & -4.92797 & -0.56351 & -2.5233 \\ \mathrm{H} & -6.1248 & 1.435782 & -2.53333 \\ \mathrm{H} & -6.47081 & 2.499904 & -0.24197 \\ \mathrm{H} & -7.92788 & 1.644635 & -0.73863 \\ \mathrm{H} & -6.83699 & 0.914573 & 0.451017 \\ \mathrm{H} & -1.69949 & -2.30772 & 2.01476 \\ \mathrm{H} & -1.8473 & -2.02336 & 4.423655 \\ \mathrm{H} & -2.01637 & -0.18972 & 4.219868 \\ \mathrm{H} & -2.57874 & -1.27895 & -1.83344 \\ \mathrm{H} & -1.94085 & -3.61409 & -2.05212 \\ \mathrm{H} & -2.84001 & -4.03112 & -0.48776 \\ \mathrm{H} & 2.974892 & 3.598529 & 1.417617\end{array}$

\section{Transition state TS3}

$\begin{array}{lrrr}\text { Symbol } & \mathbf{X} & \mathbf{Y} & \mathbf{Z} \\ \mathrm{C} & 5.37011 & -1.96203 & -0.40283 \\ \mathrm{C} & 6.361291 & -2.68061 & 0.148975 \\ \mathrm{C} & 7.829606 & -2.34614 & 0.186514 \\ \mathrm{C} & 5.450416 & -0.63059 & -1.1171 \\ \mathrm{C} & 4.530049 & 0.409813 & -0.52051 \\ \mathrm{C} & 4.966544 & 1.535399 & 0.110075 \\ \mathrm{C} & 4.200332 & 2.580037 & 0.744737 \\ \mathrm{C} & 2.831407 & 2.590038 & 0.92664 \\ \mathrm{C} & 1.513898 & 3.325083 & -0.95324 \\ \mathrm{C} & 0.202829 & 3.502606 & -0.45496 \\ \mathrm{C} & -0.87832 & 2.638909 & -0.43404 \\ \mathrm{C} & -0.92167 & 1.30766 & -1.13456 \\ \mathrm{C} & -0.62539 & 0.052773 & -0.26925 \\ \mathrm{C} & -1.55332 & -0.13867 & 0.956725 \\ \mathrm{C} & -3.07286 & -0.23506 & 0.635616 \\ \mathrm{C} & -3.63594 & -1.55906 & 0.067415 \\ \mathrm{C} & -5.20121 & -1.51911 & 0.05837 \\ \mathrm{C} & -5.8144 & -0.51339 & -0.88008 \\ \mathrm{C} & -6.59269 & 0.536904 & -0.57722 \\ \mathrm{C} & -7.04 & 0.999059 & 0.782927 \\ \mathrm{C} & -1.0877 & -1.30329 & 1.796271 \\ \mathrm{C} & -0.7284 & -1.23735 & 3.083275 \\ \mathrm{C} & -3.10577 & -1.92147 & -1.30001 \\ & & & 57 / 65\end{array}$




$\begin{array}{lrrr}\mathrm{C} & -2.71748 & -3.14614 & -1.67143 \\ \mathrm{H} & 4.359064 & -2.3626 & -0.31551 \\ \mathrm{H} & 6.089759 & -3.61243 & 0.648302 \\ \mathrm{H} & 8.188308 & -2.26924 & 1.222283 \\ \mathrm{H} & 8.427984 & -3.13178 & -0.2961 \\ \mathrm{H} & 8.056423 & -1.40107 & -0.31315 \\ \mathrm{H} & 6.479291 & -0.25186 & -1.11408 \\ \mathrm{H} & 5.190204 & -0.80369 & -2.17764 \\ \mathrm{H} & 3.461236 & 0.221607 & -0.61573 \\ \mathrm{H} & 6.051602 & 1.679896 & 0.139785 \\ \mathrm{H} & 4.760933 & 3.471545 & 1.026838 \\ \mathrm{H} & 2.246993 & 1.678315 & 0.853368 \\ \mathrm{H} & 1.766591 & 2.46969 & -1.5756 \\ \mathrm{H} & 2.149094 & 4.195441 & -1.08467 \\ \mathrm{H} & 0.031493 & 4.458235 & 0.052851 \\ \mathrm{H} & -1.78782 & 2.970427 & 0.066465 \\ \mathrm{H} & -1.90275 & 1.169767 & -1.61973 \\ \mathrm{H} & -0.18807 & 1.305335 & -1.95444 \\ \mathrm{H} & 0.399279 & 0.136421 & 0.112918 \\ \mathrm{H} & -0.65315 & -0.84515 & -0.90297 \\ \mathrm{H} & -1.4417 & 0.771577 & 1.560654 \\ \mathrm{H} & -3.60936 & -0.04542 & 1.575538 \\ \mathrm{H} & -3.34318 & 0.586465 & -0.03713 \\ \mathrm{H} & -2.36014 & -3.3513 & -2.67684 \\ \mathrm{H} & -3.37182 & -2.3754 & 0.753036 \\ \mathrm{H} & -5.55121 & -2.52054 & -0.23005 \\ \mathrm{H} & -5.54002 & -1.34847 & 1.086131 \\ \mathrm{H} & -5.59424 & -0.67378 & -1.93551 \\ \mathrm{H} & -6.94401 & 1.151154 & -1.4073 \\ \mathrm{H} & -6.67654 & 2.015372 & 0.983136 \\ \mathrm{H} & -8.13605 & 1.038736 & 0.84188 \\ \mathrm{H} & -6.68271 & 0.354768 & 1.589766 \\ \mathrm{H} & -0.37998 & -2.11294 & 3.625718 \\ \mathrm{H} & -3.061 & -1.11385 & -2.03101 \\ \mathrm{H} & -379228 & 1.509586\end{array}$

Ground state 11

Symbol $X$

Y

$\mathbf{Z}$ 


$\begin{array}{lrrr}\mathrm{C} & -1.65736 & -0.97848 & -0.08233 \\ \mathrm{C} & -2.67254 & -0.29895 & -0.63459 \\ \mathrm{C} & -3.12519 & 1.102696 & -0.33147 \\ \mathrm{C} & -0.70125 & -0.50681 & 0.99096 \\ \mathrm{C} & 0.731323 & -0.51278 & 0.516663 \\ \mathrm{C} & 1.550294 & 0.551876 & 0.532163 \\ \mathrm{C} & 2.939983 & 0.575769 & 0.053254 \\ \mathrm{C} & 3.444195 & -0.15148 & -0.95613 \\ \mathrm{H} & -1.47818 & -1.99307 & -0.43954 \\ \mathrm{H} & -3.25289 & -0.81302 & -1.40129 \\ \mathrm{H} & -3.09449 & 1.721784 & -1.23707 \\ \mathrm{H} & -4.16601 & 1.108187 & 0.017025 \\ \mathrm{H} & -2.51503 & 1.594453 & 0.429897 \\ \mathrm{H} & -0.96712 & 0.491234 & 1.351333 \\ \mathrm{H} & -0.7907 & -1.18779 & 1.852733 \\ \mathrm{H} & 1.110071 & -1.46555 & 0.144781 \\ \mathrm{H} & 1.179122 & 1.485551 & 0.957586 \\ \mathrm{H} & 3.601793 & 1.273029 & 0.567747 \\ \mathrm{H} & 2.823845 & -0.82146 & -1.54562 \\ \mathrm{H} & 4.492882 & -0.08445 & -1.22872\end{array}$

\section{Ground state 12}

\begin{tabular}{llrl} 
Symbol & X & \multicolumn{1}{l}{$\mathbf{Z}$} \\
C & -4.93664 & -1.09756 & -0.37818 \\
C & -3.86075 & -1.95285 & -0.12773 \\
C & -2.50424 & -1.84854 & -0.45708 \\
C & -1.94514 & -0.72605 & -1.28048 \\
C & -1.78594 & 0.657135 & -0.57503 \\
C & -0.95738 & 0.629533 & 0.732329 \\
C & 0.505395 & 0.124929 & 0.585589 \\
C & 1.564382 & 1.057512 & -0.04739 \\
C & 2.998722 & 0.45925 & 0.149068 \\
C & 3.269302 & -0.82392 & -0.591 \\
C & 3.561886 & -2.03006 & -0.08093 \\
C & 3.671242 & -2.42233 & 1.367528 \\
C & -1.02709 & 1.962048 & 1.432731 \\
C & -1.64611 & 2.195767 & 2.597985 \\
C & 1.319395 & 1.374262 & -1.50445 \\
C & 1.496633 & 2.573904 & -2.06895 \\
H & -4.82022 & -0.14076 & -0.8823 \\
H & -5.94514 & -1.37463 & -0.08196
\end{tabular}




$\begin{array}{lrrr}\mathrm{H} & -4.12254 & -2.8687 & 0.41671 \\ \mathrm{H} & -1.84032 & -2.67027 & -0.19338 \\ \mathrm{H} & -0.96886 & -1.01842 & -1.70047 \\ \mathrm{H} & -2.59648 & -0.52557 & -2.15676 \\ \mathrm{H} & -2.78636 & 1.013805 & -0.30498 \\ \mathrm{H} & -1.36909 & 1.389496 & -1.28226 \\ \mathrm{H} & -1.45667 & -0.11772 & 1.363464 \\ \mathrm{H} & 0.864543 & -0.11636 & 1.596087 \\ \mathrm{H} & 0.489104 & -0.82436 & 0.038898 \\ \mathrm{H} & 1.574746 & 2.008842 & 0.502489 \\ \mathrm{H} & 3.718393 & 1.215585 & -0.1961 \\ \mathrm{H} & 3.164062 & 0.32962 & 1.224302 \\ \mathrm{H} & 3.219734 & -0.74819 & -1.67734 \\ \mathrm{H} & 3.725219 & -2.84363 & -0.78902 \\ \mathrm{H} & 2.917015 & -3.17941 & 1.61861 \\ \mathrm{H} & 4.652038 & -2.8691 & 1.580053 \\ \mathrm{H} & 3.530318 & -1.57874 & 2.047519 \\ \mathrm{H} & -0.55852 & 2.80717 & 0.922264 \\ \mathrm{H} & -1.69034 & 3.192122 & 3.033067 \\ \mathrm{H} & -2.15286 & 1.398718 & 3.138211 \\ \mathrm{H} & 0.98979 & 0.544274 & -2.12949 \\ \mathrm{H} & 1.318947 & 2.737926 & -3.12842 \\ \mathrm{H} & 1.801493 & 3.440064 & -1.48333\end{array}$

\section{Ground state 14}

$\begin{array}{llll}\text { Symbol } & \text { X } & \text { Y } & \text { Z } \\ \text { C } & 10.55088 & 0.814462 & -0.83693 \\ \text { C } & 9.447792 & 1.664029 & -0.26332 \\ \text { C } & 8.122943 & 1.500658 & -0.40509 \\ \text { C } & 7.386806 & 0.418808 & -1.16039 \\ \text { C } & 6.489765 & -0.40389 & -0.26037 \\ \text { C } & 5.155251 & -0.49191 & -0.37709 \\ \text { C } & 4.235625 & -1.29791 & 0.488176 \\ \text { C } & 3.121644 & -0.46804 & 1.148397 \\ \text { C } & 2.079882 & -1.34655 & 1.901476 \\ \text { C } & 1.289082 & -2.29909 & 1.054841 \\ \text { C } & 0.213521 & -1.90011 & 0.257092 \\ \text { C } & -0.32443 & -0.63405 & -0.00024 \\ \text { C } & -1.58296 & -0.45203 & -0.77854 \\ \text { C } & -2.95247 & -0.36168 & 0.045688 \\ \text { C } & -2.9837 & 0.903143 & 0.842785\end{array}$




\begin{tabular}{|c|c|c|c|}
\hline $\mathrm{C}$ & -4.15182 & -0.51103 & -0.91805 \\
\hline $\mathrm{C}$ & -5.53142 & -0.8074 & -0.26747 \\
\hline $\mathrm{C}$ & -6.2433 & 0.447783 & 0.325533 \\
\hline $\mathrm{C}$ & -6.65148 & 1.495134 & -0.67872 \\
\hline $\mathrm{C}$ & -6.4654 & 2.822876 & -0.61097 \\
\hline $\mathrm{C}$ & -5.78269 & 3.608621 & 0.475486 \\
\hline $\mathrm{C}$ & -2.67428 & 1.022543 & 2.143192 \\
\hline $\mathrm{C}$ & -6.42502 & -1.534 & -1.24394 \\
\hline $\mathrm{C}$ & -6.97266 & -2.73912 & -1.04719 \\
\hline $\mathrm{H}$ & 11.19916 & 0.426279 & -0.03991 \\
\hline $\mathrm{H}$ & 10.17403 & -0.03965 & -1.40511 \\
\hline $\mathrm{H}$ & 11.19414 & 1.40327 & -1.50511 \\
\hline $\mathrm{H}$ & 9.785318 & 2.508317 & 0.339606 \\
\hline $\mathrm{H}$ & 7.471981 & 2.214555 & 0.100323 \\
\hline $\mathrm{H}$ & 6.766832 & 0.887334 & -1.93807 \\
\hline $\mathrm{H}$ & 8.100164 & -0.23218 & -1.68248 \\
\hline $\mathrm{H}$ & 6.99152 & -0.94669 & 0.543124 \\
\hline $\mathrm{H}$ & 4.67026 & 0.069345 & -1.18099 \\
\hline $\mathrm{H}$ & 3.73094 & -2.06698 & -0.11854 \\
\hline $\mathrm{H}$ & 4.81582 & -1.82918 & 1.257459 \\
\hline $\mathrm{H}$ & 3.570281 & 0.26321 & 1.840627 \\
\hline $\mathrm{H}$ & 2.595843 & 0.103663 & 0.372689 \\
\hline $\mathrm{H}$ & 1.401457 & -0.64206 & 2.421191 \\
\hline $\mathrm{H}$ & 2.618717 & -1.89548 & 2.692642 \\
\hline $\mathrm{H}$ & 1.537092 & -3.35762 & 1.099085 \\
\hline $\mathrm{H}$ & -0.29632 & -2.72395 & -0.26109 \\
\hline $\mathrm{H}$ & 0.126909 & 0.251909 & 0.443265 \\
\hline $\mathrm{H}$ & -1.55561 & 0.447005 & -1.42013 \\
\hline $\mathrm{H}$ & -1.71668 & -1.30834 & -1.45967 \\
\hline $\mathrm{H}$ & -2.94117 & -1.21933 & 0.731179 \\
\hline $\mathrm{H}$ & -3.17407 & 1.813332 & 0.267564 \\
\hline $\mathrm{H}$ & -4.2317 & 0.381861 & -1.55593 \\
\hline $\mathrm{H}$ & -3.91858 & -1.34528 & -1.59325 \\
\hline $\mathrm{H}$ & -5.35903 & -1.48578 & 0.57978 \\
\hline $\mathrm{H}$ & -5.59379 & 0.875913 & 1.092379 \\
\hline $\mathrm{H}$ & -7.14975 & 0.090394 & 0.837988 \\
\hline $\mathrm{H}$ & -7.18951 & 1.125589 & -1.55198 \\
\hline $\mathrm{H}$ & -6.85619 & 3.422226 & -1.43517 \\
\hline $\mathrm{H}$ & -5.36861 & 2.973823 & 1.261377 \\
\hline $\mathrm{H}$ & -4.96088 & 4.208675 & 0.063365 \\
\hline & -6.48327 & 4.314889 & 0.942416 \\
\hline
\end{tabular}




$\begin{array}{lrrr}\mathrm{H} & -2.61797 & 1.994444 & 2.62921 \\ \mathrm{H} & -2.43855 & 0.152722 & 2.750628 \\ \mathrm{H} & -6.60647 & -1.03362 & -2.19703 \\ \mathrm{H} & -7.59128 & -3.21668 & -1.80294 \\ \mathrm{H} & -6.81046 & -3.2935 & -0.12464\end{array}$

\section{Transition state TS4}

\begin{tabular}{lrrr} 
Symbol & X & Y & \multicolumn{1}{l}{$\mathbf{Z}$} \\
$\mathrm{C}$ & 10.89879 & 0.128197 & -0.70391 \\
$\mathrm{C}$ & 9.772354 & 1.018106 & -1.15709 \\
$\mathrm{C}$ & 8.468878 & 0.715029 & -1.25519 \\
$\mathrm{C}$ & 7.785033 & -0.59182 & -0.92634 \\
$\mathrm{C}$ & 6.751658 & -0.44023 & 0.168699 \\
$\mathrm{C}$ & 5.440573 & -0.67814 & 0.028119 \\
$\mathrm{C}$ & 4.394837 & -0.53206 & 1.095788 \\
$\mathrm{C}$ & 3.276445 & 0.459051 & 0.723489 \\
$\mathrm{C}$ & 2.148093 & 0.537336 & 1.782263 \\
$\mathrm{C}$ & 1.367414 & -0.73628 & 1.984978 \\
$\mathrm{C}$ & 0.32647 & -1.13822 & 1.193452 \\
$\mathrm{C}$ & -0.21188 & -0.50848 & 0.022843 \\
$\mathrm{C}$ & -1.34369 & -0.96708 & -0.62995 \\
$\mathrm{C}$ & -3.37161 & -0.12879 & 0.191447 \\
$\mathrm{C}$ & -3.16373 & 1.272683 & 0.138681 \\
$\mathrm{C}$ & -4.37408 & -0.81805 & -0.69318 \\
$\mathrm{C}$ & -5.74832 & -1.1617 & -0.01857 \\
$\mathrm{C}$ & -6.5488 & 0.108664 & 0.392782 \\
$\mathrm{C}$ & -7.18834 & 0.881523 & -0.73268 \\
$\mathrm{C}$ & -7.10572 & 2.197344 & -0.98782 \\
$\mathrm{C}$ & -6.32025 & 3.240057 & -0.23998 \\
$\mathrm{C}$ & -2.48938 & 2.075344 & 1.027769 \\
$\mathrm{C}$ & -6.55164 & -2.10039 & -0.87707 \\
$\mathrm{C}$ & -6.90613 & -3.35077 & -0.5509 \\
$\mathrm{H}$ & 11.42199 & 0.569401 & 0.154769 \\
$\mathrm{H}$ & 10.56035 & -0.8689 & -0.41149 \\
$\mathrm{H}$ & 11.64717 & 0.008154 & -1.49876 \\
$\mathrm{H}$ & 10.07067 & 2.029864 & -1.4349 \\
$\mathrm{H}$ & 7.793825 & 1.500949 & -1.59459 \\
$\mathrm{H}$ & 7.286699 & -0.97144 & -1.8295 \\
$\mathrm{H}$ & 8.526816 & -1.34737 & -0.63835 \\
$\mathrm{H}$ & 7.130047 & -0.09906 & 1.134312 \\
$\mathrm{H}$ & 5.074816 & -1.00722 & -0.94835
\end{tabular}




$\begin{array}{lrrr}\mathrm{H} & 3.923819 & -1.50873 & 1.279778 \\ \mathrm{H} & 4.867722 & -0.22385 & 2.039246 \\ \mathrm{H} & 3.715635 & 1.457163 & 0.576132 \\ \mathrm{H} & 2.835641 & 0.159395 & -0.23593 \\ \mathrm{H} & 1.463712 & 1.344236 & 1.479736 \\ \mathrm{H} & 2.595912 & 0.855288 & 2.736803 \\ \mathrm{H} & 1.640617 & -1.36348 & 2.832259 \\ \mathrm{H} & -0.16496 & -2.07315 & 1.478738 \\ \mathrm{H} & 0.24072 & 0.419162 & -0.32048 \\ \mathrm{H} & -1.60822 & -0.5745 & -1.60638 \\ \mathrm{H} & -1.72869 & -1.96223 & -0.41207 \\ \mathrm{H} & -3.17031 & -0.62133 & 1.144369 \\ \mathrm{H} & -3.5083 & 1.756418 & -0.78317 \\ \mathrm{H} & -4.56789 & -0.20323 & -1.58591 \\ \mathrm{H} & -3.97284 & -1.77797 & -1.06639 \\ \mathrm{H} & -5.51263 & -1.68917 & 0.916685 \\ \mathrm{H} & -5.87216 & 0.746498 & 0.968619 \\ \mathrm{H} & -7.35114 & -0.21404 & 1.074829 \\ \mathrm{H} & -7.83086 & 0.299567 & -1.39451 \\ \mathrm{H} & -7.6808 & 2.572556 & -1.83719 \\ \mathrm{H} & -5.63587 & 2.814973 & 0.496884 \\ \mathrm{H} & -5.71763 & 3.839156 & -0.93464 \\ \mathrm{H} & -6.99422 & 3.940257 & 0.274821 \\ \mathrm{H} & -2.29722 & 3.121829 & 0.806495 \\ \mathrm{H} & -2.10068 & 1.686385 & 1.965662 \\ \mathrm{H} & -6.81861 & -1.73488 & -1.87065 \\ \mathrm{H} & -7.45187 & -3.99064 & -1.2406 \\ \mathrm{H} & -6.65305 & -3.77795 & 0.417916\end{array}$

\section{Ground state 15}

\begin{tabular}{|c|c|c|c|}
\hline Symbol & $\mathbf{X}$ & $\mathbf{Y}$ & $\mathbf{Z}$ \\
\hline $\mathrm{C}$ & 6.465976 & -0.27261 & 0.278636 \\
\hline $\mathrm{C}$ & 5.499461 & 0.877578 & 0.352965 \\
\hline $\mathrm{C}$ & 4.256224 & 0.948317 & -0.14555 \\
\hline $\mathrm{C}$ & 3.497747 & -0.11491 & -0.90725 \\
\hline $\mathrm{C}$ & 2.242492 & -0.5476 & -0.18494 \\
\hline $\mathrm{C}$ & 0.99557 & -0.40085 & -0.6483 \\
\hline $\mathrm{C}$ & -0.25705 & -0.82145 & 0.072335 \\
\hline $\mathrm{C}$ & -1.19388 & 0.363209 & 0.380248 \\
\hline $\mathrm{C}$ & -2.48699 & -0.02357 & 1.136743 \\
\hline
\end{tabular}




$\begin{array}{lrrr}\mathrm{C} & -3.41195 & -0.92349 & 0.361806 \\ \mathrm{C} & -4.62326 & -0.61495 & -0.14744 \\ \mathrm{C} & -5.31745 & 0.663808 & -0.05771 \\ \mathrm{C} & -6.52842 & 0.89434 & -0.5949 \\ \mathrm{H} & 6.766594 & -0.59101 & 1.285066 \\ \mathrm{H} & 6.056031 & -1.14337 & -0.23892 \\ \mathrm{H} & 7.385027 & 0.025423 & -0.24232 \\ \mathrm{H} & 5.873069 & 1.756894 & 0.878518 \\ \mathrm{H} & 3.699318 & 1.871526 & 0.015434 \\ \mathrm{H} & 3.221552 & 0.276092 & -1.89635 \\ \mathrm{H} & 4.137858 & -0.98772 & -1.0832 \\ \mathrm{H} & 2.393765 & -1.0033 & 0.795512 \\ \mathrm{H} & 0.850786 & 0.065399 & -1.6259 \\ \mathrm{H} & -0.79524 & -1.55495 & -0.54436 \\ \mathrm{H} & 0.009542 & -1.33043 & 1.008321 \\ \mathrm{H} & -0.64355 & 1.095503 & 0.98474 \\ \mathrm{H} & -1.46426 & 0.868802 & -0.5562 \\ \mathrm{H} & -3.00289 & 0.895014 & 1.433317 \\ \mathrm{H} & -2.20215 & -0.53054 & 2.070777 \\ \mathrm{H} & -3.05431 & -1.9386 & 0.195274 \\ \mathrm{H} & -5.15831 & -1.39617 & -0.68759 \\ \mathrm{H} & -4.82306 & 1.474305 & 0.473834 \\ \mathrm{H} & -7.01499 & 1.86055 & -0.50843 \\ \mathrm{H} & -7.06561 & 0.119633 & -1.13734 \\ & & & \end{array}$

\section{Ground state 16}

\begin{tabular}{lrrr} 
Symbol & \multicolumn{1}{l}{$\mathbf{X}$} & $\mathbf{Y}$ & \multicolumn{1}{l}{$\mathbf{Z}$} \\
$\mathrm{C}$ & 0.843854 & -1.82508 & 0.063339 \\
$\mathrm{C}$ & 2.123529 & -1.42807 & -0.33363 \\
$\mathrm{C}$ & -0.41698 & -1.33982 & -0.57146 \\
$\mathrm{C}$ & -1.36733 & -0.47785 & 0.373259 \\
$\mathrm{C}$ & -0.64455 & 0.778084 & 0.931674 \\
$\mathrm{C}$ & -0.4812 & 1.940455 & -0.0144 \\
$\mathrm{C}$ & 0.653087 & 2.568747 & -0.36583 \\
$\mathrm{C}$ & 2.060921 & 2.245015 & 0.055217 \\
$\mathrm{C}$ & 3.392988 & -1.75692 & 0.146807 \\
$\mathrm{C}$ & -2.66818 & -0.16879 & -0.29937 \\
$\mathrm{C}$ & -3.85203 & -0.74829 & -0.03486 \\
$\mathrm{H}$ & 0.739893 & -2.54896 & 0.8768 \\
$\mathrm{H}$ & 2.128437 & -0.7332 & -1.18534 \\
$\mathrm{H}$ & -0.17746 & -0.72892 & -1.45639
\end{tabular}




$\begin{array}{lrrr}\mathrm{H} & -1.06561 & -2.16132 & -0.93206 \\ \mathrm{H} & -1.57893 & -1.12008 & 1.24047 \\ \mathrm{H} & 0.32898 & 0.43 & 1.293712 \\ \mathrm{H} & -1.21549 & 1.13751 & 1.802706 \\ \mathrm{H} & -1.40579 & 2.344757 & -0.42956 \\ \mathrm{H} & 0.554862 & 3.425234 & -1.03753 \\ \mathrm{H} & 2.150775 & 1.277497 & 0.553362 \\ \mathrm{H} & 2.72549 & 2.216872 & -0.81768 \\ \mathrm{H} & 2.457111 & 3.022158 & 0.725858 \\ \mathrm{H} & 4.288182 & -1.381 & -0.34096 \\ \mathrm{H} & 3.533272 & -2.43977 & 0.984038 \\ \mathrm{H} & -2.62199 & 0.52811 & -1.1389 \\ \mathrm{H} & -4.74298 & -0.52759 & -0.61914 \\ \mathrm{H} & -3.96342 & -1.4661 & 0.776214\end{array}$

